\title{
Applications, phytochemistry, pharmacological effects, pharmacokinetics, toxicity of Scutellaria baicalensis Georgi. and its probably potential therapeutic effects on COVID-19: a review
}

\author{
Jia-Wen Song, Jia-Ying Long, Long Xie, Lin-Lin Zhang, Qing-Xuan Xie, Hui-Juan Chen, Mao Deng \\ and Xiao-Fang Li*
}

\begin{abstract}
Scutellaria baicalensis Georgi. (SB) is a common heat-clearing medicine in traditional Chinese medicine (TCM). It has been used for thousands of years in China and its neighboring countries. Clinically, it is mostly used to treat diseases such as cold and cough. SB has different harvesting periods and processed products for different clinical symptoms. Botanical researches proved that SB included in the Chinese Pharmacopoeia (1st, 2020) was consistent with the medicinal SB described in ancient books. Modern phytochemical analysis had found that SB contains hundreds of active ingredients, of which flavonoids are its major components. These chemical components are the material basis for SB to exert pharmacological effects. Pharmacological studies had shown that SB has a wide range of pharmacological activities such as antiinflammatory, antibacterial, antiviral, anticancer, liver protection, etc. The active ingredients of SB were mostly distributed in liver and kidney, and couldn't be absorbed into brain via oral absorption. SB's toxicity was mostly manifested in liver fibrosis and allergic reactions, mainly caused by baicalin. The non-medicinal application prospects of SB were broad, such as antibacterial plastics, UV-resistant silk, animal feed, etc. In response to the Coronavirus Disease In 2019 (COVID-19), based on the network pharmacology research, SB's active ingredients may have potential therapeutic effects, such as baicalin and baicalein. Therefore, the exact therapeutic effects are still need to be determined in clinical trials. SB has been reviewed in the past 2 years, but the content of these articles were not comprehensive and accurate. In view of the above, we made a comprehensive overview of the research progress of SB, and expect to provide ideas for the follow-up study of SB.
\end{abstract}

Keywords: SB applications, Phytochemistry, Pharmacological effects, Pharmacokinetics, Toxicity, Treating COVID-19

\section{Background}

$\mathrm{SB}$, a species in the genus Scutellaria (family Lamiaceae) which its dried root is a TCM recorded in ChP (1st, 2020) [1], often been called Huangqin or Skullcap.

*Correspondence: lixiaofang@cdutcm.edu.cn

School of Pharmacy, Chengdu University of Traditional Chinese Medicine, No. 1166, Liutai Avenue, Chengdu 611137, China
SB has been widely used for thousands of years by China and its neighbors. It mainly grows in temperate regions and tropical mountains (with an altitude of about 1300$3000 \mathrm{~m}$ ), including China, Russia's Eastern Siberia, Mongolia, North Korea, Japan, etc. [2]. The traditional therapeutic effects have been first recorded in the Shennong Bencaojing (The Classic of Herbal Medicine) [3], an existing original pharmacological monograph of China

(c) The Author(s) 2020. This article is licensed under a Creative Commons Attribution 4.0 International License, which permits use, sharing, adaptation, distribution and reproduction in any medium or format, as long as you give appropriate credit to the original author(s) and the source, provide a link to the Creative Commons licence, and indicate if changes were made. The images or other third party material in this article are included in the article's Creative Commons licence, unless indicated otherwise in a credit line to the material. If material is not included in the article's Creative Commons licence and your intended use is not permitted by statutory regulation or exceeds the permitted use, you will need to obtain permission directly from the copyright holder. To view a copy of this licence, visit http://creativeco mmons.org/licenses/by/4.0/. The Creative Commons Public Domain Dedication waiver (http://creativecommons.org/publicdomain/ zero/1.0/) applies to the data made available in this article, unless otherwise stated in a credit line to the data. 
written by many medical scientists together during the Han dynasty. It narrated in detail that SB has "the effects of clearing away the pathology of heat and dampness, eliminating fire pathology, detoxifying, and preventing bleeding and fetal restlessness" [1].

SB contains various chemical components, such as various flavonoids, diterpenes, polyphenols, amino acids, volatile oils, sterols, benzoic acids, etc. $[4,5]$. Flavonoids are the main components of SB. There are more than 110 kinds of flavonoids in its dried roots, including baicalin, baicalein, wogonoside, wogonin, etc. These are the main material basis for SB to play clinical effects. SB has antibacterial, antiviral, anti-inflammatory, anticancer, liver and nerve protective functions, etc. [6-8]. Because SB has a very wide range of pharmacological effects and clinical applications, more and more in-depth researches had been implemented. This paper is a comprehensive summary of the current researches on SB, including clinical and non-clinical applications, medicinal materials, botany, phytochemistry, pharmacology, pharmacokinetic characteristics, and toxicity. And then, we also discussed that SB has a certain potential effects in the treatment of the current major disease-COVID-19 based on the current network pharmacology researches. Its possible prevention and treatment mechanisms were discussed, but the exact therapeutic effects still need to be determined in the next step.

\section{Applications \\ Clinical applications}

SB is one of the 40 bulk medicinal materials and one of the famous "three yellow" in TCM [9]. It is a common medicine for treating cold, flu, fever, diarrhea, jaundice, headache, abdominal pain, drenching, etc. SB is frequently used in classical prescription, such as Mahuang Shengma decoction, Xiexin decoction and Huangqin decoction, ect [10]. In modern clinical applications, the quantity or dosage of TCM in prescriptions can be added or reduced to clearing away heat with different symptoms [11]. Compared with other antipyretic TCM, SB is more suitable for heat syndrome with fetal restlessness. There are about 477 prescriptions containing SB [12] for treating 153 main diseases, cough and cold are more frequently used. And after searching ChP. (4th, 2020) [13], for the treatment of cold, more than $30 \%$ of Chinese patent medicine contained SB active ingredients, and for clearing away heat and detoxifying more than $70 \%$ of them contained SB and its active ingredients. Common forms of these preparations include granule, pill, tablet, liquid preparation, capsule, etc. [14]. The same prescription may have different preparation forms, and the appropriate form should be selected according to the development of the disease.

\section{Non-clinical applications}

A composite material contained SB had good biocompatibility and strong antibacterial activity [15]. Many components (e.g. baicalin, baicalein and wogonin) had reversible electronic shuttle activity for the extraction of bioenergy [16]. Microbial fuel cells contained baicalin and baicalein had higher ability of bioelectricity generation, and showed weaker biological toxicity during the process of generating electricity. Feed contained SB flavonoids improved the meat quality and antioxidant capacity of broilers and increased the activity of SOD, GSH and peroxidase in liver [17]. It is suggested that SB flavonoids can be added into agricultural feed to improve meat quality. A silk product contained baicalin was successfully prepared [18], and it had antibacterial, anti-oxidation and UV protection effects. In addition, it also showed an electrostatic effect. SBE had the effect of inhibiting the synthesis of melanin, and had good safety to the skin [19]. It can be used as a whitening ingredient in the beauty industry after more clinical trials based on human skin safety.

\section{Botanical researches}

\section{Textual researches of botany}

Genuine SB is the dried root of perennial herb SB of the Labiaceae family. It is widely distributed in Hebei, Shandong, Shanxi and other regions in China. It is suitable for growing in the dry sunny places such as the mountain top, hillside, forest edge and roadside with an altitude of 500-1500 m [20]. Illustrated Pharmacopoeia (Ben Cao Tu Jing) narrated that "The seedling is more than footlong, the stem is as thick as chopsticks, and the leaves are clustered from all sides of the ground". Compendium of Materia Medica (Ben Cao Gang Mu) also narrated that "Kuqin (rotten xylem) is an old root with hollow and yellow outside and black inside. Ziqin (strip types) is a new root with more compact inside. SB in southwest is hollow and black, contrast to SB in north is solid and deep yellow". The former is mainly used to treat lung heat cough and the latter is mainly used to treat damp heat dysentery. However, there is no excessive differentiation in clinical use [21]. Therefore, the plant morphology of SB described by the ancients is consistent with that of $\mathrm{SB}$ used now [22].

\section{Harvesting and processing}

In addition to the regulation of genetic factors and the influence of environmental conditions [23], the quality of effective components of TCM is also affected by harvesting and processing [24]. Studies have shown that there are differences in the content of SB in spring and autumn [25]. The best harvest time of SB is determined 
by the highest content of baicalin, wogonoside, baicalein and scutellarin, most of which are concentrated in September [26]. According to the growth condition of $\mathrm{SB}$ and the dynamic accumulation of baicalin, there was a significant increase of baicalin in triennial compared with biennials in the root [20]. Therefore, the harvest time of SB is mainly concentrated in autumn, and the quality of triennial one is the best.

The traditional processing of SB needs to bump away the rough skin. However, the key enzymes for the synthesis of flavonoids were found to be mainly concentrated in the phloem of the root according to spatial imaging [27]. The content of baicalin in cortex is the highest, which affects the quality of SB. Therefore, it is suggested that the above-mentioned step should be cancelled in the modern processing of $\mathrm{SB}$, so as to ensure the quality of SB $[28,29]$. The total amount of baicalin and flavonoids in the process of drying shows a change trend of inverted "V", which may be related to the physiological mechanism of anti-drought stress of SB root itself $[26,30]$. This showed that the drying time should not be too long, otherwise the content of active ingredients will be reduced. At present, there are different processed products of $\mathrm{SB}$, such as wine, fried and charcoal SB respectively. Different processed products of SB play different roles in the prescription [31]. The commonly used processed products of SB are raw materials, which can play the role of clearing away heat and detoxification. Wine SB can play the role of tonifying and moistening in addition to clearing away heat.

\section{Cultivation conditions}

By controlling the cultivation conditions, the content of the effective components of planted SB can be improved. Yuan et al. found that the lack of water can cause the hormone metabolism in SB and affect the synthesis of baicalin and other flavonoids [32]. The synthesis of different flavonoids in SB will change differently under UV-B irradiation [33]. For example, the content of chrysin, scutellarin, baicalin and tectoridin would changed under different intensity UV-B irradiation. Free salicylic acid has a great effect on the accumulation of baicalin in the growth of SB, but it has little effect on baicalein [34]. Blue, white and red light has different effects on the content of flavonoids [35]. The content of baicalin increased with the extension of illumination time, and its content reached the maximum under blue light. These studies indicated that the content of water, free salicylic acid, UV-B irradiation, and different light time and intensity could be regulated to change the content of flavonoids in the cultivation process of SB.

\section{Phytochemistry}

In 1970s, many chemical components of SB had been separated one after another. These components are the material basis for SB to play a wide range of pharmacological actions [36]. There are hundreds of ingredients in SB (shown in Table 1 and Fig. 1), in addition to flavonoids, it also contains volatile oils, terpenoids, polysaccharides, phenylethyl, amino acids, sterols, starch, alkaloids, organic acids and trace elements [40]. Flavonoids are the major components of SB. There are more than 100 kinds of flavonoids in SB, it is important to note that pharmacological activities of SB take place due to the presence of specific 4'-deoxyflavones such as chrysin, baicalein, wogonin and their glycosides (baicalin and wogonoside) [41]. The key enzymes for the synthesis of these compounds are mainly found in roots, including PAL, C4H, CLL, CHS, CHI, FNSII, MT, GT, FH and OMT, different flavonoid synthetases are encoded by different genes $[6,42,43]$. The main synthetic routes of flavonoids were shown in Fig. 2.

Volatile oils, as secondary metabolites of plants, are widely distributed in the whole plant. Acetophenone, 1-phenyl-1,3-butanediol, palmitic acid and oleic acid are the most abundant [44]. The content and composition of volatile oils in different parts are different [45]. For example, germacrene $\mathrm{D}$, bornyl acetate, diphenylamine and hexadecanoic acid are the primary oils in flowers, stem leaves, roots and seeds respectively. Terpenoids are mainly distributed in the aboveground part and not exist in seeds [46]. They have antibacterial, antiviral, antipyretic, analgesic and anti-inflammatory effects [47].

The polysaccharides of SB are water-soluble, mainly composed of arabinose, galactose, glucose and some differential branched dextran [48-50]. At present, a group of new polysaccharides SP1-1 with molecular weight of $4.56 \times 10^{5} \mathrm{Da}$ was identified and had anti-inflammatory effect [51], which is mainly composed of mannose, ribose, glucuronic acid, glucose, xylose and arabinose (the molar ratio were 2.14:3.61:1:2.86:5.98:36.39).

There are 14 kinds of amino acids in SB, of which proline is the highest, accounting for $80 \%$ [52]. Sterols contain $\alpha$-spinasterol and $\beta$-sitosterol [40], among them, $\beta$-sitosterol has been proved to be effective for anorexia. Phenolic compounds, as organic acid components of medicinal plants, such as citric acid, tartaric acid, malonic acid, etc., have influence on the growth of plants [53]. Exception for lutein and $\beta$-carotene [54], there also are phytoene, zeaxanthin and $\xi$-carotene. And there are 3 lignin glycosides in SB [55]. Platinum is is mainly concentrated in roots, which its content related to the growth of SB [56]. Recently, a new actinomycete isolated from the cortex of SB [57], named Brachybacterium endophyticum sp. nov., was identified as a gram-positive, aerobic, 
Table 1 The components of SB

\begin{tabular}{|c|c|c|c|c|c|}
\hline No. & Name & Molecular formula & Weight & Plant part & Ref. \\
\hline 1 & Baicalein (5,6,7-Trihydroxyflavone) & $\mathrm{C}_{15} \mathrm{H}_{10} \mathrm{O}_{5}$ & 270 & $\begin{array}{l}\text { Root } \\
\text { Hairy Root }\end{array}$ & [37] \\
\hline 2 & Methoxybaicalein (5,6-Dihydroxy-7-methoxyflavone) & $\mathrm{C}_{16} \mathrm{H}_{12} \mathrm{O}_{5}$ & 284 & Root & [117] \\
\hline 3 & Scutellarein (5,6,7,4'-Tetrahydroxyflavone) & $\mathrm{C}_{15} \mathrm{H}_{10} \mathrm{O}_{6}$ & 286 & Root & [39] \\
\hline 4 & 5,6,7-Trihydroxy-4'-methoxyflavone & $\mathrm{C}_{16} \mathrm{H}_{12} \mathrm{O}_{6}$ & 300 & Aerial part & [38] \\
\hline 5 & Oroxylin A (5,7-Dihydroxy-6-methoxyflavone) & $\mathrm{C}_{16} \mathrm{H}_{12} \mathrm{O}_{5}$ & 284 & Root & [37] \\
\hline 6 & Tenaxin II (5,7,2'-Trihydroxy-6-methoxyflavone) & $\mathrm{C}_{16} \mathrm{H}_{12} \mathrm{O}_{6}$ & 300 & Root & [38] \\
\hline 7 & 5,7,4'-Trihydroxy-6-methoxyflavone & $\mathrm{C}_{16} \mathrm{H}_{12} \mathrm{O}_{6}$ & 300 & Aerial part & [38] \\
\hline 8 & 5,7-Dihydroxy-6,8-dimethoxyflavone & $\mathrm{C}_{17} \mathrm{H}_{14} \mathrm{O}_{6}$ & 314 & Root & [38] \\
\hline 9 & 5,7,2'-Trihydroxy-6,8-dimethoxyflavone & $\mathrm{C}_{17} \mathrm{H}_{14} \mathrm{O}_{7}$ & 330 & Root & [38] \\
\hline 10 & 5,8-Dihydroxy-6,7-dimethoxyflavone & $\mathrm{C}_{17} \mathrm{H}_{14} \mathrm{O}_{6}$ & 314 & Root & [38] \\
\hline 11 & 5,8,2'-Trihydroxy-6,7-dimethoxyflavone & $\mathrm{C}_{17} \mathrm{H}_{14} \mathrm{O}_{7}$ & 330 & Root & [38] \\
\hline 12 & Tenaxin I (5,2'-Dihydroxy-6,7,8-trimethoxyflavdne) & $\mathrm{C}_{18} \mathrm{H}_{16} \mathrm{O}_{7}$ & 344 & Root & [38] \\
\hline 13 & 5,2',5'-Trihydroxy-6,7,8-trimethoxyflavone & $\mathrm{C}_{18} \mathrm{H}_{16} \mathrm{O}_{8}$ & 360 & Root & [38] \\
\hline 14 & Skullcapflavone II (5,6'-Dihydroxy-6,7,8,2'-tetramethoxyflavone) & $\mathrm{C}_{19} \mathrm{H}_{18} \mathrm{O}_{8}$ & 374 & $\begin{array}{l}\text { Root } \\
\text { Hairy Root }\end{array}$ & [37] \\
\hline 15 & 5,4'-Dihydroxy-6,7,3',5'-tetramethoxyflavone & $\mathrm{C}_{19} \mathrm{H}_{18} \mathrm{O}_{8}$ & 374 & Aerial part & [38] \\
\hline 16 & 5,2'-Dihydroxy-6,7,8,3'-tetramethoxyflavone & $\mathrm{C}_{19} \mathrm{H}_{18} \mathrm{O}_{8}$ & 374 & Hairy Root & [38] \\
\hline 17 & Chrysin (5,7-Dihydroxyflavone) & $\mathrm{C}_{15} \mathrm{H}_{10} \mathrm{O}_{4}$ & 254 & $\begin{array}{l}\text { Root } \\
\text { Aerial part }\end{array}$ & [37] \\
\hline 18 & Norwogonin (5,7,8-Trihydroxyflavone) & $\mathrm{C}_{15} \mathrm{H}_{10} \mathrm{O}_{5}$ & 270 & Root & [38] \\
\hline 19 & Isoscutellarein (5,7,8,4'-Tetrahydroxyflavone) & $\mathrm{C}_{15} \mathrm{H}_{10} \mathrm{O}_{6}$ & 286 & Aerial part & [38] \\
\hline 20 & Apigenin (5,7,4'-Trihydroxyflavone) & $\mathrm{C}_{15} \mathrm{H}_{10} \mathrm{O}_{5}$ & 270 & $\begin{array}{l}\text { Root } \\
\text { Aerial part }\end{array}$ & [39] \\
\hline 21 & 4'-Hydroxywogonin (5,7 4'-Trihydroxy-8-methoxyflavone) & $\mathrm{C}_{16} \mathrm{H}_{12} \mathrm{O}_{6}$ & 300 & Root & [38] \\
\hline 22 & 2'-Hydroxychrysin (5,7,2'-Trihydroxyflavone) & $\mathrm{C}_{15} \mathrm{H}_{10} \mathrm{O}_{5}$ & 270 & Root & [38] \\
\hline 23 & $5,7,2^{\prime}, 3^{\prime}$-Tetrahydroxyflavone & $\mathrm{C}_{15} \mathrm{H}_{10} \mathrm{O}_{6}$ & 286 & Root & {$[38]$} \\
\hline 24 & 6-Hydroxyluteolin & $\mathrm{C}_{15} \mathrm{H}_{10} \mathrm{O}_{7}$ & 292 & Whole plant & [39] \\
\hline 25 & Salvigenin & $\mathrm{C}_{18} \mathrm{H}_{16} \mathrm{O}_{7}$ & 344 & Root & {$[38]$} \\
\hline 27 & Luteolin & $\mathrm{C}_{15} \mathrm{H}_{10} \mathrm{O}_{6}$ & 286 & Whole plant & {$[39]$} \\
\hline 26 & $5,7,2^{\prime}, 5^{\prime}$-Tetrahydroxyflavone & $\mathrm{C}_{15} \mathrm{H}_{10} \mathrm{O}_{6}$ & 286 & Root & [38] \\
\hline 28 & $5,7,2^{\prime}, 6^{\prime}$-Tetrahydroxyflavone & $\mathrm{C}_{15} \mathrm{H}_{10} \mathrm{O}_{6}$ & 286 & Root & [38] \\
\hline 29 & 5,7,6'-Trihydroxy-2'-methoxyflavone & $\mathrm{C}_{16} \mathrm{H}_{12} \mathrm{O}_{6}$ & 300 & Root & [38] \\
\hline 30 & 5,7-Dihydroxy-6,8,2',3'-tertramethoxyflavone & $\mathrm{C}_{19} \mathrm{H}_{18} \mathrm{O}_{7}$ & 358 & Root & [38] \\
\hline 31 & Wogonin (5,7-Dihydroxy-8-methoxyflavone) & $\mathrm{C}_{16} \mathrm{H}_{12} \mathrm{O}_{5}$ & 284 & $\begin{array}{l}\text { Root } \\
\text { Aerial part } \\
\text { Hairy Root }\end{array}$ & [37] \\
\hline 32 & 3,5,4'-Trihydroxy-6,7,8-trimethoxyflavone & $\mathrm{C}_{18} \mathrm{H}_{16} \mathrm{O}_{8}$ & 360 & Whole plant & [39] \\
\hline 33 & Scutevulin (5,7,2'-Trihydroxy-8-methoxyflavone) & $\mathrm{C}_{16} \mathrm{H}_{12} \mathrm{O}_{6}$ & 300 & Root & {$[38]$} \\
\hline 34 & 5,7,6'-Trihydroxy-8,2'-dimethoxyflavone & $\mathrm{C}_{17} \mathrm{H}_{14} \mathrm{O}_{7}$ & 330 & Root & [37] \\
\hline 35 & Viscidulin III (5,7,3', 6'-Tetrahydroxy-8,2'-dimethoxyflavone) & $\mathrm{C}_{17} \mathrm{H}_{14} \mathrm{O}_{8}$ & 346 & Root & [38] \\
\hline 36 & $5,7,2^{\prime}$-Trihydroxy-6'-methoxyflavone & $\mathrm{C}_{16} \mathrm{H}_{12} \mathrm{O}_{6}$ & 300 & Root & [38] \\
\hline 37 & 5,7-Dihydroxy-8, 2', 3', 6'-tetramethoxyflavone & $\mathrm{C}_{19} \mathrm{H}_{18} \mathrm{O}_{8}$ & 374 & Root & [38] \\
\hline 38 & 7-Methoxychrysin (5-Hydroxy-7-methoxyflavone) & $\mathrm{C}_{16} \mathrm{H}_{12} \mathrm{O}_{4}$ & 268 & Aerial part & [38] \\
\hline 39 & 5,8-Dihydroxy-7-methoxyflavone & $\mathrm{C}_{16} \mathrm{H}_{12} \mathrm{O}_{5}$ & 284 & Root & [38] \\
\hline 40 & Genkwanin (5.,4'-Dihydroxy-7-methoxyflavone) & $\mathrm{C}_{16} \mathrm{H}_{12} \mathrm{O}_{5}$ & 284 & Aerial part & [38] \\
\hline 41 & 5,8,2'-Trihydroxy-7-methoxyflavone & $\mathrm{C}_{16} \mathrm{H}_{12} \mathrm{O}_{6}$ & 300 & Root & [38] \\
\hline 42 & 7-O-Methylwogonin (5-Hydroxy-7,8-dimethoxyflavone) & $\mathrm{C}_{17} \mathrm{H}_{14} \mathrm{O}_{5}$ & 298 & Root & [38] \\
\hline 43 & 5,7,4'-Trihydroxy-8-methoxyflavone & $\mathrm{C}_{17} \mathrm{H}_{12} \mathrm{O}_{6}$ & 312 & Root & [38] \\
\hline
\end{tabular}


Table 1 (continued)

\begin{tabular}{|c|c|c|c|c|c|}
\hline No. & Name & Molecular formula & Weight & Plant part & Ref. \\
\hline 44 & Skullcapflavone I (5,2'-Dihydroxy-7,8-dimethoxyflavone) & $\mathrm{C}_{17} \mathrm{H}_{14} \mathrm{O}_{6}$ & 314 & $\begin{array}{l}\text { Root } \\
\text { Hairy Root }\end{array}$ & [38] \\
\hline$\overline{45}$ & Viscidulin II (5,2',6'-Trihydroxy-7,8-dimethoxyflavone) & $\mathrm{C}_{17} \mathrm{H}_{14} \mathrm{O}_{7}$ & 330 & Root & [38] \\
\hline 46 & Rivularin (5,6'-Dihydroxy-7,8,2'-trimethoxyflavone) & $\mathrm{C}_{18} \mathrm{H}_{16} \mathrm{O}_{7}$ & 344 & $\begin{array}{l}\text { Root } \\
\text { Hairy Root }\end{array}$ & [38] \\
\hline 47 & 6'-Hydroxy-5,6,7,8,2'-pentamethoxyflavone & $\mathrm{C}_{20} \mathrm{H}_{20} \mathrm{O}_{8}$ & 388 & Root & [38] \\
\hline 48 & 6, $\sigma^{\prime}$-Dihydroxy-5,7,8,2'-tetramethoxyflavone & $\mathrm{C}_{19} \mathrm{H}_{18} \mathrm{O}_{8}$ & 374 & Root & [38] \\
\hline 49 & $5,7,3^{\prime} 4^{\prime}, 5^{\prime}-$ Pentamethoxyflavone & $\mathrm{C}_{20} \mathrm{H}_{20} \mathrm{O}_{7}$ & 372 & Aerial part & [38] \\
\hline 50 & Viscidulin I (5,7,2',6'-Tetrahydroxyflavonol) & $\mathrm{C}_{15} \mathrm{H}_{10} \mathrm{O}_{7}$ & 302 & Root & [37] \\
\hline 51 & 5,7,6'-Trihydroxy-2'-methoxyflavonol & $\mathrm{C}_{16} \mathrm{H}_{12} \mathrm{O}_{7}$ & 316 & Root & [38] \\
\hline 52 & Baicalein 6-O-glucuronide & $\mathrm{C}_{21} \mathrm{H}_{18} \mathrm{O}_{11}$ & 446 & Whole plant & [39] \\
\hline 53 & 6-Hydroxyluteolin 7-O-glucoronide & $\mathrm{C}_{21} \mathrm{H}_{18} \mathrm{O}_{13}$ & 478 & Whole plant & [39] \\
\hline 54 & Luteolin 7-O-glucuronide & $\mathrm{C}_{21} \mathrm{H}_{18} \mathrm{O}_{12}$ & 462 & Whole plant & [39] \\
\hline 55 & Apigenin 7,4'-di-O-rhamnoside & $\mathrm{C}_{21} \mathrm{H}_{20} \mathrm{O}_{8}$ & 400 & Whole plant & [39] \\
\hline 56 & 8-Methoxy-5-O-glucosideflavone & $\mathrm{C}_{22} \mathrm{H}_{20} \mathrm{O}_{10}$ & 444 & Root & [38] \\
\hline 57 & Apigenin 7-O- $\beta$-D-glucoside & $\mathrm{C}_{21} \mathrm{H}_{20} \mathrm{O}_{10}$ & 432 & Aerial part & [39] \\
\hline 58 & Baicalein 7-O- $\beta$-D-glucoside & $\mathrm{C}_{21} \mathrm{H}_{20} \mathrm{O}_{10}$ & 432 & $\begin{array}{l}\text { Root } \\
\text { Aerial part }\end{array}$ & [37] \\
\hline 59 & Oroxylin A 7-O- $\beta$-D-glucoside & $\mathrm{C}_{21} \mathrm{H}_{20} \mathrm{O}_{10} \mathrm{O}$ & 446 & $\begin{array}{l}\text { Aerial part } \\
\text { Root }\end{array}$ & {$[37]$} \\
\hline 60 & Apigenin 6-C-glucosyl-8-C-arabinoside & $\mathrm{C}_{26} \mathrm{H}_{28} \mathrm{O}_{14}$ & 564 & Whole plant & [39] \\
\hline 61 & 5,6'-Dihydroxy-7,8-dimethoxyflavone 2'-O- $\beta$-D-glucoside & $\mathrm{C}_{23} \mathrm{H}_{24} \mathrm{O}_{12}$ & 492 & $\begin{array}{l}\text { Root } \\
\text { Hairy Root }\end{array}$ & [37] \\
\hline 62 & 5,6'-Dihydroxy-6,7,8-trimethoxyflavone 2'-O- $\beta$-D-glucoside & $\mathrm{C}_{24} \mathrm{H}_{26} \mathrm{O}_{13}$ & 522 & Root & [38] \\
\hline 63 & 5,6'-Dihydroxy-6,7-dimethoxyflavone 2'-O- $\beta$-D-glucoside & $\mathrm{C}_{23} \mathrm{H}_{24} \mathrm{O}_{12}$ & 492 & $\begin{array}{l}\text { Root } \\
\text { Hairy Root }\end{array}$ & $\begin{array}{l}{[38]} \\
{[38]}\end{array}$ \\
\hline 64 & $5,7,6^{\prime}$-Trihydroxyflavone 2'-O- $\beta$-D-glucoside & $\mathrm{C}_{21} \mathrm{H}_{20} \mathrm{O}_{11}$ & 448 & Hairy Root & [37] \\
\hline 65 & Viscidulin III 6'-O- $\beta$-D-glucoside & $\mathrm{C}_{23} \mathrm{H}_{24} \mathrm{O}_{13}$ & 508 & $\begin{array}{l}\text { Root } \\
\text { Hairy Root }\end{array}$ & [37] \\
\hline 66 & Wogonin 5-O- $\beta$-D-glucoside & $\mathrm{C}_{22} \mathrm{H}_{22} \mathrm{O}_{10}$ & 446 & Root & [37] \\
\hline 67 & 3,5,7,6'-Tetrahydroxyflavone 2'-O- $\beta$-D-glucoside & $\mathrm{C}_{21} \mathrm{H}_{20} \mathrm{O}_{12}$ & 464 & Root & [38] \\
\hline 68 & Kaempferol 3-O- $\beta$-D-glucoside & $\mathrm{C}_{21} \mathrm{H}_{20} \mathrm{O}_{11}$ & 448 & Aerial part & [38] \\
\hline 69 & Chrysin 7-O- $\beta$-D-glucuronide & $\mathrm{C}_{21} \mathrm{H}_{18} \mathrm{O}_{10}$ & 430 & $\begin{array}{l}\text { Root } \\
\text { Aerial part }\end{array}$ & [37] \\
\hline 70 & Baicalin (5,6-Dihydroxyflavone 7-O- $\beta$-D-glucuronide) & $\mathrm{C}_{21} \mathrm{H}_{18} \mathrm{O}_{11}$ & 446 & $\begin{array}{l}\text { Root } \\
\text { Aerial part } \\
\text { Hairy Root }\end{array}$ & [37] \\
\hline 71 & 5,2'-Dihydroxy-6-methoxyflavone 7-O- $\beta$-D-glucuronide & $\mathrm{C}_{22} \mathrm{H}_{20} \mathrm{O}_{12}$ & 476 & Root & [37] \\
\hline 72 & Wogonoside (Wogonin 7-O- $\beta$-D-glucuronide) & $\mathrm{C}_{22} \mathrm{H}_{20} \mathrm{O}_{11}$ & 460 & $\begin{array}{l}\text { Root } \\
\text { Hairy Root }\end{array}$ & [37] \\
\hline 73 & Oroxyloside (Oroxylin A 7-O- $\beta$-D-glucuronide) & $\mathrm{C}_{22} \mathrm{H}_{20} \mathrm{O}_{11}$ & 460 & Root & [37] \\
\hline 74 & Norwogonin 7-O- $\beta$-D-glucuronide (5,8-dihydroxyflavone 7-O- $\beta$-D-glucuronide) & $\mathrm{C}_{21} \mathrm{H}_{18} \mathrm{O}_{11}$ & 446 & Root & [37] \\
\hline 75 & Isoscutellarein 8-O- $\beta$-D-glucuronide & $\mathrm{C}_{24} \mathrm{H}_{24} \mathrm{O}_{12}$ & 504 & Leaf & [38] \\
\hline 76 & 5-Hydroxy-7,8,6'-trimethoxyflavone 2'-O- $\beta$-D-glucuronide & $\mathrm{C}_{24} \mathrm{H}_{24} \mathrm{O}_{13}$ & 520 & Hairy Root & [38] \\
\hline 77 & Scutellarin & $\mathrm{C}_{21} \mathrm{H}_{18} \mathrm{O}_{12}$ & 462 & Root & [37] \\
\hline 78 & Apigenin 7-O- $\beta$-D-glucuronide & $\mathrm{C}_{21} \mathrm{H}_{18} \mathrm{O}_{11}$ & 446 & Aerial part & [39] \\
\hline 79 & $\begin{array}{l}\text { Patuletin 7-O- } \beta \text {-D-glucuronide (3,5,3',4'-Tetrahydroxy-6-methoxyflavone 7-O- } \beta \text {-D- } \\
\text { glucuronide) }\end{array}$ & $\mathrm{C}_{22} \mathrm{H}_{20} \mathrm{O}_{14}$ & 508 & Root & [38] \\
\hline 80 & Chrysin 8-C- $\beta$-D-glucoside & $\mathrm{C}_{21} \mathrm{H}_{20} \mathrm{O}_{9}$ & 416 & Root & [37] \\
\hline 81 & Chrysin 6-C- $\beta$-D-glucoside & $\mathrm{C}_{21} \mathrm{H}_{20} \mathrm{O}_{9}$ & 416 & Root & [38] \\
\hline 82 & Chrysin 6-C- $\beta-D-$ glucoside-8-C-a-L-arabinopyranoside & $\mathrm{C}_{26} \mathrm{H}_{28} \mathrm{O}_{13}$ & 548 & $\begin{array}{l}\text { Root } \\
\text { Hairy Root }\end{array}$ & [37] \\
\hline
\end{tabular}


Table 1 (continued)

\begin{tabular}{|c|c|c|c|c|c|}
\hline No. & Name & Molecular formula & Weight & Plant part & Ref. \\
\hline 83 & Chrysin 6-C-a-L-arabinopyranoside-8-C- $\beta$-D-glucoside & $\mathrm{C}_{26} \mathrm{H}_{2} 8 \mathrm{O}_{13}$ & 548 & $\begin{array}{l}\text { Root } \\
\text { Hairy Root }\end{array}$ & {$[37]$} \\
\hline 84 & Chrysin 6-C- $\beta$-L-arabinopyranoside-8-C- $\beta$-D-glucoside & $\mathrm{C}_{26} \mathrm{H}_{28} \mathrm{O}_{13}$ & 548 & Root & [39] \\
\hline 85 & Chrysin 6-C- $\beta$-D-glucoside-8-C- $\beta$-L-arabinopyranoside & $\mathrm{C}_{26} \mathrm{H}_{28} \mathrm{O}_{13}$ & 548 & Root & [39] \\
\hline 86 & Chrysin 6-C- $\beta$-arabinofuranoside-8-C- $\beta$-D-glucoside & $\mathrm{C}_{26} \mathrm{H}_{28} \mathrm{O}_{13}$ & 548 & Root & [39] \\
\hline 87 & Chrysin 6-C- $\beta$-D-glucoside-8-C- $\beta$-arabinofuranoside & $\mathrm{C}_{26} \mathrm{H}_{28} \mathrm{O}_{13}$ & 548 & Root & [39] \\
\hline 88 & Chrysin 3-C-a-arabinopyranoside-8-C- $\beta$-D-glucoside & $\mathrm{C}_{26} \mathrm{H}_{28} \mathrm{O}_{13}$ & 548 & Root & [39] \\
\hline 89 & Apigenin 6-C-a-L-arabinopyranoside-8-C- $\beta$-D-glucoside(isoschaftoside) & $\mathrm{C}_{26} \mathrm{H}_{28} \mathrm{O}_{14}$ & 564 & Aerial part & [39] \\
\hline 90 & Viscidulin III-2'-O- $\beta$-D-glucopyranoside & $\mathrm{C}_{23} \mathrm{H}_{22} \mathrm{O}_{14}$ & 522 & Root & [38] \\
\hline 91 & Quercetin 3-glucuronide & $\mathrm{C}_{21} \mathrm{H}_{18} \mathrm{O}_{13}$ & 478 & Whole plant & [39] \\
\hline 92 & Naringenin & $\mathrm{C}_{15} \mathrm{H}_{12} \mathrm{O}_{5}$ & 272 & Whole plant & [39] \\
\hline 93 & Pinocembrin & $\mathrm{C}_{15} \mathrm{H}_{12} \mathrm{O}_{4}$ & 256 & Whole plant & [39] \\
\hline 94 & Isocarthamidin ((2S)-5,7,8,4'-Tetrahydroxyflavanone) & $\mathrm{C}_{15} \mathrm{H}_{12} \mathrm{O}_{6}$ & 288 & $\begin{array}{l}\text { Leaf } \\
\text { Root }\end{array}$ & [39] \\
\hline 95 & Carthamidin (2S)-5,6,7,4'-Tetrahydroxyflavanone) & $\mathrm{C}_{15} \mathrm{H}_{12} \mathrm{O}_{6}$ & 288 & $\begin{array}{l}\text { Leaf } \\
\text { Root }\end{array}$ & [39] \\
\hline 96 & (2S)-5,7,4'-Trihydroxy-6-methoxyflavanone & $\mathrm{C}_{16} \mathrm{H}_{14} \mathrm{O}_{6}$ & 302 & Root & [38] \\
\hline 97 & (+)-Eriodictyol ((2S)-5,7,3',4'-Tetrahydroxyflavanone) & $\mathrm{C}_{15} \mathrm{H}_{12} \mathrm{O}_{6}$ & 288 & Root & [38] \\
\hline 98 & (2S)-5,4'-Dihydroxy-7-methoxyflavanone & $\mathrm{C}_{16} \mathrm{H}_{14} \mathrm{O}_{5}$ & 286 & Aerial part & [38] \\
\hline 99 & Dihydrooroxylin A ((25)-5,7-Dihydroxy-6-methoxyflavanone) & $\mathrm{C}_{16} \mathrm{H}_{14} \mathrm{O}_{5}$ & 286 & Root & [38] \\
\hline 100 & (2S)-7-Hydroxy-5-methoxyflavanone & $\mathrm{C}_{16} \mathrm{H}_{14} \mathrm{O}_{4}$ & 270 & Root & [38] \\
\hline 101 & (2S)-5,7,2',5'-Tetrahydroxyflavanone & $\mathrm{C}_{15} \mathrm{H}_{12} \mathrm{O}_{6}$ & 288 & Root & [38] \\
\hline 102 & (25)-5,7,2',6'-Tetrahydroxyflavanone & $\mathrm{C}_{15} \mathrm{H}_{12} \mathrm{O}_{6}$ & 288 & Root & [37] \\
\hline 103 & (2S)-7,2',6'-Trihydroxy-5-methoxyflavanone & $\mathrm{C}_{16} \mathrm{H}_{14} \mathrm{O}_{6}$ & 302 & Root & [38] \\
\hline 104 & (2R,3R)-3,5,7,2',6'-Pentahydroxyflavanone & $\mathrm{C}_{15} \mathrm{H}_{12} \mathrm{O}_{7}$ & 304 & Root & [37] \\
\hline 106 & Naringenin 7-O-glucoronide & $\mathrm{C}_{21} \mathrm{H}_{20} \mathrm{O}_{11}$ & 448 & Whole plant & [39] \\
\hline 107 & Pinocembrin 7-O-glucoronide & $\mathrm{C}_{21} \mathrm{H}_{20} \mathrm{O}_{10}$ & 432 & Whole plant & [39] \\
\hline 108 & (2S) -5,7,2',5'-Tetrahydroxyflavanone-7-O- $\beta$-D-glucopyranoside & $\mathrm{C}_{21} \mathrm{H}_{22} \mathrm{O}_{10}$ & 434 & Root & [38] \\
\hline 109 & (2S)-5,7-Dihydroxy-6-methoxyflavanone-7-O- $\beta$-D-glucopyranoside & $\mathrm{C}_{22} \mathrm{H}_{22} \mathrm{O}_{10}$ & 446 & Root & [38] \\
\hline 110 & (2S)-5-Hydroxy-6-methoxyflavanone 7-O- $\beta$-D-glucoside & $\mathrm{C}_{22} \mathrm{H}_{24} \mathrm{O}_{10}$ & 448 & Root & [38] \\
\hline 111 & (2S)-5,7,6'-Trihydroxyflavanone 2'-O- $\beta$-D-glucoside & $\mathrm{C}_{21} \mathrm{H}_{22} \mathrm{O}_{11}$ & 450 & Root & [37] \\
\hline 112 & Dihydrobaicalin ((2S)-5,6-Dihydroxyflavanone 7-O- $\beta$-D-glucuronide) & $\mathrm{C}_{21} \mathrm{H}_{20} \mathrm{O}_{11}$ & 448 & Root & [38] \\
\hline 113 & (2S)-5-Hydroxy-6-methoxyflavanone 7-O- $\beta$-D-glucuronide & $\mathrm{C}_{22} \mathrm{H}_{22} \mathrm{O}_{11}$ & 462 & Root & [37] \\
\hline 114 & (2S)-5,6,3',4'-Tetrahydroxyflavanone 7-O- $\beta$-D-glucuronide & $\mathrm{C}_{21} \mathrm{H}_{20} \mathrm{O}_{13}$ & 480 & Aerial part & [38] \\
\hline 115 & Isocarthamidin-7-O- $\beta$-D-glucuronide ((2S)-5,8,4'-Trihydroxyflavanone 7-O- $\beta$-D-glucuronide) & $\mathrm{C}_{21} \mathrm{H}_{20} \mathrm{O}_{12}$ & 464 & Aerial part & [39] \\
\hline 116 & Carthamidin 7-O- $\beta$-D-glucuronide (Dihydroscutellarein 7-O- $\beta$-D-glucuronide, Scutellarin B) & $\mathrm{C}_{21} \mathrm{H}_{20} \mathrm{O}_{12}$ & 464 & Aerial part & [39] \\
\hline 117 & (2S)-5,8,3',4'-Tetrahydroxyflavanone 7-O- $\beta$-D-glucuronide & $\mathrm{C}_{21} \mathrm{H}_{20} \mathrm{O}_{13}$ & 480 & Aerial part & [38] \\
\hline 118 & 4',5,7-Trihydroxy-6-methoxyflavanone & $\mathrm{C}_{16} \mathrm{H}_{15} \mathrm{O}_{5}$ & 287 & Root & [38] \\
\hline 119 & $2^{\prime}, 6^{\prime}, 5,7-T e t r a h y d r o x y f l a v a n o n e$ & $\mathrm{C}_{15} \mathrm{H}_{13} \mathrm{O}_{5}$ & 273 & Root & [38] \\
\hline 120 & Sinapoyl hexoside & $\mathrm{C}_{27} \mathrm{H}_{22} \mathrm{O}_{10}$ & 386 & Whole plan & [39] \\
\hline 121 & Verbascoside & $\mathrm{C}_{29} \mathrm{H}_{36} \mathrm{O}_{15}$ & 624 & Whole plant & [39] \\
\hline 122 & 7-O-Acetylloganic acid & $\mathrm{C}_{28} \mathrm{H}_{26} \mathrm{O}_{10}$ & 402 & Whole plant & [39] \\
\hline 123 & $N^{1}, N^{5}, N^{10}$-Tri-p-(E,E,E)-coumaroylspermidine & $\mathrm{C}_{33} \mathrm{H}_{33} \mathrm{O}_{6} \mathrm{~N}_{3}$ & 567 & Whole plant & [39] \\
\hline 124 & Benzyl alcohol & $\mathrm{C}_{7} \mathrm{H}_{8} \mathrm{O}$ & 108 & Root & [36] \\
\hline 125 & 4'-(ß-D-glucopyranosyloxy)-3,3',5,5'-tetramethoxy-9,9'-epoxylignane-4, 7'-diol & $\mathrm{C}_{30} \mathrm{H}_{45} \mathrm{O}_{13}$ & 613 & Root & [36] \\
\hline 126 & 4'-(ß-D-glucopyranosyloxy)-3,3',5'-trimethoxy-9,9'-epoxylignane-4, 7'-diol & $\mathrm{C}_{29} \mathrm{H}_{43} \mathrm{O}_{12}$ & 583 & Root & [36] \\
\hline 127 & 4'-(ß-D-glucopyranosyloxy)-3,3-dimethoxy-9,9'-epoxylignane-4,7'-diol & $\mathrm{C}_{28} \mathrm{H}_{41} \mathrm{O}_{11}$ & 553 & Root & [36] \\
\hline 128 & Lutein & $\mathrm{C}_{40} \mathrm{H}_{55} \mathrm{O}_{2}$ & 567 & Root & [36] \\
\hline 129 & $\beta$-Carotene & $\mathrm{C}_{40} \mathrm{H}_{56}$ & 536 & Root & [36] \\
\hline 130 & 2,6,2',4'-Tetrahydroxy-6'-methoxychalcone & $\mathrm{C}_{16} \mathrm{H}_{14} \mathrm{O}_{6}$ & 302 & Root & [38] \\
\hline
\end{tabular}


Table 1 (continued)

\begin{tabular}{|c|c|c|c|c|c|}
\hline No. & Name & Molecular formula & Weight & Plant part & Ref. \\
\hline 131 & $8,8^{\prime \prime}$-Bibaicalein & $\mathrm{C}_{30} \mathrm{H}_{18} \mathrm{O}_{10}$ & 538 & Root & [38] \\
\hline 132 & 5,6,8-Trimethoxy-3',4'-methylenedioxyflavone 7-O- $\beta$-D-glucoside & $\mathrm{C}_{26} \mathrm{H}_{28} \mathrm{O}_{12}$ & 532 & Root & [38] \\
\hline 133 & 3,5,8-Trimethoxy-3',4'-methylenedioxyflavone 7-O- $\beta$-D-glucoside & $\mathrm{C}_{26} \mathrm{H}_{28} \mathrm{O}_{12}$ & 532 & Root & [38] \\
\hline 134 & Delphinidin 3-O-(6-O-malonyl)- $\beta$-D-glucoside-5-O- $\beta$-D-glucoside & $\mathrm{C}_{30} \mathrm{H}_{33} \mathrm{O}_{20}$ & 713 & Flower & [38] \\
\hline 135 & Salidroside (4-Hydroxy- $\beta$-phenylethyl- $\beta$-D-glucoside) & $\mathrm{C}_{14} \mathrm{H}_{20} \mathrm{O}_{7}$ & 300 & Hairy Root & [38] \\
\hline 136 & Darendoside B & $\mathrm{C}_{21} \mathrm{H}_{32} \mathrm{O}_{12}$ & 476 & Root & [38] \\
\hline 137 & $\begin{array}{l}\text { Martynoside (2-(3-Hydroxy-4-methoxyphenyl) ethyl-1-O-a-L-rhamnosyl }(1 \rightarrow 3)-\beta-D-(4- \\
\text { feruloyl)-glucoside) }\end{array}$ & $\mathrm{C}_{31} \mathrm{H}_{40} \mathrm{O}_{15}$ & 652 & $\begin{array}{l}\text { Hairy Root } \\
\text { Root }\end{array}$ & [38] \\
\hline 138 & Acteoside & $\mathrm{C}_{29} \mathrm{H}_{36} \mathrm{O}_{15}$ & 624 & $\begin{array}{l}\text { Hairy Root } \\
\text { Root }\end{array}$ & [37] \\
\hline 139 & Isomartynoside & $\mathrm{C}_{31} \mathrm{H}_{40} \mathrm{O}_{15}$ & 652 & Root & [38] \\
\hline 140 & Leucosceptoside A & $\mathrm{C}_{30} \mathrm{H}_{38} \mathrm{O}_{15}$ & 638 & $\begin{array}{l}\text { Hairy Root } \\
\text { Root }\end{array}$ & [38] \\
\hline 141 & Cistanoside D & $\mathrm{C}_{31} \mathrm{H}_{40} \mathrm{O}_{15}$ & 652 & Root & [37] \\
\hline 142 & Darendoside A & $\mathrm{C}_{19} \mathrm{H}_{28} \mathrm{O}_{11}$ & 432 & Root & [38] \\
\hline 143 & Stigmasterol & $\mathrm{C}_{29} \mathrm{H}_{48} \mathrm{O}$ & 412 & Root & [38] \\
\hline 144 & $\beta$-Sitosterol & $\mathrm{C}_{29} \mathrm{H}_{50} \mathrm{O}$ & 414 & Root & [36] \\
\hline 145 & Daucosterin & $\mathrm{C}_{35} \mathrm{H}_{60} \mathrm{O}_{6}$ & 576 & Root & [38] \\
\hline 146 & 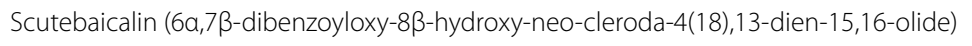 & $\mathrm{C}_{34} \mathrm{H}_{38} \mathrm{O}_{7}$ & 558 & Aerial part & [38] \\
\hline 147 & Pellitorine & $\mathrm{C}_{14} \mathrm{H}_{25} \mathrm{NO}$ & 223 & Root & [38] \\
\hline 148 & (E)-4-[(2-methylpropyl) amino]-4-oxo-2-butenoic acid & $\mathrm{C}_{8} \mathrm{H}_{13} \mathrm{NO}_{3}$ & 171 & Root & [38] \\
\hline 149 & Dihydropiperlonguminine & $\mathrm{C}_{16} \mathrm{H}_{21} \mathrm{NO}_{3}$ & 275 & Root & [38] \\
\hline 150 & Futoamide & $\mathrm{C}_{18} \mathrm{H}_{23} \mathrm{NO}_{3}$ & 301 & Root & [38] \\
\hline 151 & Piperlonguminine & $\mathrm{C}_{16} \mathrm{H}_{19} \mathrm{NO}_{3}$ & 273 & Root & [38] \\
\hline 152 & Benzoic acid & $\mathrm{C}_{7} \mathrm{H}_{6} \mathrm{O}_{2}$ & 122 & Root & [38] \\
\hline 153 & Phenyl acetic acid & $\mathrm{C}_{8} \mathrm{H}_{8} \mathrm{O}_{2}$ & 136 & Root & [38] \\
\hline 154 & Syringaldehyde & $\mathrm{C}_{9} \mathrm{H}_{10} \mathrm{O}_{4}$ & 182 & Root & [38] \\
\hline 155 & 4-O- $\beta$-D-glucosyl-trans-p-coumaric acid & $\mathrm{C}_{15} \mathrm{H}_{18} \mathrm{O}_{8}$ & 326 & Root & [38] \\
\hline 156 & Ferulic acid methyl ester & $\mathrm{C}_{11} \mathrm{H}_{12} \mathrm{O}_{4}$ & 208 & Root & [38] \\
\hline 157 & 4-O- $\beta$-D-glucosyl-cis-p-coumaric acid & $\mathrm{C}_{15} \mathrm{H}_{18} \mathrm{O}_{8}$ & 326 & Root & [38] \\
\hline 158 & Vanillin & $\mathrm{C}_{8} \mathrm{H}_{8} \mathrm{O}_{3}$ & 152 & Root & [38] \\
\hline 159 & (+)-Crotepoxide & $\mathrm{C}_{18} \mathrm{H}_{18} \mathrm{O}_{8}$ & 362 & Root & [38] \\
\hline 160 & (+)-Syringaresinol-O- $\beta$-D-glucoside & $\mathrm{C}_{28} \mathrm{H}_{36} \mathrm{O}_{13}$ & 580 & Root & [38] \\
\hline
\end{tabular}

coccus-shaped, non-spore-forming actinobacterium. Whether the existence and growth of the bacteria will affect the synthesis of active ingredients, it maybe as a new research point of SB.

\section{Pharmacological effects (Shown in Table 2) Anti-inflammatory}

According to clinical applications, SB is widely used in anti-inflammatory effect. Many diseases can lead to inflammation. In the process of inflammation, all kinds of inflammatory mediators will be produced, such as iNOS, COX-2, IL-6, TNF- $\alpha$, etc. These mediators can aggravate the inflammatory and then further expand into a vicious cycle [58]. It has been confirmed that the flavonoids of SB can inhibit the release of many inflammatory factors [59]. A new polysaccharide was extracted from $\mathrm{SB}$, and it also could significantly inhibit the level of pro-inflammatory cytokines in serum, including TNF- $\alpha$, IL-1 $\beta$, IL-18, etc. [51]. For inflammatory cells, baicalein can induce their apoptosis, thus reducing the release of inflammatory factors and the invasion of normal cells under the infiltration of inflammatory cells [60]. Inflammation is associated with the expression of many proteins, such as COX2 , vimentin, annexin A1, annexin A2, etc. And flavonoids in SB can inhibit the expression of these proteins to achieve anti-inflammatory effect [61]. The signaling pathways of PPAR $\gamma$ and NF-kB were also associated with the occurrence of inflammatory. SBE could inhibit LPS induced AET II cell inflammatory response 


\begin{tabular}{|c|c|c|c|c|c|c|c|c|c|c|}
\hline 1 & $\mathrm{R}_{1}=\mathrm{H}$ & $\mathrm{R}_{2}=\mathrm{OH}$ & $\mathrm{R}_{3}=\mathrm{OH}$ & $\mathrm{R}_{4}=\mathrm{OH}$ & $\mathrm{R}_{5}=\mathrm{H}$ & $\mathrm{R}_{6}=\mathrm{H}$ & $\mathrm{R}_{7}=\mathrm{H}$ & $\mathrm{R}_{8}=\mathrm{H}$ & $\mathrm{R}_{9}=\mathrm{H}$ & $\mathrm{R}_{10}=\mathrm{H}$ \\
\hline 2 & $\mathrm{R}_{1}=\mathrm{H}$ & $\mathrm{R}_{2}=\mathrm{OCH}_{3}$ & $\mathrm{R}_{3}=\mathrm{OH}$ & $\mathrm{R}_{4}=\mathrm{OH}$ & $\mathrm{R}_{5}=\mathrm{H}$ & $\mathrm{R}_{6}=\mathrm{H}$ & $\mathrm{R}_{7}=\mathrm{H}$ & $\mathrm{R}_{8}=\mathrm{H}$ & $\mathrm{R}_{9}=\mathrm{H}$ & $\mathrm{R}_{10}=\mathrm{H}$ \\
\hline 3 & $\mathrm{R}_{1}=\mathrm{H}$ & $\mathrm{R}_{2}=\mathrm{OH}$ & $\mathrm{R}_{3}=\mathrm{OH}$ & $\mathrm{R}_{4}=\mathrm{OH}$ & $\mathrm{R}_{5}=\mathrm{OH}$ & $\mathrm{R}_{6}=\mathrm{H}$ & $\mathrm{R}_{7}=\mathrm{H}$ & $\mathrm{R}_{8}=\mathrm{H}$ & $\mathrm{R}_{9}=\mathrm{OH}$ & $\mathrm{R}_{10}=\mathrm{H}$ \\
\hline 4 & $\mathrm{R}_{1}=\mathrm{H}$ & $\mathrm{R}_{2}=\mathrm{OH}$ & $\mathrm{R}_{3}=\mathrm{OH}$ & $\mathrm{R}_{4}=\mathrm{OH}$ & $\mathrm{R}_{5}=\mathrm{H}$ & $\mathrm{R}_{6}=\mathrm{H}$ & $\mathrm{R}_{7}=\mathrm{H}$ & $\mathrm{R}_{8}=\mathrm{OCH}_{3}$ & $\mathrm{R}_{9}=\mathrm{H}$ & $\mathrm{R}_{10}=\mathrm{H}$ \\
\hline 5 & $\mathrm{R}_{1}=\mathrm{H}$ & $\mathrm{R}_{2}=\mathrm{OH}$ & $\mathrm{R}_{3}=\mathrm{OCH}_{3}$ & $\mathrm{R}_{4}=\mathrm{OH}$ & $\mathrm{R}_{5}=\mathrm{H}$ & $\mathrm{R}_{6}=\mathrm{H}$ & $\mathrm{R}_{7}=\mathrm{H}$ & $\mathrm{R}_{8}=\mathrm{H}$ & $\mathrm{R}_{9}=\mathrm{H}$ & $\mathrm{R}_{10}=\mathrm{H}$ \\
\hline 6 & $\mathrm{R}_{1}=\mathrm{H}$ & $\mathrm{R}_{2}=\mathrm{OH}$ & $\mathrm{R}_{3}=\mathrm{OCH}_{3}$ & $\mathrm{R}_{4}=\mathrm{OH}$ & $\mathrm{R}_{5}=\mathrm{H}$ & $\mathrm{R}_{6}=\mathrm{H}$ & $\mathrm{R}_{7}=\mathrm{H}$ & $\mathrm{R}_{8}=\mathrm{H}$ & $\mathrm{R}_{9}=\mathrm{H}$ & $\mathrm{R}_{10}=\mathrm{OH}$ \\
\hline 7 & $\mathrm{R}_{1}=\mathrm{H}$ & $\mathrm{R}_{2}=\mathrm{OH}$ & $\mathrm{R}_{3}=\mathrm{OCH}_{3}$ & $\mathrm{R}_{4}=\mathrm{OH}$ & $\mathrm{R}_{5}=\mathrm{H}$ & $\mathrm{R}_{6}=\mathrm{H}$ & $\mathrm{R}_{7}=\mathrm{H}$ & $\mathrm{R}_{8}=\mathrm{OH}$ & $\mathrm{R}_{9}=\mathrm{H}$ & $\mathrm{R}_{10}=\mathrm{H}$ \\
\hline 8 & $\mathrm{R}_{1}=\mathrm{OCH}_{3}$ & $\mathrm{R}_{2}=\mathrm{OH}$ & $\mathrm{R}_{3}=\mathrm{OCH}_{3}$ & $\mathrm{R}_{4}=\mathrm{OH}$ & $\mathrm{R}_{5}=\mathrm{H}$ & $\mathrm{R}_{6}=\mathrm{H}$ & $\mathrm{R}_{7}=\mathrm{H}$ & $\mathrm{R}_{8}=\mathrm{H}$ & $\mathrm{R}_{9}=\mathrm{H}$ & $\mathrm{R}_{10}=\mathrm{H}$ \\
\hline 9 & $\mathrm{R}_{1}=\mathrm{OCH}_{3}$ & $\mathrm{R}_{2}=\mathrm{OH}$ & $\mathrm{R}_{3}=\mathrm{OCH}_{3}$ & $\mathrm{R}_{4}=\mathrm{OH}$ & $\mathrm{R}_{5}=\mathrm{H}$ & $\mathrm{R}_{6}=\mathrm{H}$ & $\mathrm{R}_{7}=\mathrm{H}$ & $\mathrm{R}_{8}=\mathrm{H}$ & $\mathrm{R}_{9}=\mathrm{H}$ & $\mathrm{R}_{10}=\mathrm{OH}$ \\
\hline 10 & $\mathrm{R}_{1}=\mathrm{OH}$ & $\mathrm{R}_{2}=\mathrm{OCH}_{3}$ & $\mathrm{R}_{3}=\mathrm{OCH}_{3}$ & $\mathrm{R}_{4}=\mathrm{OH}$ & $\mathrm{R}_{5}=\mathrm{OH}$ & $\mathrm{R}_{6}=\mathrm{H}$ & $\mathrm{R}_{7}=\mathrm{H}$ & $\mathrm{R}_{8}=\mathrm{H}$ & $\mathrm{R}_{9}=\mathrm{H}$ & $\mathrm{R}_{10}=\mathrm{H}$ \\
\hline 11 & $\mathrm{R}_{1}=\mathrm{OH}$ & $\mathrm{R}_{2}=\mathrm{OCH}_{3}$ & $\mathrm{R}_{3}=\mathrm{OCH}_{3}$ & $\mathrm{R}_{4}=\mathrm{OH}$ & $\mathrm{R}_{5}=\mathrm{H}$ & $\mathrm{R}_{6}=\mathrm{H}$ & $\mathrm{R}_{7}=\mathrm{H}$ & $\mathrm{R}_{8}=\mathrm{H}$ & $\mathrm{R}_{9}=\mathrm{H}$ & $\mathrm{R}_{10}=\mathrm{OH}$ \\
\hline 12 & $\mathrm{R}_{1}=\mathrm{OCH}_{3}$ & $\mathrm{R}_{2}=\mathrm{OCH}_{3}$ & $\mathrm{R}_{3}=\mathrm{OCH}_{3}$ & $\mathrm{R}_{4}=\mathrm{OH}$ & $\mathrm{R}_{5}=\mathrm{H}$ & $\mathrm{R}_{6}=\mathrm{H}$ & $\mathrm{R}_{7}=\mathrm{H}$ & $\mathrm{R}_{8}=\mathrm{H}$ & $\mathrm{R}_{9}=\mathrm{H}$ & $\mathrm{R}_{10}=\mathrm{OH}$ \\
\hline 13 & $\mathrm{R}_{1}=\mathrm{OCH}_{3}$ & $\mathrm{R}_{2}=\mathrm{OCH}_{3}$ & $\mathrm{R}_{3}=\mathrm{OCH}_{3}$ & $\mathrm{R}_{4}=\mathrm{OH}$ & $\mathrm{R}_{5}=\mathrm{H}$ & $\mathrm{R}_{6}=\mathrm{H}$ & $\mathrm{R}_{7}=\mathrm{OH}$ & $\mathrm{R}_{8}=\mathrm{H}$ & $\mathrm{R}_{9}=\mathrm{H}$ & $\mathrm{R}_{10}=\mathrm{OH}$ \\
\hline 14 & $\mathrm{R}_{1}=\mathrm{OCH}_{3}$ & $\mathrm{R}_{2}=\mathrm{OCH}_{3}$ & $\mathrm{R}_{3}=\mathrm{OCH}_{3}$ & $\mathrm{R}_{4}=\mathrm{OH}$ & $\mathrm{R}_{5}=\mathrm{H}$ & $\mathrm{R}_{6}=\mathrm{OCH}_{3}$ & $\mathrm{R}_{7}=\mathrm{H}$ & $\mathrm{R}_{8}=\mathrm{H}$ & $\mathrm{R}_{9}=\mathrm{H}$ & $\mathrm{R}_{10}=\mathrm{OH}$ \\
\hline 15 & $\mathrm{R}_{1}=\mathrm{H}$ & $\mathrm{R}_{2}=\mathrm{OCH}_{3}$ & $\mathrm{R}_{3}=\mathrm{OCH}_{3}$ & $\mathrm{R}_{4}=\mathrm{OH}$ & $\mathrm{R}_{5}=\mathrm{H}$ & $\mathrm{R}_{6}=\mathrm{H}$ & $\mathrm{R}_{7}=\mathrm{OCH}_{3}$ & $\mathrm{R}_{8}=\mathrm{OH}$ & $\mathrm{R}_{9}=\mathrm{OCH}_{3}$ & $\mathrm{R}_{10}=\mathrm{H}$ \\
\hline 16 & $\mathrm{R}_{1}=\mathrm{OCH}_{3}$ & $\mathrm{R}_{2}=\mathrm{OCH}_{3}$ & $\mathrm{R}_{3}=\mathrm{OCH}_{3}$ & $\mathrm{R}_{4}=\mathrm{OH}$ & $\mathrm{R}_{5}=\mathrm{H}$ & $\mathrm{R}_{6}=\mathrm{OH}$ & $\mathrm{R}_{7}=\mathrm{OCH}_{3}$ & $\mathrm{R}_{8}=\mathrm{H}$ & $\mathrm{R}_{9}=\mathrm{H}$ & $\mathrm{R}_{10}=\mathrm{H}$ \\
\hline 17 & $\mathrm{R}_{1}=\mathrm{H}$ & $\mathrm{R}_{2}=\mathrm{OH}$ & $\mathrm{R}_{3}=\mathrm{H}$ & $\mathrm{R}_{4}=\mathrm{OH}$ & $\mathrm{R}_{5}=\mathrm{H}$ & $\mathrm{R}_{6}=\mathrm{H}$ & $\mathrm{R}_{7}=\mathrm{H}$ & $\mathrm{R}_{8}=\mathrm{H}$ & $\mathrm{R}_{9}=\mathrm{H}$ & $\mathrm{R}_{10}=\mathrm{H}$ \\
\hline 18 & $\mathrm{R}_{1}=\mathrm{OH}$ & $\mathrm{R}_{2}=\mathrm{OH}$ & $\mathrm{R}_{3}=\mathrm{H}$ & $\mathrm{R}_{4}=\mathrm{OH}$ & $\mathrm{R}_{5}=\mathrm{H}$ & $\mathrm{R}_{6}=\mathrm{H}$ & $\mathrm{R}_{7}=\mathrm{H}$ & $\mathrm{R}_{8}=\mathrm{H}$ & $\mathrm{R}_{9}=\mathrm{H}$ & $\mathrm{R}_{10}=\mathrm{H}$ \\
\hline 19 & $\mathrm{R}_{1}=\mathrm{OH}$ & $\mathrm{R}_{2}=\mathrm{OH}$ & $\mathrm{R}_{3}=\mathrm{H}$ & $\mathrm{R}_{4}=\mathrm{OH}$ & $\mathrm{R}_{5}=\mathrm{H}$ & $\mathrm{R}_{6}=\mathrm{H}$ & $\mathrm{R}_{7}=\mathrm{H}$ & $\mathrm{R}_{8}=\mathrm{OH}$ & $\mathrm{R}_{9}=\mathrm{H}$ & $\mathrm{R}_{10}=\mathrm{H}$ \\
\hline 20 & $\mathrm{R}_{1}=\mathrm{H}$ & $\mathrm{R}_{2}=\mathrm{OH}$ & $\mathrm{R}_{3}=\mathrm{H}$ & $\mathrm{R}_{4}=\mathrm{OH}$ & $\mathrm{R}_{5}=\mathrm{H}$ & $\mathrm{R}_{6}=\mathrm{OCH}_{3}$ & $\mathrm{R}_{7}=\mathrm{H}$ & $\mathrm{R}_{8}=\mathrm{H}$ & $\mathrm{R}_{9}=\mathrm{H}$ & $\mathrm{R}_{10}=\mathrm{OH}$ \\
\hline 21 & $\mathrm{R}_{1}=\mathrm{OCH}_{3}$ & $\mathrm{R}_{2}=\mathrm{OH}$ & $\mathrm{R}_{3}=\mathrm{H}$ & $\mathrm{R}_{4}=\mathrm{OH}$ & $\mathrm{R}_{5}=\mathrm{H}$ & $\mathrm{R}_{6}=\mathrm{H}$ & $\mathrm{R}_{7}=\mathrm{H}$ & $\mathrm{R}_{8}=\mathrm{OH}$ & $\mathrm{R}_{9}=\mathrm{H}$ & $\mathrm{R}_{10}=\mathrm{H}$ \\
\hline 22 & $\mathrm{R}_{1}=\mathrm{H}$ & $\mathrm{R}_{2}=\mathrm{OH}$ & $\mathrm{R}_{3}=\mathrm{H}$ & $\mathrm{R}_{4}=\mathrm{OH}$ & $\mathrm{R}_{5}=\mathrm{H}$ & $\mathrm{R}_{6}=\mathrm{H}$ & $\mathrm{R}_{7}=\mathrm{H}$ & $\mathrm{R}_{8}=\mathrm{H}$ & $\mathrm{R}_{9}=\mathrm{H}$ & $\mathrm{R}_{10}=\mathrm{OH}$ \\
\hline 23 & $\mathrm{R}_{1}=\mathrm{H}$ & $\mathrm{R}_{2}=\mathrm{OH}$ & $\mathrm{R}_{3}=\mathrm{H}$ & $\mathrm{R}_{4}=\mathrm{OH}$ & $\mathrm{R}_{5}=\mathrm{H}$ & $\mathrm{R}_{6}=\mathrm{H}$ & $\mathrm{R}_{7}=\mathrm{H}$ & $\mathrm{R}_{8}=\mathrm{H}$ & $\mathrm{R}_{9}=\mathrm{OH}$ & $\mathrm{R}_{10}=\mathrm{OH}$ \\
\hline 24 & $\mathrm{R}_{1}=\mathrm{H}$ & $\mathrm{R}_{2}=\mathrm{OH}$ & $\mathrm{R}_{3}=\mathrm{OH}$ & $\mathrm{R}_{4}=\mathrm{OH}$ & $\mathrm{R}_{5}=\mathrm{H}$ & $\mathrm{R}_{6}=\mathrm{H}$ & $\mathrm{R}_{7}=\mathrm{H}$ & $\mathrm{R}_{8}=\mathrm{OH}$ & $\mathrm{R}_{9}=\mathrm{OH}$ & $\mathrm{R}_{10}=\mathrm{H}$ \\
\hline 25 & $\mathrm{R}_{\mathrm{l}}=\mathrm{H}$ & $\mathrm{R}_{2}=\mathrm{OCH}_{3}$ & $\mathrm{R}_{3}=\mathrm{OCH}_{3}$ & $\mathrm{R}_{4}=\mathrm{OH}$ & $\mathrm{R}_{5}=\mathrm{H}$ & $\mathrm{R}_{6}=\mathrm{H}$ & $\mathrm{R}_{7}=\mathrm{OCH}_{3}$ & $\mathrm{R}_{8}=\mathrm{H}$ & $\mathrm{R}_{9}=\mathrm{H}$ & $\mathrm{R}_{10}=\mathrm{H}$ \\
\hline 26 & $\mathrm{R}_{1}=\mathrm{H}$ & $\mathrm{R}_{2}=\mathrm{OH}$ & $\mathrm{R}_{3}=\mathrm{H}$ & $\mathrm{R}_{4}=\mathrm{OH}$ & $\mathrm{R}_{5}=\mathrm{H}$ & $\mathrm{R}_{6}=\mathrm{H}$ & $\mathrm{R}_{7}=\mathrm{OH}$ & $\mathrm{R}_{8}=\mathrm{H}$ & $\mathrm{R}_{9}=\mathrm{H}$ & $\mathrm{R}_{10}=\mathrm{OH}$ \\
\hline 27 & $\mathrm{R}_{1}=\mathrm{H}$ & $\mathrm{R}_{2}=\mathrm{OH}$ & $\mathrm{R}_{3}=\mathrm{H}$ & $\mathrm{R}_{4}=\mathrm{OH}$ & $\mathrm{R}_{5}=\mathrm{H}$ & $\mathrm{R}_{6}=\mathrm{H}$ & $\mathrm{R}_{7}=\mathrm{H}$ & $\mathrm{R}_{8}=\mathrm{OH}$ & $\mathrm{R}_{9}=\mathrm{OH}$ & $\mathrm{R}_{10}=\mathrm{H}$ \\
\hline 28 & $\mathrm{R}_{1}=\mathrm{H}$ & $\mathrm{R}_{2}=\mathrm{OH}$ & $\mathrm{R}_{3}=\mathrm{H}$ & $\mathrm{R}_{4}=\mathrm{OH}$ & $\mathrm{R}_{5}=\mathrm{H}$ & $\mathrm{R}_{6}=\mathrm{OH}$ & $\mathrm{R}_{7}=\mathrm{H}$ & $\mathrm{R}_{8}=\mathrm{H}$ & $\mathrm{R}_{9}=\mathrm{H}$ & $\mathrm{R}_{10}=\mathrm{OH}$ \\
\hline 29 & $\mathrm{R}_{1}=\mathrm{H}$ & $\mathrm{R}_{2}=\mathrm{OH}$ & $\mathrm{R}_{3}=\mathrm{H}$ & $\mathrm{R}_{4}=\mathrm{OH}$ & $\mathrm{R}_{5}=\mathrm{H}$ & $\mathrm{R}_{6}=\mathrm{OCH}_{3}$ & $\mathrm{R}_{7}=\mathrm{H}$ & $\mathrm{R}_{8}=\mathrm{H}$ & $\mathrm{R}_{9}=\mathrm{H}$ & $\mathrm{R}_{10}=\mathrm{OH}$ \\
\hline 30 & $\mathrm{R}_{1}=\mathrm{OCH}_{3}$ & $\mathrm{R}_{2}=\mathrm{OH}$ & $\mathrm{R}_{3}=\mathrm{OCH}_{3}$ & $\mathrm{R}_{4}=\mathrm{OH}$ & $\mathrm{R}_{5}=\mathrm{H}$ & $\mathrm{R}_{6}=\mathrm{OCH}_{3}$ & $\mathrm{R}_{7}=\mathrm{OCH}_{3}$ & $\mathrm{R}_{8}=\mathrm{H}$ & $\mathrm{R}_{9}=\mathrm{H}$ & $\mathrm{R}_{10}=\mathrm{H}$ \\
\hline 31 & $\mathrm{R}_{1}=\mathrm{OCH}_{3}$ & $\mathrm{R}_{2}=\mathrm{OH}$ & $\mathrm{R}_{3}=\mathrm{H}$ & $\mathrm{R}_{4}=\mathrm{OH}$ & $\mathrm{R}_{5}=\mathrm{H}$ & $\mathrm{R}_{6}=\mathrm{H}$ & $\mathrm{R}_{7}=\mathrm{H}$ & $\mathrm{R}_{8}=\mathrm{H}$ & $\mathrm{R}_{9}=\mathrm{H}$ & $\mathrm{R}_{10}=\mathrm{H}$ \\
\hline 32 & $\mathrm{R}_{1}=\mathrm{OCH}_{3}$ & $\mathrm{R}_{2}=\mathrm{OCH}_{3}$ & $\mathrm{R}_{3}=\mathrm{OCH}_{3}$ & $\mathrm{R}_{4}=\mathrm{OH}$ & $\mathrm{R}_{5}=\mathrm{OH}$ & $\mathrm{R}_{6}=\mathrm{H}$ & $\mathrm{R}_{7}=\mathrm{H}$ & $\mathrm{R}_{8}=\mathrm{OH}$ & $\mathrm{R}_{9}=\mathrm{H}$ & $\mathrm{R}_{10}=\mathrm{H}$ \\
\hline 33 & $\mathrm{R}_{1}=\mathrm{OCH}_{3}$ & $\mathrm{R}_{2}=\mathrm{OH}$ & $\mathrm{R}_{3}=\mathrm{H}$ & $\mathrm{R}_{4}=\mathrm{OH}$ & $\mathrm{R}_{5}=\mathrm{H}$ & $\mathrm{R}_{6}=\mathrm{H}$ & $\mathrm{R}_{7}=\mathrm{H}$ & $\mathrm{R}_{8}=\mathrm{H}$ & $\mathrm{R}_{9}=\mathrm{H}$ & $\mathrm{R}_{10}=\mathrm{OH}$ \\
\hline & $\mathrm{R}_{1}=\mathrm{OCH}_{3}$ & $\mathrm{R}_{2}=\mathrm{OH}$ & $\mathrm{R}_{3}=\mathrm{H}$ & $\mathrm{R}_{4}=\mathrm{OH}$ & $\mathrm{R}_{5}=\mathrm{H}$ & $\mathrm{R}_{6}=\mathrm{OCH}_{3}$ & $\mathrm{R}_{7}=\mathrm{H}$ & $\mathrm{R}_{8}=\mathrm{H}$ & $\mathrm{R}_{9}=\mathrm{H}$ & $\mathrm{R}_{10}=\mathrm{OH}$ \\
\hline
\end{tabular}

by inhibiting NF- $\mathrm{kB}$, MAPK and phosphatidylinositol-3 kinase signaling pathways [62], and increased the cell viability. To sum up, SB play an anti-inflammatory role mainly by inhibiting the release of inflammatory factors and the expression of inflammatory related proteins.

\section{Antibacterial and antimicrobial}

There were studies showed that SB also has antibacterial and anti microbial effects. The SBWE could significantly inhibit the reproduction of Toxoplasma gondii in 24, 48 and $72 \mathrm{~h}$ [63]. Norwogonin has strong inhibitory effect on multidrug resistant Acinetobacter baumannii [64]. SB 


\begin{tabular}{|c|c|c|c|c|c|c|c|c|c|c|}
\hline 35 & $\mathrm{R}_{1}=\mathrm{OCH}_{3}$ & $\mathrm{R}_{2}=\mathrm{OH}$ & $\mathrm{R}_{3}=\mathrm{H}$ & $\mathrm{R}_{4}=\mathrm{OH}$ & $\mathrm{R}_{s}=\mathrm{H}$ & $\mathrm{R}_{6}=\mathrm{OCH}_{3}$ & $\mathrm{R}_{7}=\mathrm{OH}$ & $\mathrm{R}_{8}=\mathrm{H}$ & $\mathrm{R}_{9}=\mathrm{H}$ & $\mathrm{R}_{10}=\mathrm{OH}$ \\
\hline 36 & $\mathrm{R}_{1}=\mathrm{H}$ & $\mathrm{R}_{2}=\mathrm{OH}$ & $\mathrm{R}_{3}=\mathrm{H}$ & $\mathrm{R}_{4}=\mathrm{OH}$ & $\mathrm{R}_{5}=\mathrm{H}$ & $\mathrm{R}_{6}=\mathrm{OCH}_{3}$ & $\mathrm{R}_{7}=\mathrm{H}$ & $\mathrm{R}_{8}=\mathrm{H}$ & $\mathrm{R}_{9}=\mathrm{H}$ & $\mathrm{R}_{10}=\mathrm{OH}$ \\
\hline 37 & $\mathrm{R}_{1}=\mathrm{OCH}_{3}$ & $\mathrm{R}_{2}=\mathrm{OH}$ & $\mathrm{R}_{3}=\mathrm{H}$ & $\mathrm{R}_{4}=\mathrm{OH}$ & $\mathrm{R}_{s}=\mathrm{H}$ & $\mathrm{R}_{6}=\mathrm{OCH}_{3}$ & $\mathrm{R}_{7}=\mathrm{H}$ & $\mathrm{R}_{8}=\mathrm{H}$ & $\mathrm{R}_{9}=\mathrm{OCH}_{3}$ & $\mathrm{R}_{10}=\mathrm{OCH}_{3}$ \\
\hline 38 & $\mathrm{R}_{1}=\mathrm{H}$ & $\mathrm{R}_{2}=\mathrm{OCH}_{3}$ & $\mathrm{R}_{3}=\mathrm{H}$ & $\mathrm{R}_{4}=\mathrm{OH}$ & $\mathrm{R}_{s}=\mathrm{H}$ & $\mathrm{R}_{6}=\mathrm{H}$ & $\mathrm{R}_{7}=\mathrm{H}$ & $\mathrm{R}_{8}=\mathrm{H}$ & $\mathrm{R}_{9}=\mathrm{H}$ & $\mathrm{R}_{10}=\mathrm{H}$ \\
\hline 39 & $\mathrm{R}_{1}=\mathrm{OH}$ & $\mathrm{R}_{2}=\mathrm{OCH}_{3}$ & $\mathrm{R}_{3}=\mathrm{H}$ & $\mathrm{R}_{4}=\mathrm{OH}$ & $\mathrm{R}_{s}=\mathrm{H}$ & $\mathrm{R}_{6}=\mathrm{H}$ & $\mathrm{R}_{7}=\mathrm{H}$ & $\mathrm{R}_{8}=\mathrm{H}$ & $\mathrm{R}_{9}=\mathrm{H}$ & $\mathrm{R}_{10}=\mathrm{H}$ \\
\hline 40 & $\mathrm{R}_{1}=\mathrm{H}$ & $\mathrm{R}_{2}=\mathrm{OCH}_{3}$ & $\mathrm{R}_{3}=\mathrm{H}$ & $\mathrm{R}_{4}=\mathrm{OH}$ & $\mathrm{R}_{s}=\mathrm{H}$ & $\mathrm{R}_{6}=\mathrm{H}$ & $\mathrm{R}_{7}=\mathrm{H}$ & $\mathrm{R}_{8}=\mathrm{OH}$ & $\mathrm{R}_{9}=\mathrm{H}$ & $\mathrm{R}_{10}=\mathrm{H}$ \\
\hline 41 & $\mathrm{R}_{1}=\mathrm{OH}$ & $\mathrm{R}_{2}=\mathrm{OCH}_{3}$ & $\mathrm{R}_{3}=\mathrm{H}$ & $\mathrm{R}_{4}=\mathrm{OH}$ & $\mathrm{R}_{5}=\mathrm{H}$ & $\mathrm{R}_{6}=\mathrm{H}$ & $\mathrm{R}_{7}=\mathrm{H}$ & $\mathrm{R}_{8}=\mathrm{H}$ & $\mathrm{R}_{9}=\mathrm{H}$ & $\mathrm{R}_{10}=\mathrm{OH}$ \\
\hline 42 & $\mathrm{R}_{1}=\mathrm{OCH}_{3}$ & $\mathrm{R}_{2}=\mathrm{OCH}_{3}$ & $\mathrm{R}_{3}=\mathrm{H}$ & $\mathrm{R}_{4}=\mathrm{OH}$ & $\mathrm{R}_{s}=\mathrm{H}$ & $\mathrm{R}_{6}=\mathrm{H}$ & $\mathrm{R}_{7}=\mathrm{H}$ & $\mathrm{R}_{8}=\mathrm{H}$ & $\mathrm{R}_{9}=\mathrm{H}$ & $\mathrm{R}_{10}=\mathrm{H}$ \\
\hline 43 & $\mathrm{R}_{1}=\mathrm{H}$ & $\mathrm{R}_{2}=\mathrm{OH}$ & $\mathrm{R}_{3}=\mathrm{H}$ & $\mathrm{R}_{4}=\mathrm{OH}$ & $\mathrm{R}_{s}=\mathrm{H}$ & $\mathrm{R}_{6}=\mathrm{OH}$ & $\mathrm{R}_{7}=\mathrm{H}$ & $\mathrm{R}_{8}=\mathrm{H}$ & $\mathrm{R}_{9}=\mathrm{H}$ & $\mathrm{R}_{10}=\mathrm{OH}$ \\
\hline 44 & $\mathrm{R}_{1}=\mathrm{OCH}_{3}$ & $\mathrm{R}_{2}=\mathrm{OCH}_{3}$ & $\mathrm{R}_{3}=\mathrm{H}$ & $\mathrm{R}_{4}=\mathrm{OH}$ & $\mathrm{R}_{s}=\mathrm{H}$ & $\mathrm{R}_{6}=\mathrm{H}$ & $\mathrm{R}_{7}=\mathrm{H}$ & $\mathrm{R}_{8}=\mathrm{H}$ & $\mathrm{R}_{9}=\mathrm{H}$ & $\mathrm{R}_{10}=\mathrm{OH}$ \\
\hline 45 & $\mathrm{R}_{1}=\mathrm{OCH}_{3}$ & $\mathrm{R}_{2}=\mathrm{OCH}_{3}$ & $\mathrm{R}_{3}=\mathrm{H}$ & $\mathrm{R}_{4}=\mathrm{OH}$ & $\mathrm{R}_{s}=\mathrm{H}$ & $\mathrm{R}_{6}=\mathrm{OH}$ & $\mathrm{R}_{7}=\mathrm{H}$ & $\mathrm{R}_{8}=\mathrm{H}$ & $\mathrm{R}_{9}=\mathrm{H}$ & $\mathrm{R}_{10}=\mathrm{OH}$ \\
\hline 46 & $\mathrm{R}_{1}=\mathrm{OCH}_{3}$ & $\mathrm{R}_{2}=\mathrm{OCH}_{3}$ & $\mathrm{R}_{3}=\mathrm{H}$ & $\mathrm{R}_{4}=\mathrm{OH}$ & $\mathrm{R}_{5}=\mathrm{H}$ & $\mathrm{R}_{6}=\mathrm{OCH}_{3}$ & $\mathrm{R}_{7}=\mathrm{H}$ & $\mathrm{R}_{8}=\mathrm{H}$ & $\mathrm{R}_{9}=\mathrm{H}$ & $\mathrm{R}_{10}=\mathrm{OH}$ \\
\hline 47 & $\mathrm{R}_{1}=\mathrm{OCH}_{3}$ & $\mathrm{R}_{2}=\mathrm{OCH}_{3}$ & $\mathrm{R}_{3}=\mathrm{OCH}_{3}$ & $\mathrm{R}_{4}=\mathrm{OCH}_{3}$ & $\mathrm{R}_{5}=\mathrm{H}$ & $\mathrm{R}_{6}=\mathrm{OCH}_{3}$ & $\mathrm{R}_{7}=\mathrm{H}$ & $\mathrm{R}_{8}=\mathrm{H}$ & $\mathrm{R}_{9}=\mathrm{H}$ & $\mathrm{R}_{10}=\mathrm{OH}$ \\
\hline 48 & $\mathrm{R}_{1}=\mathrm{OCH}_{3}$ & $\mathrm{R}_{2}=\mathrm{OCH}_{3}$ & $\mathrm{R}_{3}=\mathrm{OH}$ & $\mathrm{R}_{4}=\mathrm{OCH}_{3}$ & $\mathrm{R}_{\mathrm{s}}=\mathrm{H}$ & $\mathrm{R}_{6}=\mathrm{OCH}_{3}$ & $\mathrm{R}_{7}=\mathrm{H}$ & $\mathrm{R}_{8}=\mathrm{H}$ & $\mathrm{R}_{9}=\mathrm{H}$ & $\mathrm{R}_{10}=\mathrm{OH}$ \\
\hline 49 & $\mathrm{R}_{1}=\mathrm{H}$ & $\mathrm{R}_{2}=\mathrm{OCH}_{3}$ & $\mathrm{R}_{3}=\mathrm{H}$ & $\mathrm{R}_{4}=\mathrm{OCH}_{3}$ & $\mathrm{R}_{5}=\mathrm{H}$ & $\mathrm{R}_{6}=\mathrm{H}$ & $\mathrm{R}_{7}=\mathrm{OCH}_{3}$ & $\mathrm{R}_{8}=\mathrm{OCH}_{3}$ & $\mathrm{R}_{9}=\mathrm{OCH}_{3}$ & $\mathrm{R}_{10}=\mathrm{H}$ \\
\hline 50 & $\mathrm{R}_{1}=\mathrm{H}$ & $\mathrm{R}_{2}=\mathrm{OH}$ & $\mathrm{R}_{3}=\mathrm{H}$ & $\mathrm{R}_{4}=\mathrm{OH}$ & $\mathrm{R}_{5}=\mathrm{OH}$ & $\mathrm{R}_{6}=\mathrm{OH}$ & $\mathrm{R}_{7}=\mathrm{H}$ & $\mathrm{R}_{8}=\mathrm{H}$ & $\mathrm{R}_{9}=\mathrm{H}$ & $\mathrm{R}_{10}=\mathrm{OH}$ \\
\hline 51 & $\mathrm{R}_{1}=\mathrm{H}$ & $\mathrm{R}_{2}=\mathrm{OH}$ & $\mathrm{R}_{3}=\mathrm{H}$ & $\mathrm{R}_{4}=\mathrm{OH}$ & $\mathrm{R}_{5}=\mathrm{OH}$ & $\mathrm{R}_{6}=\mathrm{OCH}_{3}$ & $\mathrm{R}_{7}=\mathrm{H}$ & $\mathrm{R}_{8}=\mathrm{H}$ & $\mathrm{R}_{9}=\mathrm{H}$ & $\mathrm{R}_{10}=\mathrm{OH}$ \\
\hline 52 & $\mathrm{R}_{1}=\mathrm{H}$ & $\mathrm{R}_{2}=\mathrm{OH}$ & $\mathrm{R}_{3}=\mathrm{OGluA}$ & $\mathrm{R}_{4}=\mathrm{OH}$ & $\mathrm{R}_{5}=\mathrm{H}$ & $\mathrm{R}_{6}=\mathrm{H}$ & $\mathrm{R}_{7}=\mathrm{H}$ & $\mathrm{R}_{8}=\mathrm{H}$ & $\mathrm{R}_{9}=\mathrm{H}$ & $\mathrm{R}_{10}=\mathrm{H}$ \\
\hline 53 & $\mathrm{R}_{1}=\mathrm{H}$ & $\mathrm{R}_{2}=\mathrm{OGluA}$ & $\mathrm{R}_{3}=\mathrm{OH}$ & $\mathrm{R}_{4}=\mathrm{OH}$ & $\mathrm{R}_{s}=\mathrm{H}$ & $\mathrm{R}_{6}=\mathrm{H}$ & $\mathrm{R}_{7}=\mathrm{H}$ & $\mathrm{R}_{8}=\mathrm{OH}$ & $\mathrm{R}_{9}=\mathrm{OH}$ & $\mathrm{R}_{10}=\mathrm{H}$ \\
\hline 54 & $\mathrm{R}_{1}=\mathrm{H}$ & $\mathrm{R}_{2}=\mathrm{OGluA}$ & $\mathrm{R}_{3}=\mathrm{H}$ & $\mathrm{R}_{4}=\mathrm{OH}$ & $\mathrm{R}_{5}=\mathrm{H}$ & $\mathrm{R}_{6}=\mathrm{H}$ & $\mathrm{R}_{7}=\mathrm{H}$ & $\mathrm{R}_{8}=\mathrm{OH}$ & $\mathrm{R}_{9}=\mathrm{OH}$ & $\mathrm{R}_{10}=\mathrm{H}$ \\
\hline 55 & $\mathrm{R}_{1}=\mathrm{H}$ & $\mathrm{R}_{2}=\mathrm{ORha}$ & $\mathrm{R}_{3}=\mathrm{H}$ & $\mathrm{R}_{4}=\mathrm{OH}$ & $\mathrm{R}_{\mathrm{s}}=\mathrm{H}$ & $\mathrm{R}_{6}=\mathrm{H}$ & $\mathrm{R}_{7}=\mathrm{H}$ & $\mathrm{R}_{8}=\mathrm{H}$ & $\mathrm{R}_{9}=\mathrm{H}$ & $\mathrm{R}_{10}=\mathrm{H}$ \\
\hline 56 & $\mathrm{R}_{1}=\mathrm{OCH}_{3}$ & $\mathrm{R}_{2}=\mathrm{H}$ & $\mathrm{R}_{3}=\mathrm{H}$ & $\mathrm{R}_{4}=\mathrm{OGluA}$ & $\mathrm{R}_{s}=\mathrm{H}$ & $\mathrm{R}_{6}=\mathrm{H}$ & $\mathrm{R}_{7}=\mathrm{H}$ & $\mathrm{R}_{8}=\mathrm{H}$ & $\mathrm{R}_{9}=\mathrm{H}$ & $\mathrm{R}_{10}=\mathrm{H}$ \\
\hline 57 & $\mathrm{R}_{1}=\mathrm{H}$ & $\mathrm{R}_{2}=\mathrm{OGlc}$ & $\mathrm{R}_{3}=\mathrm{H}$ & $\mathrm{R}_{4}=\mathrm{OH}$ & $\mathrm{R}_{S}=\mathrm{H}$ & $\mathrm{R}_{6}=\mathrm{H}$ & $\mathrm{R}_{7}=\mathrm{H}$ & $\mathrm{R}_{8}=\mathrm{OH}$ & $\mathrm{R}_{9}=\mathrm{H}$ & $\mathrm{R}_{10}=\mathrm{H}$ \\
\hline 58 & $\mathrm{R}_{1}=\mathrm{H}$ & $\mathrm{R}_{2}=\mathrm{OGlc}$ & $\mathrm{R}_{3}=\mathrm{OH}$ & $\mathrm{R}_{4}=\mathrm{OH}$ & $\mathrm{R}_{S}=\mathrm{H}$ & $\mathrm{R}_{6}=\mathrm{H}$ & $\mathrm{R}_{7}=\mathrm{H}$ & $\mathrm{R}_{8}=\mathrm{H}$ & $\mathrm{R}_{9}=\mathrm{H}$ & $\mathrm{R}_{10}=\mathrm{H}$ \\
\hline 59 & $\mathrm{R}_{1}=\mathrm{H}$ & $\mathrm{R}_{2}=\mathrm{OGlc}$ & $\mathrm{R}_{3}=\mathrm{OCH}_{3}$ & $\mathrm{R}_{4}=\mathrm{OH}$ & $\mathrm{R}_{S}=\mathrm{H}$ & $\mathrm{R}_{6}=\mathrm{H}$ & $\mathrm{R}_{7}=\mathrm{H}$ & $\mathrm{R}_{8}=\mathrm{H}$ & $\mathrm{R}_{9}=\mathrm{H}$ & $\mathrm{R}_{10}=\mathrm{H}$ \\
\hline 60 & $\mathrm{R}_{1}=\mathrm{Ara}$ & $\mathrm{R}_{2}=\mathrm{OH}$ & $\mathrm{R}_{3}=\mathrm{Glc}$ & $\mathrm{R}_{4}=\mathrm{OH}$ & $\mathrm{R}_{s}=\mathrm{H}$ & $\mathrm{R}_{6}=\mathrm{H}$ & $\mathrm{R}_{7}=\mathrm{H}$ & $\mathrm{R}_{8}=\mathrm{OH}$ & $\mathrm{R}_{9}=\mathrm{H}$ & $\mathrm{R}_{10}=\mathrm{H}$ \\
\hline 61 & $\mathrm{R}_{1}=\mathrm{OCH}_{3}$ & $\mathrm{R}_{2}=\mathrm{OCH}_{3}$ & $\mathrm{R}_{3}=\mathrm{H}$ & $\mathrm{R}_{4}=\mathrm{OH}$ & $\mathrm{R}_{5}=\mathrm{H}$ & $\mathrm{R}_{6}=\mathrm{OH}$ & $\mathrm{R}_{7}=\mathrm{H}$ & $\mathrm{R}_{8}=\mathrm{H}$ & $\mathrm{R}_{9}=\mathrm{H}$ & $\mathrm{R}_{10}=\mathrm{OGlc}$ \\
\hline 62 & $\mathrm{R}_{1}=\mathrm{OCH}_{3}$ & $\mathrm{R}_{2}=\mathrm{OCH}_{3}$ & $\mathrm{R}_{3}=\mathrm{OCH}_{3}$ & $\mathrm{R}_{4}=\mathrm{OH}$ & $\mathrm{R}_{s}=\mathrm{H}$ & $\mathrm{R}_{6}=\mathrm{OH}$ & $\mathrm{R}_{7}=\mathrm{H}$ & $\mathrm{R}_{8}=\mathrm{H}$ & $\mathrm{R}_{9}=\mathrm{H}$ & $\mathrm{R}_{10}=\mathrm{OGlc}$ \\
\hline 63 & $\mathrm{R}_{1}=\mathrm{H}$ & $\mathrm{R}_{2}=\mathrm{OCH}_{3}$ & $\mathrm{R}_{3}=\mathrm{OCH}_{3}$ & $\mathrm{R}_{4}=\mathrm{OH}$ & $\mathrm{R}_{s}=\mathrm{H}$ & $\mathrm{R}_{6}=\mathrm{OH}$ & $\mathrm{R}_{7}=\mathrm{H}$ & $\mathrm{R}_{8}=\mathrm{H}$ & $\mathrm{R}_{9}=\mathrm{H}$ & $\mathrm{R}_{10}=\mathrm{OGlc}$ \\
\hline 64 & $\mathrm{R}_{1}=\mathrm{H}$ & $\mathrm{R}_{2}=\mathrm{OH}$ & $\mathrm{R}_{3}=\mathrm{H}$ & $\mathrm{R}_{4}=\mathrm{OH}$ & $\mathrm{R}_{5}=\mathrm{H}$ & $\mathrm{R}_{6}=\mathrm{OH}$ & $\mathrm{R}_{7}=\mathrm{H}$ & $\mathrm{R}_{8}=\mathrm{H}$ & $\mathrm{R}_{9}=\mathrm{H}$ & $\mathrm{R}_{10}=\mathrm{OGlc}$ \\
\hline 65 & $\mathrm{R}_{1}=\mathrm{OCH}_{3}$ & $\mathrm{R}_{2}=\mathrm{OH}$ & $\mathrm{R}_{3}=\mathrm{H}$ & $\mathrm{R}_{4}=\mathrm{OH}$ & $\mathrm{R}_{\mathrm{s}}=\mathrm{H}$ & $\mathrm{R}_{6}=\mathrm{OCH}_{3}$ & $\mathrm{R}_{7}=\mathrm{OH}$ & $\mathrm{R}_{8}=\mathrm{H}$ & $\mathrm{R}_{9}=\mathrm{H}$ & $\mathrm{R}_{10}=\mathrm{OGlc}$ \\
\hline 66 & $\mathrm{R}_{1}=\mathrm{OCH}_{3}$ & $\mathrm{R}_{2}=\mathrm{OH}$ & $\mathrm{R}_{3}=\mathrm{H}$ & $\mathrm{R}_{4}=\mathrm{OGlc}$ & $\mathrm{R}_{5}=\mathrm{H}$ & $\mathrm{R}_{6}=\mathrm{H}$ & $\mathrm{R}_{7}=\mathrm{H}$ & $\mathrm{R}_{8}=\mathrm{H}$ & $\mathrm{R}_{9}=\mathrm{H}$ & $\mathrm{R}_{10}=\mathrm{H}$ \\
\hline 67 & $\mathrm{R}_{1}=\mathrm{H}$ & $\mathrm{R}_{2}=\mathrm{OH}$ & $\mathrm{R}_{3}=\mathrm{H}$ & $\mathrm{R}_{4}=\mathrm{OH}$ & $\mathrm{R}_{5}=\mathrm{OH}$ & $\mathrm{R}_{6}=\mathrm{OH}$ & $\mathrm{R}_{7}=\mathrm{H}$ & $\mathrm{R}_{8}=\mathrm{H}$ & $\mathrm{R}_{9}=\mathrm{H}$ & $\mathrm{R}_{10}=\mathrm{OGlc}$ \\
\hline 68 & $\mathrm{R}_{1}=\mathrm{H}$ & $\mathrm{R}_{2}=\mathrm{OH}$ & $\mathrm{R}_{3}=\mathrm{H}$ & $\mathrm{R}_{4}=\mathrm{OH}$ & $\mathrm{R}_{5}=\mathrm{OGlc}$ & $\mathrm{R}_{6}=\mathrm{H}$ & $\mathrm{R}_{7}=\mathrm{H}$ & $\mathrm{R}_{8}=\mathrm{OH}$ & $\mathrm{R}_{9}=\mathrm{H}$ & $\mathrm{R}_{10}=\mathrm{H}$ \\
\hline 69 & $\mathrm{R}_{1}=\mathrm{H}$ & $\mathrm{R}_{2}=$ OGluA & $\mathrm{R}_{3}=\mathrm{H}$ & $\mathrm{R}_{4}=\mathrm{OH}$ & $\mathrm{R}_{5}=\mathrm{H}$ & $\mathrm{R}_{6}=\mathrm{H}$ & $\mathrm{R}_{7}=\mathrm{H}$ & $\mathrm{R}_{8}=\mathrm{H}$ & $\mathrm{R}_{9}=\mathrm{H}$ & $\mathrm{R}_{10}=\mathrm{H}$ \\
\hline 70 & $\mathrm{R}_{1}=\mathrm{H}$ & $\mathrm{R}_{2}=$ OGluA & $\mathrm{R}_{3}=\mathrm{OH}$ & $\mathrm{R}_{4}=\mathrm{OH}$ & $\mathrm{R}_{5}=\mathrm{H}$ & $\mathrm{R}_{6}=\mathrm{H}$ & $\mathrm{R}_{7}=\mathrm{H}$ & $\mathrm{R}_{8}=\mathrm{H}$ & $\mathrm{R}_{9}=\mathrm{H}$ & $\mathrm{R}_{10}=\mathrm{H}$ \\
\hline 71 & $\mathrm{R}_{1}=\mathrm{H}$ & $\mathrm{R}_{2}=$ OGluA & $\mathrm{R}_{3}=\mathrm{OCH}_{3}$ & $\mathrm{R}_{4}=\mathrm{OH}$ & $\mathrm{R}_{5}=\mathrm{H}$ & $\mathrm{R}_{6}=\mathrm{H}$ & $\mathrm{R}_{7}=\mathrm{H}$ & $\mathrm{R}_{8}=\mathrm{H}$ & $\mathrm{R}_{9}=\mathrm{H}$ & $\mathrm{R}_{10}=\mathrm{OH}$ \\
\hline 72 & $\mathrm{R}_{1}=\mathrm{OCH}_{3}$ & $\mathrm{R}_{2}=\mathrm{OGluA}$ & $\mathrm{R}_{3}=\mathrm{H}$ & $\mathrm{R}_{4}=\mathrm{OH}$ & $\mathrm{R}_{\mathrm{s}}=\mathrm{H}$ & $\mathrm{R}_{6}=\mathrm{H}$ & $\mathrm{R}_{7}=\mathrm{H}$ & $\mathrm{R}_{8}=\mathrm{H}$ & $\mathrm{R}_{9}=\mathrm{H}$ & $\mathrm{R}_{10}=\mathrm{H}$ \\
\hline 73 & $\mathrm{R}_{1}=\mathrm{H}$ & $\mathrm{R}_{2}=\mathrm{OGluA}$ & $\mathrm{R}_{3}=\mathrm{OCH}_{3}$ & $\mathrm{R}_{4}=\mathrm{OH}$ & $\mathrm{R}_{5}=\mathrm{H}$ & $\mathrm{R}_{6}=\mathrm{H}$ & $\mathrm{R}_{7}=\mathrm{H}$ & $\mathrm{R}_{8}=\mathrm{H}$ & $\mathrm{R}_{9}=\mathrm{H}$ & $\mathrm{R}_{10}=\mathrm{H}$ \\
\hline $\begin{array}{l}74 \\
\text { Fig. }\end{array}$ & $\begin{array}{l}\mathrm{R}_{1}=\mathrm{OH} \\
.1 \text { continue }\end{array}$ & $\mathrm{R}_{2}=\mathrm{OGluA}$ & $\mathrm{R}_{3}=\mathrm{H}$ & $\mathrm{R}_{4}=\mathrm{OH}$ & $\mathrm{R}_{5}=\mathrm{H}$ & $\mathrm{R}_{6}=\mathrm{H}$ & $\mathrm{R}_{7}=\mathrm{H}$ & $\mathrm{R}_{8}=\mathrm{H}$ & $\mathrm{R}_{9}=\mathrm{H}$ & $\mathrm{R}_{10}=\mathrm{H}$ \\
\hline
\end{tabular}

can significantly inhibit the up-regulation of IL-1 $\beta$ and IL-8 level caused by Propionibacterium acnes via inactivation of the MAPK and NF- $k B$ signaling pathways. The main effective components are wogonin and wogonoside [65]. Baicalin had a dose-dependent inhibitory effect on the expression of LasA protease, LasB elastase, pyocyanin, rhamnolipid, functional and exotoxin A caused by
Pseudomonas aeruginosa through changing gene and protein expression [66]. Therefore, SB not only decreased the reproduction of bacteria but also stimulated TH1 induced immune response to accelerate bacterial clearance. Baicalin aluminum complexes can change the whole structure and composition of intestinal microbiome of diarrhea piglets, so as to alleviate diarrhea 


\begin{tabular}{|c|c|c|c|c|c|c|c|c|c|c|}
\hline 75 & $\mathrm{R}_{1}=\mathrm{OGluA}$ & $\mathrm{R}_{2}=\mathrm{OCH}_{3}$ & $\mathrm{R}_{3}=\mathrm{H}$ & $\mathrm{R}_{4}=\mathrm{OCH}_{3}$ & $\mathrm{R}_{5}=\mathrm{H}$ & $\mathrm{R}_{6}=\mathrm{H}$ & $\mathrm{R}_{7}=\mathrm{H}$ & $\mathrm{R}_{8}=\mathrm{OCH}_{3}$ & $\mathrm{R}_{9}=\mathrm{H}$ & $\mathrm{R}_{10}=\mathrm{H}$ \\
\hline 76 & $\mathrm{R}_{1}=\mathrm{OCH}_{3}$ & $\mathrm{R}_{2}=\mathrm{OCH}_{3}$ & $\mathrm{R}_{3}=\mathrm{H}$ & $\mathrm{R}_{4}=\mathrm{OH}$ & $\mathrm{R}_{5}=\mathrm{H}$ & $\mathrm{R}_{6}=\mathrm{OCH}_{3}$ & $\mathrm{R}_{7}=\mathrm{H}$ & $\mathrm{R}_{8}=\mathrm{H}$ & $\mathrm{R}_{9}=\mathrm{H}$ & $\mathrm{R}_{10}=\mathrm{OGluA}$ \\
\hline 77 & $\mathrm{R}_{1}=\mathrm{H}$ & $\mathrm{R}_{2}=\mathrm{OGluA}$ & $\mathrm{R}_{3}=\mathrm{OH}$ & $\mathrm{R}_{4}=\mathrm{OH}$ & $\mathrm{R}_{5}=\mathrm{H}$ & $\mathrm{R}_{6}=\mathrm{H}$ & $\mathrm{R}_{7}=\mathrm{H}$ & $\mathrm{R}_{8}=\mathrm{OH}$ & $\mathrm{R}_{9}=\mathrm{H}$ & $\mathrm{R}_{10}=\mathrm{H}$ \\
\hline 78 & $\mathrm{R}_{1}=\mathrm{H}$ & $\mathrm{R}_{2}=\mathrm{OGluA}$ & $\mathrm{R}_{3}=\mathrm{H}$ & $\mathrm{R}_{4}=\mathrm{OH}$ & $\mathrm{R}_{5}=\mathrm{H}$ & $\mathrm{R}_{6}=\mathrm{H}$ & $\mathrm{R}_{7}=\mathrm{H}$ & $\mathrm{R}_{8}=\mathrm{OH}$ & $\mathrm{R}_{9}=\mathrm{H}$ & $\mathrm{R}_{10}=\mathrm{H}$ \\
\hline 79 & $\mathrm{R}_{1}=\mathrm{H}$ & $\mathrm{R}_{2}=\mathrm{OGluA}$ & $\mathrm{R}_{3}=\mathrm{OCH}_{3}$ & $\mathrm{R}_{4}=\mathrm{OH}$ & $\mathrm{R}_{5}=\mathrm{OH}$ & $\mathrm{R}_{6}=\mathrm{H}$ & $\mathrm{R}_{7}=\mathrm{OH}$ & $\mathrm{R}_{8}=\mathrm{OH}$ & $\mathrm{R}_{9}=\mathrm{H}$ & $\mathrm{R}_{10}=\mathrm{H}$ \\
\hline 80 & $\mathrm{R}_{1}=\mathrm{Glc}$ & $\mathrm{R}_{2}=\mathrm{OH}$ & $\mathrm{R}_{3}=\mathrm{H}$ & $\mathrm{R}_{4}=\mathrm{OH}$ & $\mathrm{R}_{5}=\mathrm{H}$ & $\mathrm{R}_{6}=\mathrm{H}$ & $\mathrm{R}_{7}=\mathrm{H}$ & $\mathrm{R}_{8}=\mathrm{H}$ & $\mathrm{R}_{9}=\mathrm{H}$ & $\mathrm{R}_{10}=\mathrm{H}$ \\
\hline 81 & $\mathrm{R}_{1}=\mathrm{H}$ & $\mathrm{R}_{2}=\mathrm{OH}$ & $\mathrm{R}_{3}=\mathrm{Glc}$ & $\mathrm{R}_{4}=\mathrm{OH}$ & $\mathrm{R}_{5}=\mathrm{H}$ & $\mathrm{R}_{6}=\mathrm{H}$ & $\mathrm{R}_{7}=\mathrm{H}$ & $\mathrm{R}_{8}=\mathrm{H}$ & $\mathrm{R}_{9}=\mathrm{H}$ & $\mathrm{R}_{10}=\mathrm{H}$ \\
\hline 82 & $\mathrm{R}_{1}=\operatorname{Ara}-\mathrm{p}(\alpha, \mathrm{L})$ & $\mathrm{R}_{2}=\mathrm{OH}$ & $\mathrm{R}_{3}=\mathrm{Glc}$ & $\mathrm{R}_{4}=\mathrm{OH}$ & $\mathrm{R}_{5}=\mathrm{H}$ & $\mathrm{R}_{6}=\mathrm{H}$ & $\mathrm{R}_{7}=\mathrm{H}$ & $\mathrm{R}_{8}=\mathrm{H}$ & $\mathrm{R}_{9}=\mathrm{H}$ & $\mathrm{R}_{10}=\mathrm{H}$ \\
\hline 83 & $\mathrm{R}_{1}=\mathrm{Glc}$ & $\mathrm{R}_{2}=\mathrm{OH}$ & $\mathrm{R}_{3}=\operatorname{Ara}-\mathrm{p}(\alpha, \mathrm{L})$ & $\mathrm{R}_{4}=\mathrm{OH}$ & $\mathrm{R}_{5}=\mathrm{H}$ & $\mathrm{R}_{6}=\mathrm{H}$ & $\mathrm{R}_{7}=\mathrm{H}$ & $\mathrm{R}_{8}=\mathrm{H}$ & $\mathrm{R}_{9}=\mathrm{H}$ & $\mathrm{R}_{10}=\mathrm{H}$ \\
\hline 84 & $\mathrm{R}_{\mathrm{l}}=\mathrm{Glc}$ & $\mathrm{R}_{2}=\mathrm{OH}$ & $\mathrm{R}_{3}=\operatorname{Ara}-\mathrm{p}(\beta, \mathrm{L})$ & $\mathrm{R}_{4}=\mathrm{OH}$ & $\mathrm{R}_{5}=\mathrm{H}$ & $\mathrm{R}_{6}=\mathrm{H}$ & $\mathrm{R}_{7}=\mathrm{H}$ & $\mathrm{R}_{8}=\mathrm{H}$ & $\mathrm{R}_{9}=\mathrm{H}$ & $\mathrm{R}_{10}=\mathrm{H}$ \\
\hline 85 & $\mathrm{R}_{1}=\operatorname{Ara}-\mathrm{p}(\beta, \mathrm{L})$ & $\mathrm{R}_{2}=\mathrm{OH}$ & $\mathrm{R}_{3}=\mathrm{Glc}$ & $\mathrm{R}_{4}=\mathrm{OH}$ & $\mathrm{R}_{5}=\mathrm{H}$ & $\mathrm{R}_{6}=\mathrm{H}$ & $\mathrm{R}_{7}=\mathrm{H}$ & $\mathrm{R}_{8}=\mathrm{H}$ & $\mathrm{R}_{9}=\mathrm{H}$ & $\mathrm{R}_{10}=\mathrm{H}$ \\
\hline 86 & $\mathrm{R}_{1}=\mathrm{Glc}$ & $\mathrm{R}_{2}=\mathrm{OH}$ & $\mathrm{R}_{3}=\operatorname{Ara}-\mathrm{f}(\beta)$ & $\mathrm{R}_{4}=\mathrm{OH}$ & $\mathrm{R}_{5}=\mathrm{H}$ & $\mathrm{R}_{6}=\mathrm{H}$ & $\mathrm{R}_{7}=\mathrm{H}$ & $\mathrm{R}_{8}=\mathrm{H}$ & $\mathrm{R}_{9}=\mathrm{H}$ & $\mathrm{R}_{10}=\mathrm{H}$ \\
\hline 87 & $\mathrm{R}_{1}=\operatorname{Ara}-\mathrm{f}(\beta)$ & $\mathrm{R}_{2}=\mathrm{OH}$ & $\mathrm{R}_{3}=\mathrm{Glc}$ & $\mathrm{R}_{4}=\mathrm{OH}$ & $\mathrm{R}_{5}=\mathrm{H}$ & $\mathrm{R}_{6}=\mathrm{H}$ & $\mathrm{R}_{7}=\mathrm{H}$ & $\mathrm{R}_{8}=\mathrm{H}$ & $\mathrm{R}_{9}=\mathrm{H}$ & $\mathrm{R}_{10}=\mathrm{H}$ \\
\hline 88 & $\mathrm{R}_{1}=\mathrm{Glc}$ & $\mathrm{R}_{2}=\mathrm{OH}$ & $\mathrm{R}_{3}=\mathrm{H}$ & $\mathrm{R}_{4}=\mathrm{OH}$ & $\mathrm{R}_{5}=\operatorname{Ara}-\mathrm{p}(\alpha)$ & $\mathrm{R}_{6}=\mathrm{H}$ & $\mathrm{R}_{7}=\mathrm{H}$ & $\mathrm{R}_{8}=\mathrm{H}$ & $\mathrm{R}_{9}=\mathrm{H}$ & $\mathrm{R}_{10}=\mathrm{H}$ \\
\hline 89 & $\mathrm{R}_{1}=\mathrm{Glc}$ & $\mathrm{R}_{2}=\mathrm{OH}$ & $\mathrm{R}_{3}=\operatorname{Ara}-\mathrm{p}(\alpha, \mathrm{L})$ & $\mathrm{R}_{4}=\mathrm{OH}$ & $\mathrm{R}_{5}=\mathrm{H}$ & $\mathrm{R}_{6}=\mathrm{H}$ & $\mathrm{R}_{7}=\mathrm{H}$ & $\mathrm{R}_{8}=\mathrm{OH}$ & $\mathrm{R}_{9}=\mathrm{H}$ & $\mathrm{R}_{10}=\mathrm{H}$ \\
\hline 90 & $\mathrm{R}_{1}=\mathrm{OCH}_{3}$ & $\mathrm{R}_{2}=\mathrm{OH}$ & $\mathrm{R}_{3}=\mathrm{OCH}_{3}$ & $\mathrm{R}_{4}=\mathrm{OH}$ & $\mathrm{R}_{5}=\mathrm{H}$ & $\mathrm{R}_{6}=\mathrm{H}$ & $\mathrm{R}_{7}=\mathrm{OH}$ & $\mathrm{R}_{8}=\mathrm{H}$ & $\mathrm{R}_{9}=\mathrm{H}$ & $\mathrm{R}_{10}=\mathrm{OGlc}$ \\
\hline 91 & $\mathrm{R}_{1}=\mathrm{H}$ & $\mathrm{R}_{2}=\mathrm{OGluA}$ & $\mathrm{R}_{3}=\mathrm{H}$ & $\mathrm{R}_{4}=\mathrm{OH}$ & $\mathrm{R}_{5}=\mathrm{OH}$ & & $\mathrm{R}_{7}=\mathrm{H}$ & $\mathrm{R}_{8}=\mathrm{OH}$ & $\mathrm{R}_{9}=\mathrm{OH}$ & $\mathrm{R}_{10}=\mathrm{H}$ \\
\hline 92 & $\mathrm{R}_{1}=\mathrm{H}$ & $\mathrm{R}_{2}=\mathrm{OH}$ & $\mathrm{R}_{3}=\mathrm{H}$ & $\mathrm{R}_{4}=\mathrm{OH}$ & $\mathrm{R}_{5}=\mathrm{H}$ & $\mathrm{R}_{6}=\mathrm{H}$ & $\mathrm{R}_{7}=\mathrm{H}$ & $\mathrm{R}_{8}=\mathrm{OH}$ & $\mathrm{R}_{9}=\mathrm{H}$ & $\mathrm{R}_{10}=\mathrm{H}$ \\
\hline 93 & $\mathrm{R}_{1}=\mathrm{H}$ & $\mathrm{R}_{2}=\mathrm{OH}$ & $\mathrm{R}_{3}=\mathrm{H}$ & $\mathrm{R}_{4}=\mathrm{OH}$ & $\mathrm{R}_{5}=\mathrm{H}$ & $\mathrm{R}_{6}=\mathrm{H}$ & $\mathrm{R}_{7}=\mathrm{H}$ & $\mathrm{R}_{8}=\mathrm{H}$ & $\mathrm{R}_{9}=\mathrm{H}$ & $\mathrm{R}_{10}=\mathrm{H}$ \\
\hline 94 & $\mathrm{R}_{1}=\mathrm{OH}$ & $\mathrm{R}_{2}=\mathrm{OH}$ & $\mathrm{R}_{3}=\mathrm{H}$ & $\mathrm{R}_{4}=\mathrm{OH}$ & $\mathrm{R}_{5}=\mathrm{H}$ & $\mathrm{R}_{6}=\mathrm{H}$ & $\mathrm{R}_{7}=\mathrm{H}$ & $\mathrm{R}_{8}=\mathrm{OH}$ & $\mathrm{R}_{9}=\mathrm{H}$ & $\mathrm{R}_{10}=\mathrm{H}$ \\
\hline 95 & $\mathrm{R}_{1}=\mathrm{H}$ & $\mathrm{R}_{2}=\mathrm{OH}$ & $\mathrm{R}_{3}=\mathrm{OH}$ & $\mathrm{R}_{4}=\mathrm{OH}$ & $\mathrm{R}_{5}=\mathrm{H}$ & $\mathrm{R}_{6}=\mathrm{H}$ & $\mathrm{R}_{7}=\mathrm{H}$ & $\mathrm{R}_{8}=\mathrm{OH}$ & $\mathrm{R}_{9}=\mathrm{H}$ & $\mathrm{R}_{10}=\mathrm{H}$ \\
\hline 96 & $\mathrm{R}_{1}=\mathrm{H}$ & $\mathrm{R}_{2}=\mathrm{OH}$ & $\mathrm{R}_{3}=\mathrm{OCH}_{3}$ & $\mathrm{R}_{4}=\mathrm{OH}$ & $\mathrm{R}_{5}=\mathrm{H}$ & $\mathrm{R}_{6}=\mathrm{H}$ & $\mathrm{R}_{7}=\mathrm{H}$ & $\mathrm{R}_{8}=\mathrm{OH}$ & $\mathrm{R}_{9}=\mathrm{H}$ & $\mathrm{R}_{10}=\mathrm{H}$ \\
\hline 97 & $\mathrm{R}_{1}=\mathrm{H}$ & $\mathrm{R}_{2}=\mathrm{OH}$ & $\mathrm{R}_{3}=\mathrm{H}$ & $\mathrm{R}_{4}=\mathrm{OH}$ & $\mathrm{R}_{5}=\mathrm{H}$ & $\mathrm{R}_{6}=\mathrm{H}$ & $\mathrm{R}_{7}=\mathrm{H}$ & $\mathrm{R}_{8}=\mathrm{OH}$ & $\mathrm{R}_{9}=\mathrm{OH}$ & $\mathrm{R}_{10}=\mathrm{H}$ \\
\hline 98 & $\mathrm{R}_{1}=\mathrm{H}$ & $\mathrm{R}_{2}=\mathrm{OCH}_{3}$ & $\mathrm{R}_{3}=\mathrm{H}$ & $\mathrm{R}_{4}=\mathrm{OH}$ & $\mathrm{R}_{5}=\mathrm{H}$ & $\mathrm{R}_{6}=\mathrm{H}$ & $\mathrm{R}_{7}=\mathrm{H}$ & $\mathrm{R}_{8}=\mathrm{OH}$ & $\mathrm{R}_{9}=\mathrm{H}$ & $\mathrm{R}_{10}=\mathrm{H}$ \\
\hline 99 & $\mathrm{R}_{1}=\mathrm{H}$ & $\mathrm{R}_{2}=\mathrm{OH}$ & $\mathrm{R}_{3}=\mathrm{OCH}_{3}$ & $\mathrm{R}_{4}=\mathrm{OH}$ & $\mathrm{R}_{5}=\mathrm{H}$ & $\mathrm{R}_{6}=\mathrm{H}$ & $\mathrm{R}_{7}=\mathrm{H}$ & $\mathrm{R}_{8}=\mathrm{H}$ & $\mathrm{R}_{9}=\mathrm{H}$ & $\mathrm{R}_{10}=\mathrm{H}$ \\
\hline 100 & $\mathrm{R}_{1}=\mathrm{H}$ & $\mathrm{R}_{2}=\mathrm{OH}$ & $\mathrm{R}_{3}=\mathrm{H}$ & $\mathrm{R}_{4}=\mathrm{OCH}_{3}$ & $\mathrm{R}_{5}=\mathrm{H}$ & $\mathrm{R}_{6}=\mathrm{H}$ & $\mathrm{R}_{7}=\mathrm{H}$ & $\mathrm{R}_{8}=\mathrm{H}$ & $\mathrm{R}_{9}=\mathrm{H}$ & $\mathrm{R}_{10}=\mathrm{H}$ \\
\hline 101 & $\mathrm{R}_{1}=\mathrm{H}$ & $\mathrm{R}_{2}=\mathrm{OH}$ & $\mathrm{R}_{3}=\mathrm{H}$ & $\mathrm{R}_{4}=\mathrm{OH}$ & $\mathrm{R}_{S}=\mathrm{H}$ & $\mathrm{R}_{6}=\mathrm{H}$ & $\mathrm{R}_{7}=\mathrm{OH}$ & $\mathrm{R}_{8}=\mathrm{H}$ & $\mathrm{R}_{9}=\mathrm{H}$ & $\mathrm{R}_{10}=\mathrm{OH}$ \\
\hline 102 & $\mathrm{R}_{1}=\mathrm{H}$ & $\mathrm{R}_{2}=\mathrm{OH}$ & $\mathrm{R}_{3}=\mathrm{H}$ & $\mathrm{R}_{4}=\mathrm{OH}$ & $\mathrm{R}_{5}=\mathrm{H}$ & $\mathrm{R}_{6}=\mathrm{OH}$ & $\mathrm{R}_{7}=\mathrm{H}$ & $\mathrm{R}_{8}=\mathrm{H}$ & $\mathrm{R}_{9}=\mathrm{H}$ & $\mathrm{R}_{10}=\mathrm{OH}$ \\
\hline 103 & $\mathrm{R}_{1}=\mathrm{H}$ & $\mathrm{R}_{2}=\mathrm{OH}$ & $\mathrm{R}_{3}=\mathrm{H}$ & $\mathrm{R}_{4}=\mathrm{OCH}_{3}$ & $\mathrm{R}_{5}=\mathrm{H}$ & $\mathrm{R}_{6}=\mathrm{OH}$ & $\mathrm{R}_{7}=\mathrm{H}$ & $\mathrm{R}_{8}=\mathrm{H}$ & $\mathrm{R}_{9}=\mathrm{H}$ & $\mathrm{R}_{10}=\mathrm{OH}$ \\
\hline 104 & $\mathrm{R}_{1}=\mathrm{H}$ & $\mathrm{R}_{2}=\mathrm{OH}$ & $\mathrm{R}_{3}=\mathrm{H}$ & $\mathrm{R}_{4}=\mathrm{OH}$ & $\mathrm{R}_{5}=\mathrm{OH}$ & $\mathrm{R}_{6}=\mathrm{OH}$ & $\mathrm{R}_{7}=\mathrm{H}$ & $\mathrm{R}_{8}=\mathrm{H}$ & $\mathrm{R}_{9}=\mathrm{H}$ & $\mathrm{R}_{10}=\mathrm{OH}$ \\
\hline $\begin{array}{l}105 \\
\text { Fig. }\end{array}$ & $\begin{array}{l}\mathrm{R}_{1}=\text { Ara- } \mathrm{p} \\
1 \text { continued }\end{array}$ & $\mathrm{R}_{2}=\mathrm{OH}$ & $\mathrm{R}_{3}=\mathrm{Glc}$ & $\mathrm{R}_{4}=\mathrm{OH}$ & $\mathrm{R}_{5}=\mathrm{OH}$ & $\mathrm{R}_{6}=\mathrm{H}$ & $\mathrm{R}_{7}=\mathrm{H}$ & $\mathrm{R}_{8}=\mathrm{H}$ & $\mathrm{R}_{9}=\mathrm{H}$ & $\mathrm{R}_{10}=\mathrm{H}$ \\
\hline
\end{tabular}

symptoms. However, its further antibacterial mechanism and regulation of microorganisms have not been clearly described [67]. At present, baicalein aluminum capsule has been used to treat diarrhea.

\section{Antiviral}

$\mathrm{T}$ cell infiltration and cytotoxic T-cell-mediated tissue damage have been identified as key factors in RSV disease. Baicalin can reduce $\mathrm{T}$ lymphocyte infiltration and pro-inflammatory factor gene expression to play the effect of antiviral [68]. IAV can cause acute lung injury, the specific manifestations were lung index and abnormal lung tissue lesions. SBE significantly improved these lesions [69] by increasing the activity of HA and NA. And the level of inflammatory factors in lung tissue were regulated to inhibit the inflammatory response. SBE can inhibit the propagation of dengue virus, and baicalein is the main active ingredient in the extract [70]. Oroxylin A significantly protects Vero cells from CVB3-induced cell death in vitro, and can improve the symptoms which reduced body weight and blood glucose levels in vivo [71]. $7 \mu \mathrm{M}$ baicalin can 


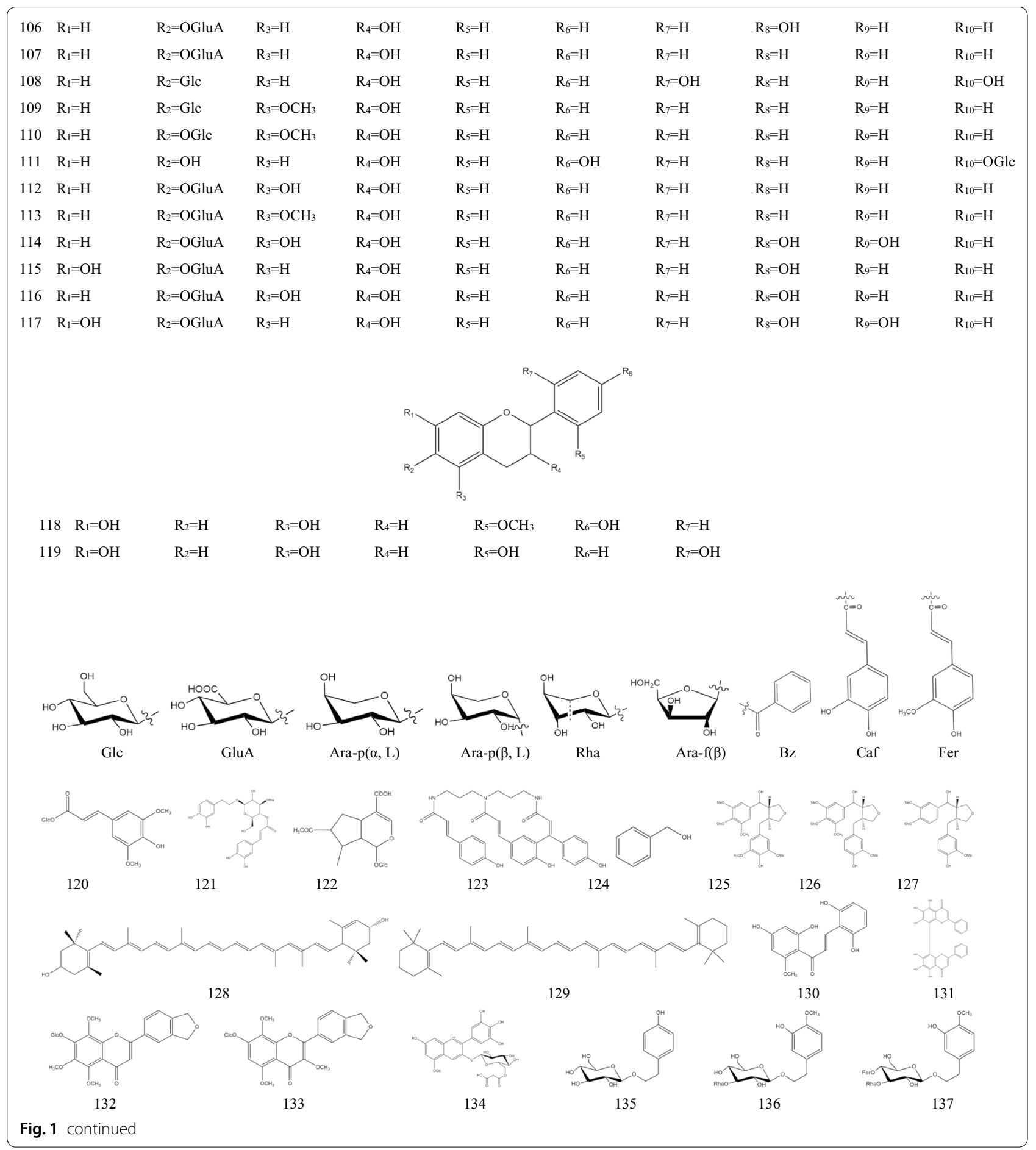

produce directly virus-killing activity against CHIKV in vitro [72], the levels of important protein markers for autophagy and apoptosis were reduced.

\section{Antioxidant}

Wogonin 7-O- $\beta$-D-ethylglucuronide, wogonoside and baicalein 7-O- $\beta$-D-ethylglucuronide have antioxidant 


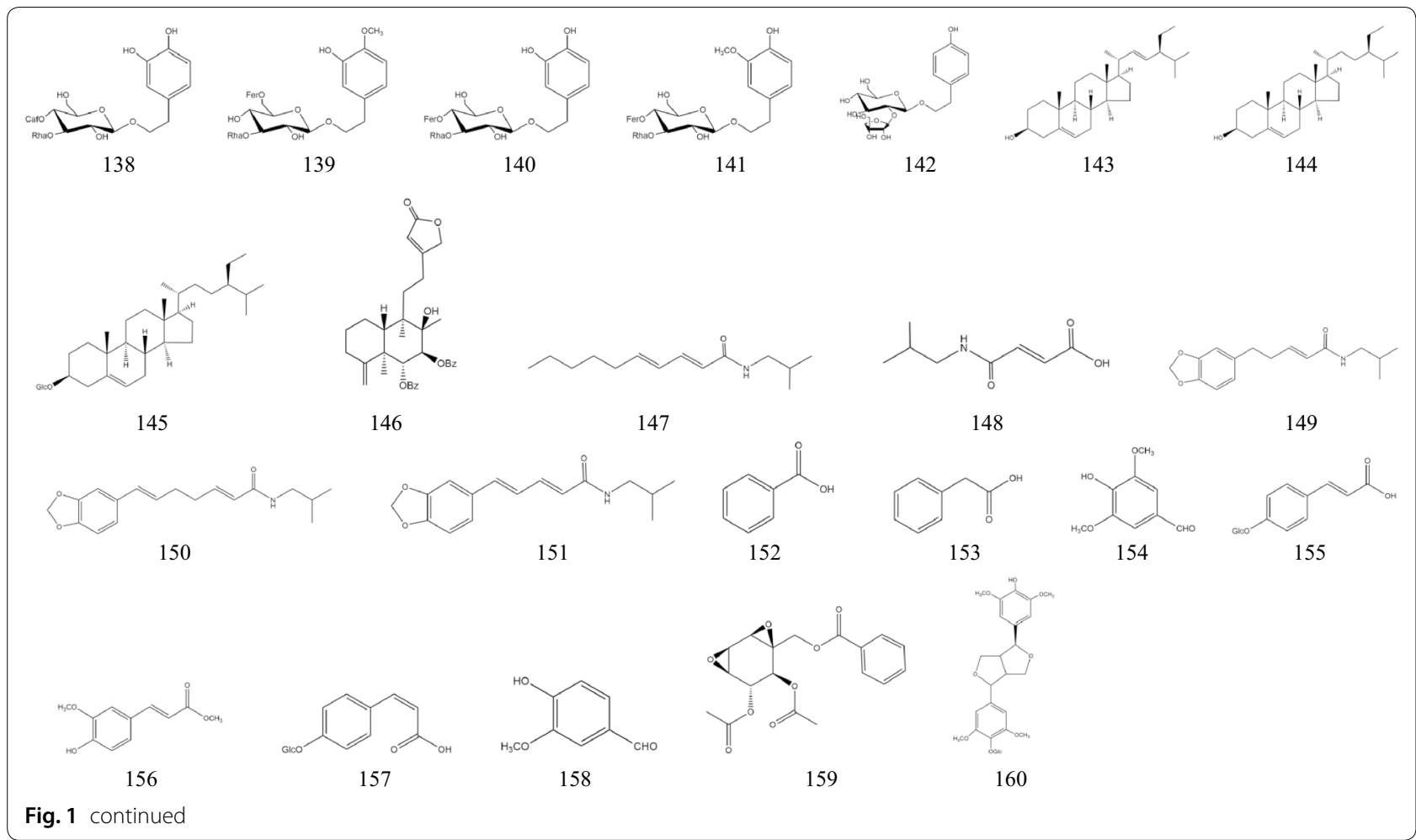

activity [73]. They can inhibit $\mathrm{FeSO}_{4}$-Cys-induced lipid peroxidation of liver homogenate, and showed strong cytoprotective effect on $\mathrm{H}_{2} \mathrm{O}_{2}$-induced oxidative damage of human umbilical vein endothelial cells. SBE was found to effectively attenuate comet tail formation and inhibit histone $\gamma \mathrm{H} 2 \mathrm{AX}$ phosphorylation induced by $\mathrm{H}_{2} \mathrm{O}_{2}$ [74]. It can also restore the loss of mitochondrial membrane potential by $\mathrm{H}_{2} \mathrm{O}_{2}$ and has the activity of scavenging ROS production in cells. More importantly, SBE blocks the oxidative stress by activating Nrf2/HO- 1 signaling pathway to inhibit DNA and cell damage and apoptosis induced by $\mathrm{H}_{2} \mathrm{O}_{2}$. The antioxidant activities of the mixture of polyphenols from four tissues of SB are remarkable [39]. Root has the highest antioxidant activity, followed by leaf, stem and flower. A baicalin/Pluronic F127 hydrogel has excellent cell compatibility and resistance to oxidative stress caused by reactive oxygen species, and accelerate wound healing [75]. In summary, SB has a strong antioxidant effect, and can indirectly exert a cell protective effect through the antioxidant activity.

\section{Cardiovascular effect}

Cerebral ischemia will have neuropathological abnormal symptoms such as neuron loss or swelling, $\mathrm{Na}^{+}-\mathrm{K}^{+}$-ATPase, $\mathrm{Ca}^{2+}$-ATPase and the activity of SOD are significantly reduced, and the level of MDA is increased. The flavonoids in SB $(35-140 \mathrm{mg} / \mathrm{kg})$ have a significant improvement on the above abnormal conditions, indicating that the flavonoids in SB have a significant therapeutic effect on cerebral ischemia-reperfusion [76]. LysoPC is a membrane phospholipid metabolite that accumulates in ischemic myocardium and plays an important role in the occurrence of ventricular arrhythmias in myocardial dysfunction. Baicalein can protects $\mathrm{H} 9 \mathrm{c} 2$ embryonic cardiomyocytes from hemolysin-induced cytotoxicity [77]. It prevented lysoPC-induced cardiomyocyte death, ROS production and the rise of $\mathrm{Ca}^{2+}$ concentration in H9c2 cardiomyocyte through the MAPK pathway. In addition, the ratio of $\mathrm{Bcl}-2 / \mathrm{Bax}$ was increased and the expression of cytochrome c, caspase-3, caspase-9 were decreased.

Baicalin can play an anti-hypertensive effect by improving the state of intestinal injury [78]. Intestinal barrier damage plays an important role in the pathogenesis of hypertension. Baicalin can reduce the proximal colonic lesions, intestinal permeability and release levels of related inflammatory factors to achieve anti-hypertensive effect. In addition, SCFA-producing bacteria were induced to increase. Baicalin, baicalein and wogonoside in Sanhuang decoction have vasodilating effects in vitro and anti-hypertensive effect in vivo. It is speculated that these components play roles by activating the NO/cGMP pathway, and the BKCa channel and DAG/PKC/CPI-17 pathway are also involved [79]. Furthermore, baicalin 
<smiles>N[C@@H](Cc1ccccc1)C(=O)O</smiles>

Phenylalanine<smiles>CC(C)(C)C=CC(=O)O</smiles>

Cinnamic acid<smiles>O=C(O)C=Cc1ccccc1</smiles>

Cinnamoyl-CoA

CHS-2<smiles>O=C(/C=C/c1ccccc1)c1c(O)cc(O)cc1O</smiles>

Pinocembrin chalcone<smiles>CC1(C)Oc2cc(O)cc(O)c2C(=O)CC1c1ccccc1</smiles>

Pinocembrin<smiles>CCCCCCCCCC=CC(=O)O</smiles>

4-Coumarate<smiles>O=C(Cl)/C=C/c1cccc(O)c1</smiles>

4-Coumarate-CoA<smiles>CCCCc1cc(O)ccc1/C=C/C(=O)c1c(O)cc(O)cc1O</smiles>

Naringenin chalcone<smiles>Cn1c(-c2ccccc2)cc(=O)c2c(O)cc(O)cc21</smiles>

Chrysin<smiles>O=c1cc(-c2ccccc2)oc2cc(O)c(O)c(O)c12</smiles>

Baicalein

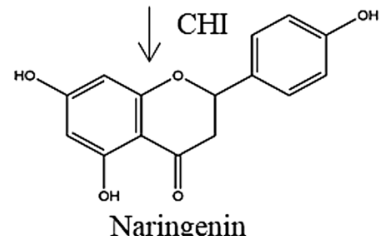<smiles>O=c1cc(-c2ccccc2)oc2c(O)c(O)cc(O)c12</smiles>

Norwogonin<smiles>COc1c(O)cc(O)c2c(=O)cc(-c3ccccc3)oc12</smiles>

Wogonin

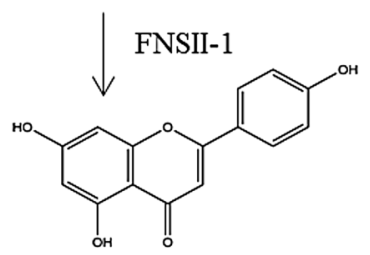

Apigenin

Fig. 2 Main synthetic routes of flavonoids of SB

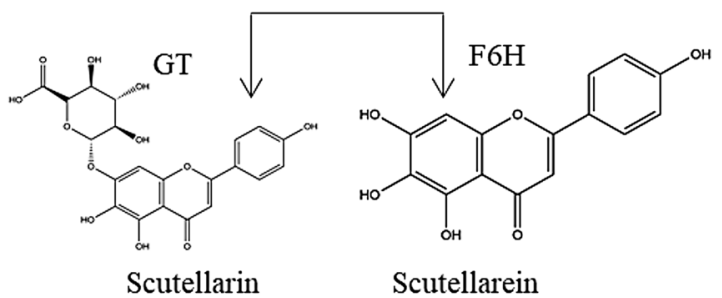


relaxed blood vessels via regulating intracellular $\mathrm{Ca}^{2+}$ in vascular smooth muscle and activating ATP-sensitive potassium channel channels [80].

\section{Anti-diabetics}

SB has great therapeutic potential for T2DM, and its flavonoids are the main components that play a role [81]. $\alpha$-glucosidase inhibitors are currently widely used in the treatment of T2DM. SB contains the above effective ingredients, including 5,7,3,2',6'-pentahydroxyflavanone, baicalin, viscidulin III, $2^{\prime}, 3,5,6^{\prime}, 7$-pentahydroxyflavanone, etc. $[82,83]$. They mainly affected the peroxisome proliferator activated receptor signaling pathway in the development of T2DM. PPAR $\gamma$, PGH2, ACAC $\beta$ and NF-kB subunit 1 are key targets. Other studies had shown that SBE can play an anti diabetes effect by regulating the composition and structure of intestinal microbial. After oral administration of SBE, the composition of intestinal flora, fecal metabolites and SCFAs content changed in T2DM rats [84].

\section{Neuroprotective effect}

Wogonin has a neuroprotective effect on the rat brain after $\gamma$-irradiation [85]. It could restore the mRNA level of cells and the expression of Nrf2, HO-1 and NF- $\mathrm{kB}$. And the lesions of brain tissue structure, such as focal glial degeneration and aggregated astrocytes, also could be treated. SBE can effectively reduce the spinal cord neurons/glial cells and microglial cells peroxide damage and LPS stimulation of injured spinal cord neurons [86]. In LPS-stimulated BV-2 mouse microglia, apigetrin can significantly decrease the production of TNF- $\alpha$, IL-6, PGE2 and NO and inhibit the expression of COX2, iNOS and mRNA [87]. Apgetrin, wogonin and baicalein could inhibit neuronal cell death [88, 89]. SBE could inhibit excitotoxicity induced by lactate dehydrogenase and glutamate, and it also had a stronger inhibitory effect and selectivity on NMDA receptor-mediated toxicity [90]. It suggested that SBE had NMDA receptor-mediated neuroprotective effect in excitotoxic neurons. Flavonoids in stem and leaf of SB could dramatically increase cell survival rate, the activities of SOD, glutathione peroxidase, and $\mathrm{Na}^{+}-\mathrm{K}^{+}$-ATPase, inhibit cell apoptosis and excessive production of MDA in primary cortical neurons exposed to potassium cyanide [91]. In summary, the polyhydroxy structure of flavonoids can protect the brain from hypoxia caused by potassium cyanide or cerebral ischemia and inhibit neuronal apoptosis possibly through neuroinflammation, oxidative stress and nerve injury mutual regulation. And it may have potential preventive effects on neurodegenerative diseases.

\section{Anticancer}

Baicalein showed anti-bladder tumor activity [92]. It could reduce the expression of cyclin B1 and D1 by inhibiting protein synthesis and activation via proteasome degradation and inhibit the expression and activity of MMP-2 and MMP-9 mRNA. SBE can inhibit the proliferation of MCF-7 by inhibiting mitochondrial membrane potential, down-regulating Bcl-2/Bax [93]. SBE could downregulate the expression of caspase family members (e.g. PARP), inhibit proliferation and reduce the mitochondrial membrane potential of AGS cells to induce apoptosis [94]. And it would not show obvious toxicity to normal cells. Cisplatin is an important drug for the treatment of lung cancer, but there are serious side effects (such as severe cachexia and acute kidney injury). In combination with SBE, anticancer effect was enhanced and improved the side effects caused by cisplatin therapy in vivo [95]. Baicalin which is the main component of the SBE possess antitumor activity against all leukemic cell lines especially those with MLL and PBX1 gene rearrangements. Baicalin inhibited cell proliferation, arrested the cell cycle at the G0/G1 phase, and induced cell death through caspase 3/7 activation [96]. Oroxylin A could down-regulate the expression of SHCBP1 (it is an oncogene involved in the development of various cancers) to inhibit the carcinogen-induced malignant transformation of JB6 P + skin epidermal cells and decrease the number of tumor cells [97].

\section{Hepatoprotective effect}

Liver cancer is one of the common cancers, and studies had shown that SB and its active ingredients have great potential effect in the treatment of liver cancer [98]. Baicalein could inhibit the proliferation of HepG2 cells and reduce metastasis by regulating the activity of MMP-2 [99]. In addition, it also could down-regulate the expression of mRNA and protein of CD24 (a key protein in cancer cell proliferation) to induce apoptosis of liver cancer cells [100]. SBE could down-regulate the expression of endoplasmic reticulum stress marker GRP78, and had obvious hepatoprotective effect on acute alcohol-induced liver injury [101]. It could regulate the levels of AST, ALT and TG in the serum, and the levels of GSH and MDA in liver tissues. For non-alcoholic fatty liver, Baicalin downregulated the NLRP3-GSDMD pathway to inhibit liver cell death, but induced cytotoxicity with a doses of $32 \mu \mathrm{M}$ [102].

\section{Immunization effect}

SBE increased the viability of far eastern catfish (Silurus asotus, infected with Vibrio anguillarum or Streptococcus iniae) by regulating growth and serum hormone 
levels [103]. SBWE restored the level of Th2-type IgG1 and Th1-type IgG2a of ligation-induced periodontitis mice through immune response [104]. Haemophilus parasuis can cause a chronic disease related to inflammatory immune response. Baicalin could inhibit the production of IL-6, IL-8, IL-10 and TNF- $\alpha$ and the phosphorylation of ERK, JNK and p38. Thereby activating the immune response induced by Th- 1 to promoting the elimination of bacteria [105]. To sum up, SB play roles of anti-inflammatory, antibacterial and anti-viral via immune response.

\section{Other pharmacological effects}

Anti-aging SBE improved aging symptoms induced by D-galactose, including the learning and memory function, the oxidative damage and histological abnormalities of the hippocampal neurons [106]. It could regulate the disorder of the metabolism of amino acid, glucose and choline. SB flower extract improved the spatial learning and memory ability, regulated the levels of D-glutamine, glutamic acid, MDA, SOD and AGEs [107]. Therefore, SB flower exerted anti-aging effect through regulating glutamate-glutamic acid metabolism pathway. In addition, 5,7,2'-Trihydroxyflavone and scutevulin inhibited senescence-associated secretory phenotype caused by bleomycin with decreasing the expression of IкB $\zeta$ and C/EBP $\beta$ protein [108]. They didn't affect either BrdU uptake or the expression of senescence markers.

Anti-Osteoporosis Tectochrysin could significantly improve the loss of bone trabeculae, reduce bone in serum and decrease CTX-1, TRACP-5b and IL-6 levels to relieve the symptoms of Osteoporosis [109]. SB ether extract (Baicalein and wogonin) could promote osteogenic transformation, further bone regeneration, stromal calcification and calcified nodule formation [110]. And their activity is comparable to SIM $(0.1 \mathrm{mg} / \mathrm{L})$. Baicalin could affect bone metabolism by promoting osteoblast differentiation, inhibiting osteoclast formation, and increasing osteoclast apoptosis [111, 112].

Anti-prostatic hyperplasia SBE improved significantly prostate growth, and increase serum testosterone and $5 \alpha$-reductase levels in prostatic hyperplasia rats by inhibiting the expression of AR and proliferating cell nuclear antigen, restoring the balance of $\mathrm{Bcl}-2 / \mathrm{Bax}$ [113]. These findings enhanced the feasibility of plant extracts to replace commercial $5 \alpha$-reductase inhibitors (such as finasteride).

Anti-Alzheimer MAO-A and MAO-B are considered to treat depression and anxiety drug targets for neuropsychiatric diseases such as Alzheimer and Parkinson [114]. Wogonin and baicalein were observed as effective and selective MAO-A inhibitor [115]. They might be useful lead compound for the development of MAO inhibitors for the treatment of depression, such as Parkinson and Alzheimer. Furthermore, baicalin could promote the differentiation of neurons, which transformation into mature neurons and their survival via the Akt/FOXG1 pathway to exert antidepressant effects [116].

Anti-melanin O-methylated flavones (baicalin, wogonoside, baicalein, wogonin, and oroxylin A) have a dualfunction effect on melanocytes, which are the inhibition of melanin production and intracellular melanosome transport [117]. These flavonoids had structure-specific a-ring and aglycon O-methyl. It indicated that the function of the active ingredient is related to its structure, and the structure-activity relationship is reflected.

Anti-pruritic Baicalin, baicalein and oroxylin A could improved histamine-induced scratching behavior through reducing vascular permeability and contraction [118].

\section{Pharmacokinetics}

Decoction is the main form of TCM used in clinic. Baicalein and wogonin were the major active components in Huangqin decoction [119]. After entering the body, their would be metabolized into glucuronidase and sulfatase forms. After oral administration, the $\mathrm{AUC}_{0-\mathrm{t}}$ of glucuronides/sulfates of wogonin and baicalein reached peak at $10 \mathrm{~min}$. And in serum the content of baicalein and wogonin were ranged from 0.3 to 20 and 0.2 to $10 \mu \mathrm{g} / \mathrm{mL}$, the $C_{\max }$ and $\mathrm{AUC}_{0-\mathrm{t}}$ of baicalein's glucuronides/sulfates at each time point were 3.3 times that of wogonin. The second peak of $\mathrm{AUC}_{0-\mathrm{t}}$ appeared, indicating the occurrence of enterohepatic circulation. The tissues distribution of free baicalein and wogonin were mainly lung and liver respectively. Glucuronides/sulfates of baicalein and wogonin mainly existed in live and kidney respectively. While baicalein and wogonin were mainly present in tissue, their glucuronides/sulfates mainly existed in serum. It suggested that glucuronides/sulfates were involved in circulation. The above components were not detected in brain, suggesting that the above-mentioned components taken orally cannot enter the central nervous system.

SBWE contains mainly 8 flavonoids, baicalin, wogonoside, oroxyloside, norwogonoside, baicalein, wogonin, oroxylin A, norwogonin. After incubation with intestinal bacteria, the content of the latter four components increased significantly, norwogonin had the highest content and showed the highest activity of hemolysis inhibition on sheep and rabbit erythrocytes and anti bacteria while other components didn't work [120]. Another study showed that under pathological conditions intestinal bacteria had stronger glucuronidase activity and a higher 
efficiency in converting SBE to flavonoid aglycones [121]. It indicated that SB can exert pharmacological effects depending on the metabolism of intestinal bacteria to produce effective metabolites.

OG and OS were two metabolites of [122] oroxylin A. OA, OG and OS were quickly and widely distributed in tissues, especially postoperative tissues. OA is more widely distributed in tissues than its metabolites, mainly in liver and kidney. But OA was quickly eliminated in the body and the relative bioavailability was less than $2 \%$. The $\mathrm{AUC}_{0-\mathrm{t}}$ values of them were proportional to dose. After oral administration, OA was mainly excreted from feces, OG was mainly excreted from bile and urine, and OS was almost not excreted.

Clinical data indicated that $100-2800 \mathrm{mg}$ of baicalein in a single oral dose for healthy volunteers is well tolerated, and showed non-toxicity in liver or kidney [123]. And clinical trials had also verified the safety and tolerability it of products currently sold on the market. In summary, most of the ingredients of SB must be metabolized into active compounds by the intestinal flora, such as baicalein and wogonin. In addition, it also shows that some effective ingredients of SB have low oral absorption and availability, short biological half-life, etc. Therefore, how to improve the bioavailability of SB will be the main focus for the follow-up researchers.

\section{Toxicity}

At present, it is generally believed that Chinese herbal medicines have serious side effects, including interstitial pneumonia and liver dysfunction [124]. High dose of wogonin $(40 \mathrm{mg} / \mathrm{kg}$, intravenous injection) significantly increased weight of pregnant mice and structural chromosomal aberrations to affect fetus development [125]. Baicalin inhibited the proliferation of targeted stem cells D3 and 3T3 cells, to exert weak decomposition toxicity [126]. Shuanghuanglian injection had a sensitizing effect, and the allergen component was baicalin [127]. It could activate mast cells and increase the levels of IgE and IgG antibody to cause allergic reaction [128]. It indicated that baicalin could produce specific antibodies IgG and IgE in serum, and thus producing allergic reaction. Studies have also shown that baicalin can induce IgE-mediated pseudo-allergy via Mrgprb2 [129]. Baicalin activated TGF- $\beta$ /Smad signaling pathway to increase kidney collagen synthesis and fibrosis-related protein expression to cause kidney damage and renal fibrosis [130].

SB has more applications in modern skin care industry due to its antioxidant, anti-inflammatory and melanin synthesis inhibitory effects. But the introduction of botanical preparations into cosmetics is an increasing cause of contact dermatitis in patients. There were studies reported that sunscreen containing SB could cause facial inflammation to somebody [131-133]. Therefore, whether SB can be widely used in cosmetics or skin care products still needs further study.

\section{Probably potential therapeutic effect and mechanism of COVID-19}

COVID-19 is a worldwide and severe epidemic at present, caused by SARS-CoV-2 [134]. A research team pointed out that the SARS-CoV-2 virus is similar to the SARS coronavirus [135]. It is suggested that the therapeutic target of SARS can be used as a reference for treatment strategy. Researched showed that angiotensin converting enzyme 2 (ACE2) and coronavirus 3CL Mpro on host epithelial cells affected by its S-protein are considered to be the core targets for inhibiting coronavirus proliferation [136, 137]. Simultaneously, cytokine storm induced by virus is the main cause of complications, such as inflammation, septic shock and multiple organ failure [138].

Baicalin had been confirmed to inhibit SARS-CoV in vitro [139], and scutellarin could interact with ACE2 [140]. At present, the drug research on the treatment for COVID-19 is mainly based on network pharmacology and molecular docking [141]. Baicalein and oroxylin A have a certain binding activity with ACE2 and 2019-nCoV-M $\mathrm{M}^{\mathrm{pro}}$, indicating that they may directly act on the virus and host cells, thus preventing virus proliferation, preventing the body's immunity and blocking virus attack [142-144]. Naringenin and beta-sitosterol can regulate the expression of key genes (CCL2, IL-1 $\beta$ and IL-6) in the treatment of COVID-19, and produce anti-inflammatory and immune enhancing effects through IL-17, TNF, AGE-RAGE signaling pathways and cytokine-cytokine recepter interaction pathway [141]. It is speculated that the therapeutic effects of compounds of SB on COVID-19 mainly focus on anti-inflammatory, inhibiting pro-inflammatory cytokine production and cut of cytokine storm, regulating immune response. Mechanisms of SB in treating COVID-19 shown in Fig. 3. At present, the treatment for COVID-19 researches mainly focus on TCM prescriptions. In addition to the above mentioned, Lianhua Qingwen can regulate the imbalance of ACE-Ang-II and ACE2-Ang-(1-7), which can lead to overwhelming pro-inflammatory cytokines with cytokine storm. And regulating immune-related signal pathway (MAPK, NF-kB, PI3K-AKT, ect) to protect organ damage [145].

To sum up, TCM exhibit functions on COVID-19 via "multi-component, multi-target and multi-pathway". Some countries authorized chloroquine and hydroxychloroquine for the treatment of COVID-19 [146]. But they have adverse reactions, such as diarrhea and nausea, so it is particularly important to seek treatment 


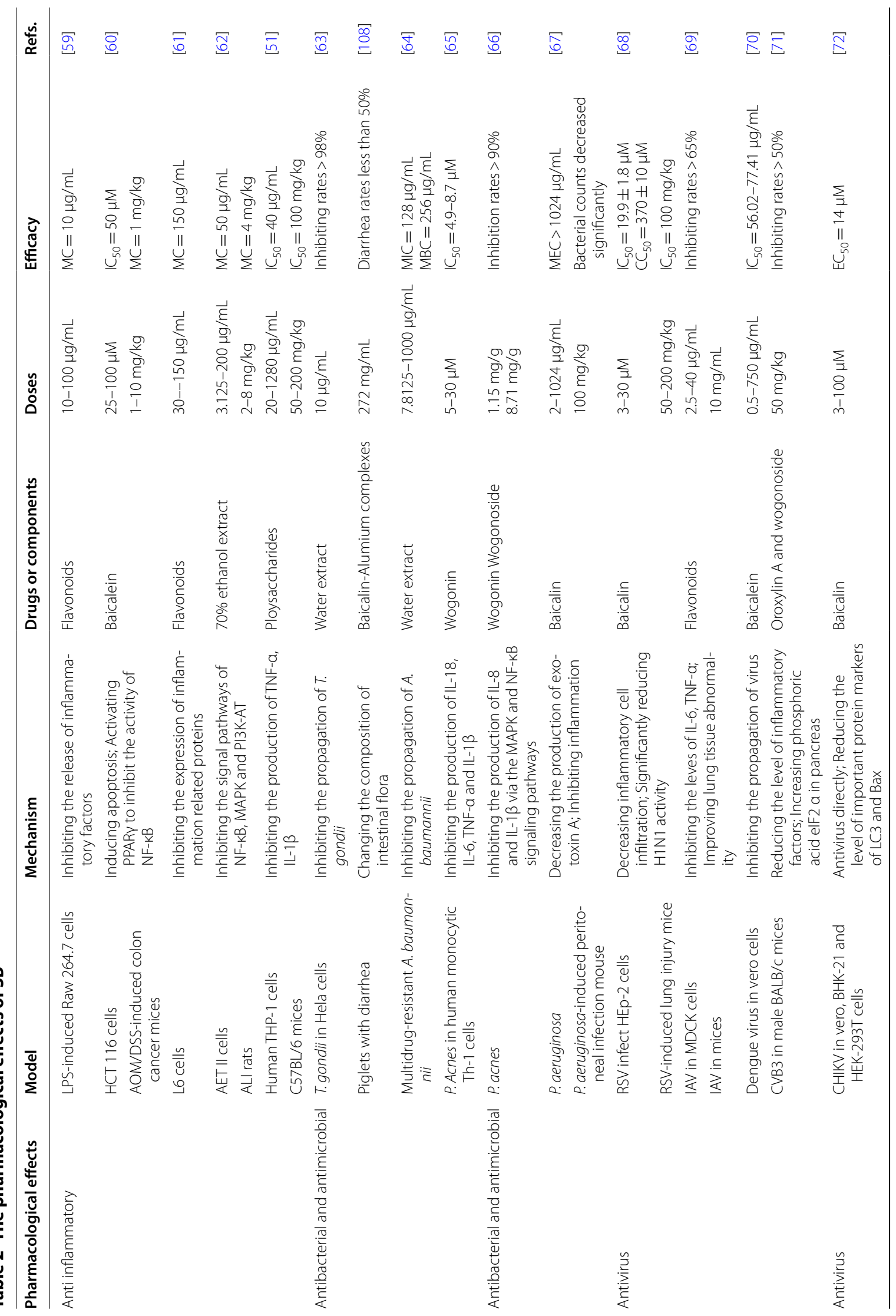




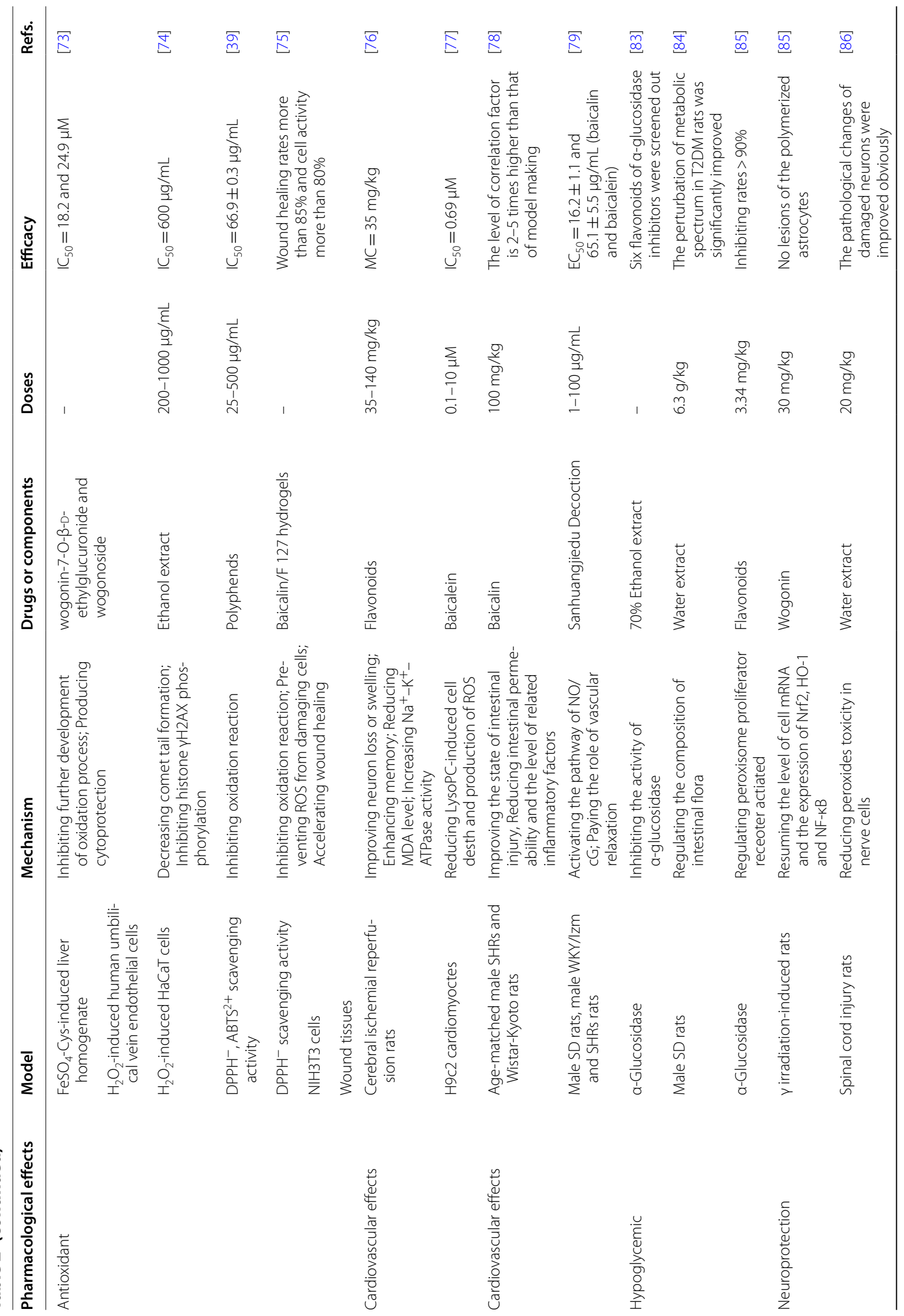




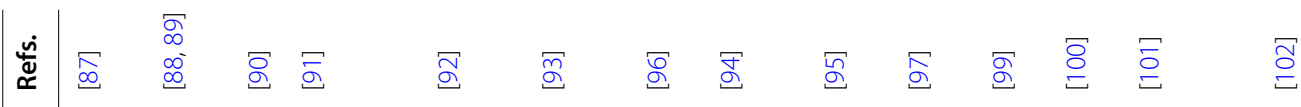

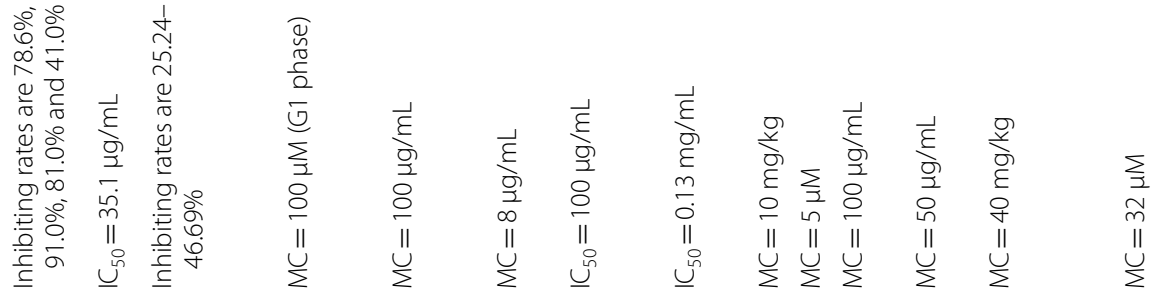

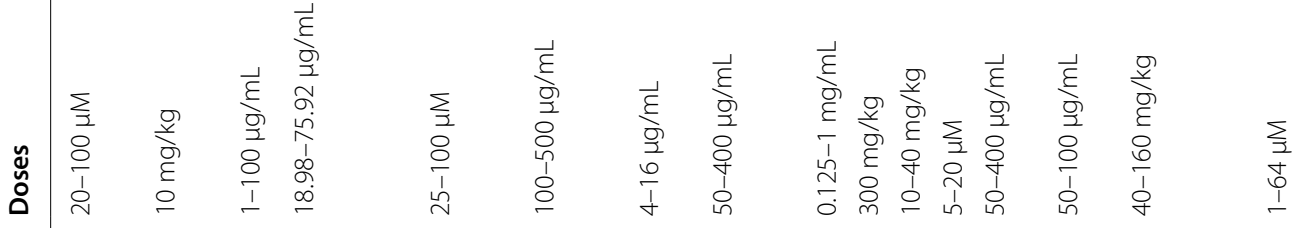
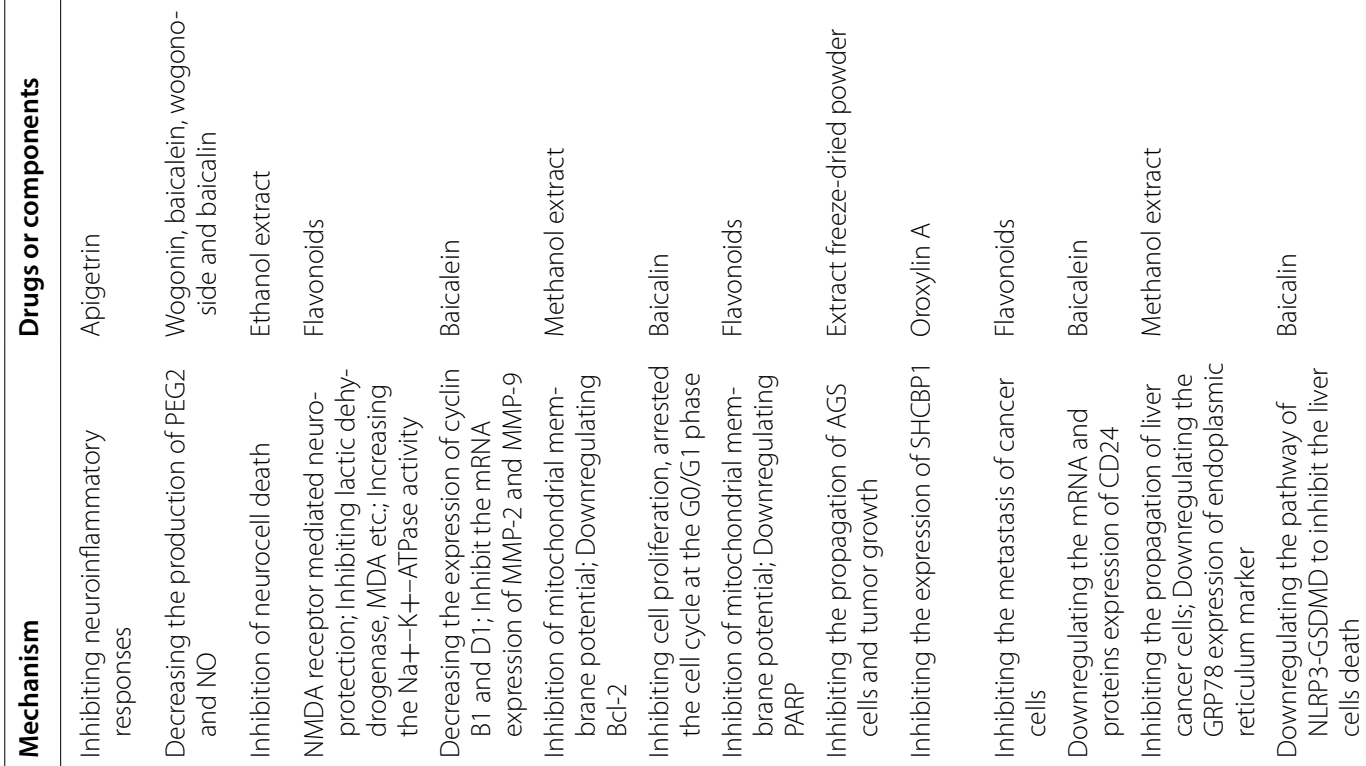

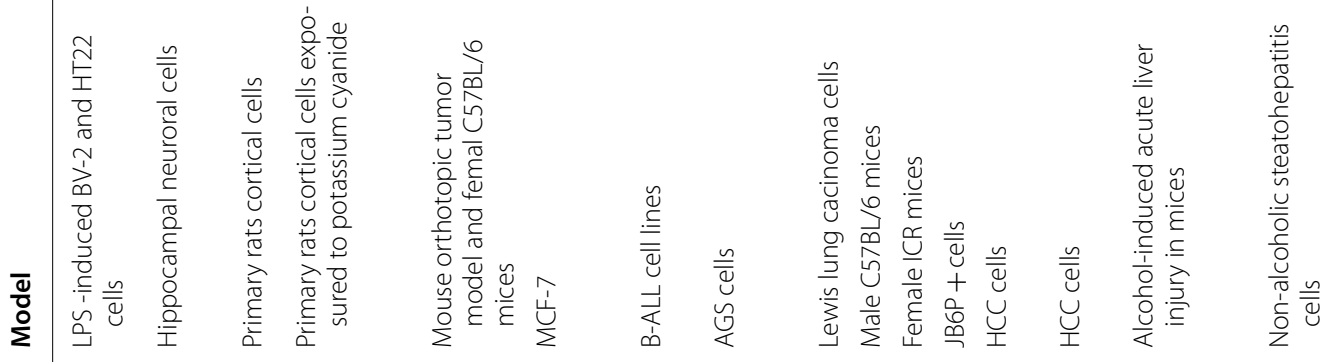
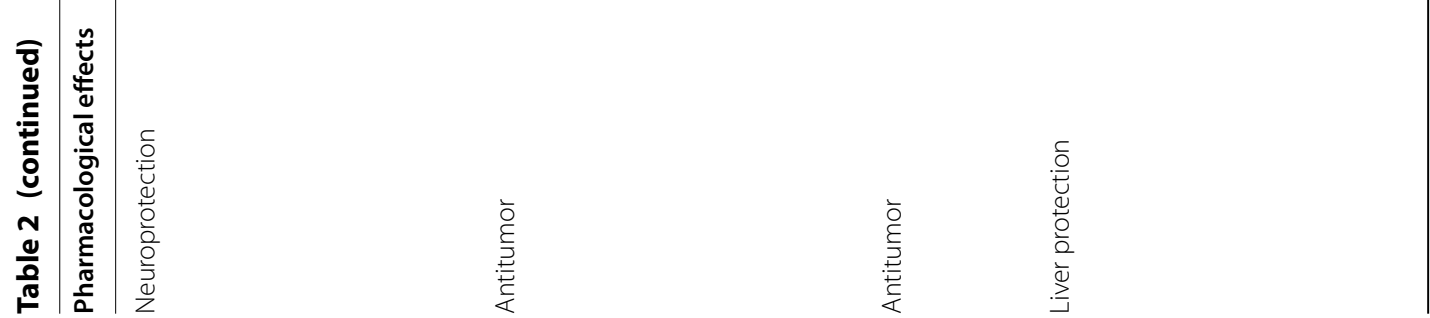


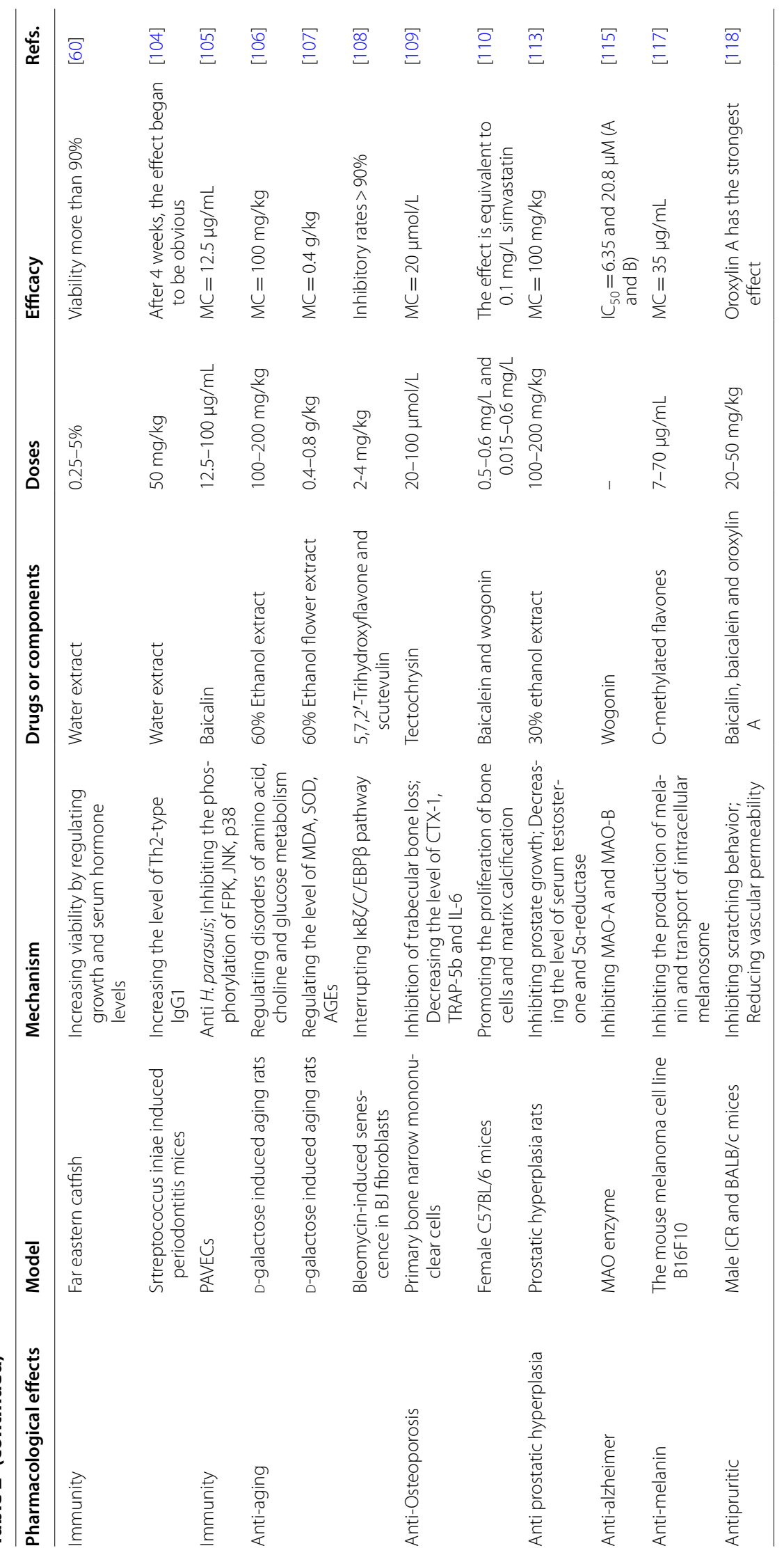




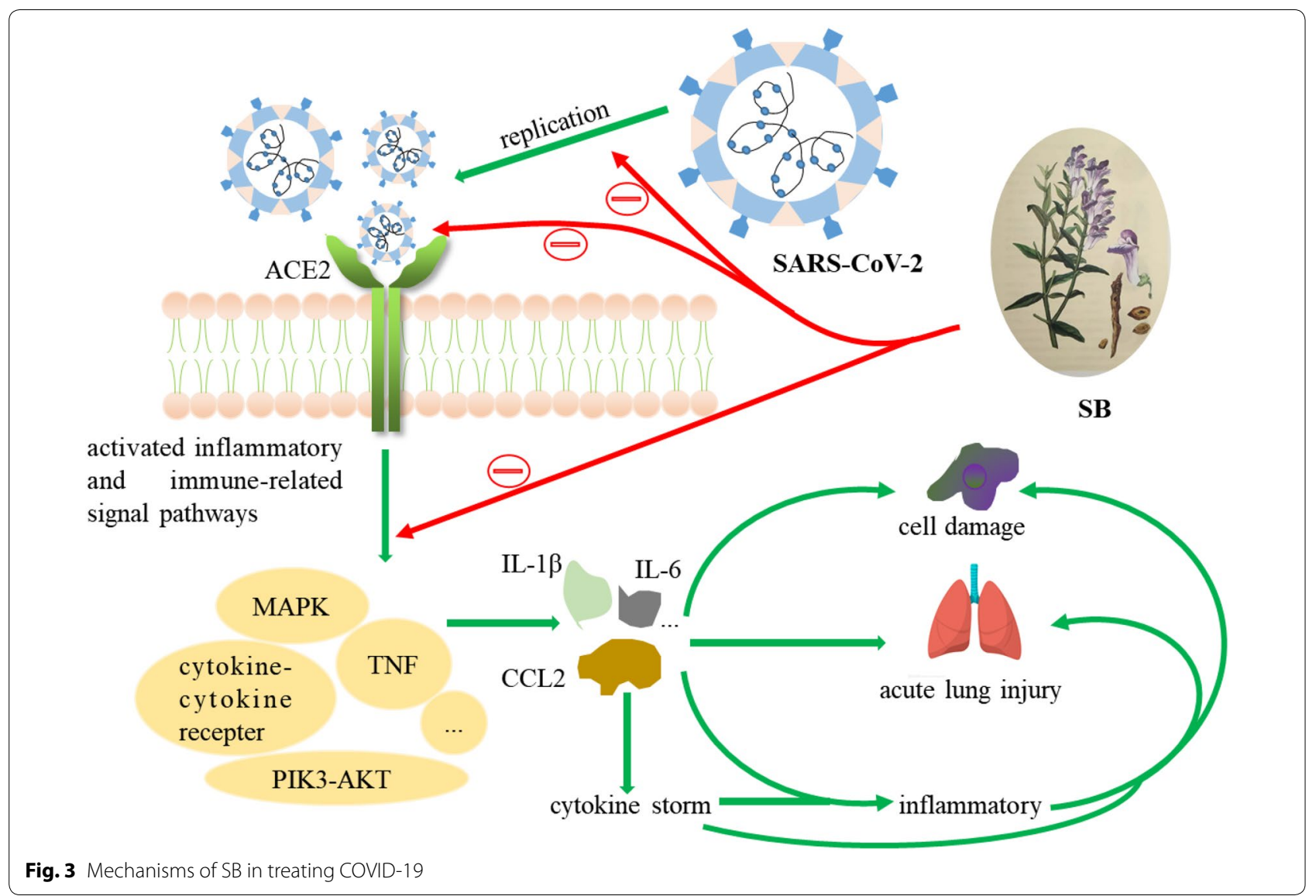

from TCM. In China, there are three formula authorized to treat COVID-19 [147, 148], including Jinhua Qinggan granules, Lianhua Qingwen granules, and Xuebijing injection. At present, the development of accurate and effective therapeutic drugs and vaccines for COVID19 are the research focus of various countries [149]. Although TCM has many advantages mentioned above, its exact effects still needs to be verified by clinical trials.

\section{Conclusion and future perspectives}

$\mathrm{SB}$ is a common TCM with a wide range of clinical effects, and usually used to treat cold, cough, dysentery, lung heat, jaundice and other diseases. According to botanical research, SB in ChP. (1st, 2020) is authentic. In addition to genuine factors, harvest time and processing technology also affect the efficacy of herbs [150]. Despite the commercial interest and increasing demand for SB, improvements through breeding have been very limited. The absence of genome information has limited the understanding of how its flavonoid bioactivities are made and have limited any improvement in productivity through genetic selection. Understanding the genes responsible for biosynthesis of the various flavonoids made in $\mathrm{S}$. baicalensis and their regulation will lay a foundation for molecular breeding for improved, sustainable production.

SB contains a variety of flavonoids, which are the material basis for its strong biological activity, such as baicalin, baicalein, oroxylin $\mathrm{A}$, wogonin, norwogonin and so on. But beyond that, SB also has diterpenes, polyphenols, amino acids, volatile oils, sterols, benzoic acids, etc. Therefore, it have many pharmacological functions such as antibacterial, antiviral, anti-inflammatory, anticancer, liver-protecting and neuroprotective effects. However, the effective components of SB showed low bioavailability and rapid metabolism in vivo. It's suggested that for SB oral preparation, it is necessary to study more suitable technologies to improve its bioavailability in vivo in order to achieve better curative effect. Baicalin is the allergen in $\mathrm{SB}$, which can cause the allergic reaction mediated by IgG and IgE. Therefore, the injection and cream containing baicalin should be strictly controlled to ensure its safety. $\mathrm{SB}$ also has good application prospects in non-medical fields, such as agriculture, industry and beauty industry. Because SB can inhibit the production and transportation of melanin, it is added more in whitening skin care products. The introduction of plant preparations into cosmetics is a relatively risky measure, which may cause 
adverse reactions to users, so its production needs to be strictly controlled.

Baicalin and baicalein had been proved to have inhibitory effect on SARS-CoV in vitro, and scutellarin could also bind with ACE2 receptor to prevent virus invasion. At the same time, they alleviated the complications caused by the virus, through anti-inflammatory, improve immune response and other functions. It is worth noting that the virus will affect the body through multiple pathways and cause many complications. Therefore, compared with the single component, the TCM prescriptions are still the main treatment for COVID-19, and the therapeutic characteristics of "multi-component, multi-target and multi-pathway" of TCM are brought into full play. Due to the lack of understanding of the pathogenesis of COVID-19, symptomatic treatment and alleviation of complications are the main treatment strategies before the development of effective drugs and vaccines. In conclusion, the current researches of SB are summarized, so that readers have a comprehensive understanding of the research extent of $\mathrm{SB}$, and provide ideas for the follow-up study of SB, especially in how to improve the bioavailability of SB oral preparations in vivo.

\begin{abstract}
Abbreviations
PAL: Phenylalanine ammonia lyase; $C 4 \mathrm{H}$ : Cinnamate 4-hydroxylase; CoA: Coenzyme A; CLL: 4-Coumarate CoA ligase; CHS: Chalcone synthase; CHI: Chalcone isomerase; FNSII: Flavone synthase II; MT: Methyltransferases; GT: Glycosyltransferases; FH: Flavone hydroxylase; OMT: O-methyltransferases; DSS: Dextran sodium sulfate; LPS: Lipopolysaccharide; iNOS: Inducible nitric oxide synthase; COX: Cyclooxygenase; IL: Interleukin; TNF-a: Tumor necrosis factoralpha; AOM: Azoxymethane; PPARy: Peroxisome proliferator-activated receptor gamma; NF-kB: Nuclear factor-kappa B; AET II: Alveolar epithelial type II; SBE: SB ethanol extracts; SBWE: SB water extract; MAPK: Mitogen activated protein kinase; MIC: Minimum inhibitory concentration; MBC: Minimum bactericidal concentration; $M C$ : Minimal concentration; $\mathrm{ED}_{50}$ : Median effective dose; $\mathrm{IC}_{50}$ : Half maximal inhibitory concentration; HA: Hemagglutinin; NA: Neuraminidase; H2AFX: H2A histone family member X; ROS: Reactive oxygen species; Nrf2/HO-1: Nuclear factor-erythroid 2-related factor 2/Heme oxygenase 1; SOD: Superoxide dismutase; MDA: Malondialdehyde; LysoPC: Lysophosphatidylcholine; BCl-2: B-cell lymphoma 2; Bax: BCl-2-associated X protein; SCFAs: Short-chain fatty acids; NO/cGMP: Nitric oxide/cyclic Guanosine monophosphate; BKCa: Large conductance calcium-activated potassium channels; DAG/ PKC/CPI-17: Diacylglycerol/Protein kinase C/Cytosolic protein of 17 kDa; T2DM: Type 2 diabetes; PGH2: Prostaglandin G/H synthase 2; ACAC $\beta$ : Acetyl-CoA carboxylase beta; PGE2: Prostaglandin E2; NMDA: N-methyl D-aspartate; MMP: Matrix metalloproteinase; PARP: Poly (ADP-ribose) polymerase; FOXM1: Forkhead Box M1 activity; AST: Aspartate transaminase; ALT: Alanine transferase; TG: Triglyceride; GSH: Glutathione; GRP78: 78-kDa glucose-regulated protein; NLRP3-GSDMD: NLR pyrin domain containing 3-gasdermin D; PAVECs: Porcine aortic vascular endothelial cells; JNK: c-JUN N-terminal kinase; ERK: Extracellular signal-regulated kinase; AGEs: Advanced glycation end products; SIM: Simvastatin; CTX-1: TRACP-5b: Bone turnover markers; MAO: Monoamine oxidase; ALI: Acute lung injury; OG: Oroxylin A 7-O-glucuronic acid; OS: Oroxylin A sodium sulfonate; D3: Embryonic stem cell; 3T3: Embryonic fibroblast; TGF- $\beta$ : Transforming growth factor- $\beta$; Smad: The main signal transducers for receptors of the TGF- $\beta$; SARS-CoV-2: Severe acute respiratory syndrome coronavirus 2; BrdU: 5-Bromo-2'-deoxy-uridine.
\end{abstract}

Acknowledgements

Not applicable.

\section{Authors' contributions}

J-WS wrote the manuscript. J-WS and J-YL systemically revised the manuscript for important content. LX and L-LZ completed the Figures and Tables. Q-XX, $\mathrm{H}-\mathrm{JC}$ and MD collected literature and checked data and X-FL proposed the conception and designed the structure of the manuscript. All authors read and approved the final manuscript.

\section{Funding}

The authors would like to acknowledge the financial support from the Science and Technology Department of Sichuan Province (No. 2019YFS0113) and State Administration of Traditional Chinese Medicine (No. 2018ZYO3O02).

\section{Availability of data and materials}

Not applicable.

\section{Ethics approval and consent to participate}

Not applicable.

\section{Consent for publication}

The manuscript is approved by all authors for publication.

\section{Competing interests}

The authors declare that they have no competing interests.

Received: 10 July 2020 Accepted: 18 September 2020

Published online: 25 September 2020

\section{References}

1. Commission CP. Pharmacopoeia of the People's Republic of China Part. I. Beijing: People's Medical Publishing House; 2020.

2. Shang X, He X, He X, Li M, Zhang R, Fan P. The genus Scutellaria an ethnopharmacological and phytochemical review. J Ethnopharmacol. 2010;128:279-313.

3. Liu ZB, Sun CP, Xu JX, Morisseau C, Hammock BD, Qiu F. Phytochemical constituents from Scutellaria baicalensis in soluble epoxide hydrolase inhibition: kinetics and interaction mechanism merged with simulations. Int J Biol Macromol. 2019;133:1187-93.

4. Dinda B, Dinda S, Dassharma S, Banik R, Chakraborty A, Dinda M. Therapeutic potentials of baicalin and its aglycone, baicalein against inflammatory disorders. Eur J Med Chem. 2017;131:68-80.

5. Yan B, Xu W, Su S, Zhu S, Zhu Z, Zeng H, Zhao M, Qian D, Duan JA, Duan J. Comparative analysis of 15 chemical constituents in Scutellaria baicalensis stem-leaf from different regions in China by ultra-high performance liquid chromatography with triple quadrupole tandem mass spectrometry. J Sep Sci. 2017;40:3570-81.

6. Zhao Q, Yang J, Cui MY, Liu J, Fang YM, Yan MX, Qiu WQ, Shang HW, Xu ZC, Yidiresi R, Weng JK, Pluskal T, Vigouroux M, Steuernagel B, Wei YK, Yang L, Hu YH, Chen XY, Martin C. The reference genome sequence of Scutellaria baicalensis provides insights into the evolution of Wogonin biosynthesis. Mol Plant. 2019;12:935-50.

7. Shi L, Hao Z, Zhang S, Wei M, Lu B, Wang Z, Ji L. Baicalein and baicalin alleviate acetaminophen-induced liver injury by activating Nrf2 antioxidative pathway: the involvement of ERK1/2 and PKC. Biochem Pharmacol. 2018;150:9-23.

8. Wang QQ, Xu HO, Zhao XF. Baicalin inhibits human cervical cancer cells by suppressing protein kinase C/signal transducer and activator of transcription (PKC/STAT3) signaling pathway. Med Sci Monit. 2018;24:1955-61

9. Ma QH, Ren MY, Luo JB. San Wu Huangqin decoction regulates inflammation and immune dysfunction induced by influenza virus by regulating the NF-KB signaling pathway in $\mathrm{H} 1 \mathrm{~N} 1$-infected mice. J Ethnopharmacol. 2021;264:112800.

10. Xiao SW, Zhang ZM, Chen MJ, Zou JF, Jiang S, Qian DW, Duan JN. Xiexin Tang ameliorates dyslipidemia in high-fat diet-induced obese rats via elevating gut microbiota-derived short chain fatty acids production and adjusting energy metabolism. J Ethnopharmacol. 2019;241:112032.

11. Zhang JF. 4 cases of skin diseases treated by adding or subtracting the antiseptic Dan of manna. Hunan J Tradit Chin Med. 2016;36:89-90. 
12. Jia Q, Shen D, Tang SH, Li DF, Zhang YH. Analysis of the law of the use of proprietary medicine containing Scutellaria baicalensis root. Chin J Chin Materia Med. 2014;39:634-8.

13. Hong HD, Liu CX, Hong Y, Huang HT, Li DT, Pan Y, Chen S, Chen CR. Mining and analysis of the formulation rules of Chinese patent medicine for cold based on 2015 Edition of Chinese Pharmacopeia (Part I). China Pharm. 2019;30:1812-6.

14. Commission CP. Pharmacopoeia of the People's Republic of China Part IV. Beijing: People's Medical Publishing House; 2020.

15. Song J, Ke R, Zhang M, Fei G, Ma X, Qiu J. Interface interaction and compatibility molecular dynamics verification of Scutellaria baicalensis Georgi extracts/PBS dyeing and antibacterial composites. Mater Res Express. 2019;6:075403.

16. Zhang S, Qu Z, Hsueh CC, Chang CT, Chen BY. Deciphering electronshuttling characteristics of Scutellaria baicalensis Georgi and ingredients for bioelectricity generation in microbial fuel cells. J Taiwan Inst Chem Eng. 2019;96:361-73.

17. Liao XD, Wen Q, Zhang LY, Lu L, Zhang LY, Luo XG. Effect of dietary supplementation with flavonoid from Scutellaria baicalensis Georgi on growth performance, meat quality and antioxidative ability of broilers. J Integr Agric. 2018;17:1165-70.

18. Zhou Y, Yang ZY, Tang RC. Bioactive and UV protective silk materials containing baicalin - the multifunctional plant extract from Scutellaria baicalensis Georgi. Mater Sci Eng C. 2016:67:336-44.

19. Kim HM, Lim YY, Cho SM, Kim MY, Son IP, Suk JM, Park JO, Park JH, Cho JW. The evaluation of skin safety and skin cell toxicity for Scutellaria baicalensis Georgi extract according to extraction conditions. Korean J Dermatol. 2012;50:959-68.

20. Xu N, Meng FY, Zhou GF, Li YF, Wang B, Lu H. Assessing the suitable cultivation areas for Scutellaria baicalensis in China using the Maxent model and multiple linear regression. Biochem Syst Ecol. 2020;90:104052

21. Cao X, You G, Li H, Li D, Wang M, Ren X. Comparative investigation for rotten xylem (kuqin) and strip types (tiaoqin) of Scutellaria baicalensis Georgi based on fingerprinting and chemical pattern recognition. Molecules. 2019:24:2431.

22. Chen $B H$, Chen BX. Research progress of radix scutellariae. World Latest Med Inf. 2019:19:132-4.

23. Sandanov DV, Rosbakh S. Demographic structure of Scutellaria baicalensis Georgi depending on climatic gradients and local factors. Russ J Ecol. 2019;50:404-7.

24. Zhang T, Cheng L, Yang LL, Lin HM, Yang LM, Han M. Effects of ecological factors and gene expression of key enzymes on synthesis of major medicinal ingredients of Scutellaria baicalensis in autumn. Chin Tradit Herb Drugs. 2019;50:936-44.

25. Liu SL, Chen JB, Zhou Q, Sun SQ. Analysis of the harvest seasons of Scutellaria Baicalensis Georgi by tri-step identification of infrared spectroscopy and principal component analysis. Spectrosc Spectr Anal. 2012;32:2669-73.

26. Cheng L, Han M, Yang LM, Li Y, Sun Z, Zhang T. Changes in the physiological characteristics and baicalin biosynthesis metabolism of Scutellaria baicalensis Georgi under drought stress. Ind Crops Prod. 2018;122:473-82

27. Sun C, Zhang M, Dong H, Liu W, Guo L, Wang X. A spatially-resolved approach to visualize the distribution and biosynthesis of flavones in Scutellaria baicalensis Georgi. J Pharm Biomed Anal. 2020;179:113014.

28. Treutter D. Significance of flavonoids in plant resistance andenhancedment of their biosynthesis. Plant Biol. 2005;7:581-91.

29. Nagashima S, Hirotani M, Yoshikawa T. Purification and characterization of UDP- glucuronate Baicalein 7-O-glucuronosyltransferase from Scutellaria baicalensis Georgi cell suspension cultures. Phytochemistry. 2000:53:533-8.

30. Su H, Song S, Yan X, Fang L, Zeng B, Zhu Y. Endogenous salicylic acid shows different correlation with baicalin and baicalein in the medicinal plant Scutellaria baicalensis Georgi subjected to stress and exogenous salicylic acid. PLOS ONE. 2018;13:1-16.

31. Chai CC, Cao Y, Mao M, Wang JY, Liu N, Li XX, Zhang K, Chen DL, Wei LY. Evaluation of taste changes after wine-frying of Scutellariae Radix based on electronic ongue technology and its application in identification of Scutellariae Radix pieces. Chin J Chin Mater Med. 2020:45:2552-9.
32. Yuan Y, Liu Y, Wu C, Chen S, Wang Z, Yang Z. Water deficit affected flavonoid accumulation by regulating hormone metabolism in Scutellaria baicalensis Georgi roots. PLoS ONE. 2012;7:1-10.

33. Tang WT, Fang MF, Liu X, Yue M. Simultaneous quantitative and qualitative analysis of flavonoids from ultraviolet-B radiation in leaves and roots of Scutellaria baicalensis Georgi Using LC-UV-ESI-Q/TOF/MS. J Anal Methods Chem. 2014;2014.

34. Su H, Yu C, Shang J, Yan X, Liao P, Zhu Y. Effects of endogenous salicylic acid synthesized through PAL and ICS pathway on baicalin and baicalein accumulation in Scutellaria baicalensis Georgi. Acta Physiol Plant. 2016:38:1-9.

35. Anna YS, Alexandra LS, Svetlana AS. Influence of spectral light composition on flavones formation in callus culture of Scutellaria baicalensis Georgi. Pharmacogn Mag. 2020;16:156-60.

36. Zhao T, Tang H, Xie L, Zheng Y, Ma Z, Sun Q. Scutellaria baicalensis Georgi. (Lamiaceae): a review of its traditional uses, botany, phytochemistry, pharmacology and toxicology. J Pharm Pharmacol. 2019:71:1353-69.

37. Ji S, Li R, Wang Q, Miao WJ, Li ZW, Si LL, Qiao X, Yu SW, Zhou DM, Ye M. Anti-H1N1 virus, cytotoxic and Nrf2 activation activities of chemical constituents from Scutellaria baicalensis. J Ethnopharmacol. 2015;176:475-84.

38. Wang ZL, Wang S, Kuang Y, Hu ZM, Qiao X, Ye M. A comprehensive review on phytochemistry, pharmacology, and flavonoid biosynthesis of Scutellaria baicalensis. Pharm Biol. 2018;56:465-84.

39. Seo ON, Kim GS, Kim YH, Park S, Jeong SW, Lee SJ, Jin JS, Shin SC. Determination of polyphenol components of Korean Scutellaria baicalensis Georgi using liquid chromatography-tandem mass spectrometry: contribution to overall antioxidant activity. J. Funct. Foods. 2013;5:1741-50.

40. Zhang BY. Study on Chemical Constituents of the aerial parts of Scutellaria baicalensis Georgi. Qiqihaer University; 2012.

41. Gharari Z, Bagheri K, Danafar H, Sharafi A. Enhanced flavonoid production in hairy root cultures of Scutellaria bornmuelleri by elicitor induced over-expression of MYB7 and FNSIII genes. Plant Physiol Biochem. 2020;148:35-44.

42. Zhao Q, Cui MY, Levsh O, Yang DF, Liu J, Li J, Hill L, Yang L, Hu YH, Weng JK, Chen XY, Martin C. Two CYP82D enzymes function as flavone hydroxylases in the biosynthesis of root-specific 4'-deoxyflavones in Scutellaria baicalensis. Mol Plant. 2018;1 1:135-48.

43. Huang GQ, Liang J, Lin J, Chen XSI, Xin CX, Yang CC. Predictive analysis of quality markers: a case study of Scutellariae Radix formula. Chinese J New Drugs. 2020;29:285-92.

44. Zhang $\mathrm{GM}, \mathrm{Xu} X \mathrm{XY}$, Wang $\mathrm{XH}$. A method for regulating the ratio of baicalin and baicalein in baicalin extract. Shanghai Inst Tradit Chin Med. 2005.

45. Song SH, Wang Z. Analysis of essential oils from different organs of Scutellaria baicalensis. J Chin Med Mater. 2010:33:1265-70.

46. Qi Y, Zhang Q, Zhu H. Huang-Lian Jie-Du decoction: a review on phytochemical, pharmacological and pharmacokinetic investigations. Chin Med. 2019;14:1-22.

47. Yao G, Wang Y, Liu Y, Tao Y, Dai WF, Wang H. Total flavonoids from Scutellaria baicalensis and total iridoid glycosides from Gardenia jasminoides inhibit influenza H1N1 in vitro. Chin Tradit Pat Med. 2014:36:698-702.

48. Olennikov DN, Chirikova NKTL. Lamiaceae carbohydrates. IV. Watersoluble polysaccharides from Scutellaria baicalensis. Chem Nat Compd. 2011:44:556-9.

49. Olennikov DN, Rokhin AV, Tankhaeva LM. Lamiaceae carbohydrates. V. Structure of glucoarabinogalactan from Scutellaria baicalensis. Chem Nat Compd. 2011:44:560-3.

50. Olennikov DN, Stolbikova AV, Rokhin AV, Khobrakova VB. Carbohydrates from Lamiaceae. VIII. a-glucan from Scutellaria baicalensis roots. Chem Nat Compd. 2011:47:190-3.

51. Cui L, Wang W, Luo Y, Ning Q, Xia Z, Chen J. Polysaccharide from Scutellaria baicalensis Georgi ameliorates colitis via suppressing NF-KB signaling and NLRP3 inflammasome activation. Int J Biol Macromol. 2019;132:393-405.

52. Yan BF, Zhu SQ, Su SL, Zhu ZH, Guo S, Zeng HT, Qian DW, Duan JF. Simultaneous determination of amino acids in Scutellaria baicalensis stem-leaf from different habitats by UPLC-TQ-MS. Chin J Pharm Anal. 2018;38:1165-73. 
53. Vergun O, Svydenko L, Grygorieva O, Shymanska O, Rakhmetov D, Brindza J. Antioxidant capacity of plant raw material of Scutellaria baicalensis Georgi. Potravin Slovak J Food Sci. 2019;13:614-21.

54. Tuan PA, Kim YB, Kim JK, Arasu MV, Al-Dhabi NA, Park SU. Molecular characterization of carotenoid biosynthetic genes and carotenoid accumulation in Scutellaria baicalensis Georgi. EXCLI J. 2015;14:146-57.

55. Long $H$, Zhang $H$, Deng $A$, Ma L, Wu L, Li Z. Three new lignan glucosides from the roots of Scutellaria baicalensis. Acta Pharm Sin B. 2016:6:229-33.

56. Pshenichkina YA, Pshenichkin AY. Biogeochemical features of platinum accumulation in Scutellaria baicalensis Georgi (Lamiaceae). Contemp Probl Ecol. 2018;11:221-6.

57. Tuo L, Yan XR, Li FN, Bao YX, Shi HC, Li HY. Brachybacterium endophyticum sp. nov., a novel endophytic actinobacterium isolated from bark of Scutellaria baicalensis Georgi. Int J Syst Evol Microbiol. 2018;68:3563-8.

58. Pu WL, Bai RY, Zhou K, Peng YF, Zhang MY, Hottiger M, Li WH, Gao XM, Sun LK. Baicalein attenuates pancreatic inflammatory injury through regulating MAPK, STAT 3 and NF-KB activation. Int Immunopharmacol. 2019;72:204-10

59. Hong GE, Kim JA, Nagappan A, Yumnam S, Lee HJ, Kim EH. Flavonoids identified from Korean scutellaria baicalensis georgi inhibit inflammatory signaling by suppressing activation of NF-KB and MAPK in raw 264.7 cells. Evid-Based Compl Alt. 2013;2013.

60. Kim DH, Hossain MA, Kang YJ, Jang JY, Lee YJ, Im E. Baicalein, an active component of Scutellaria baicalensis Georgi, induces apoptosis in human colon cancer cells and prevents AOM/DSS-induced colon cancer in mice. Int J Oncol. 2013:43:1652-8.

61. Kim JA, Nagappan A, Park HS, Venkatarame Gowda Saralamma V, Hong GE, Yumnam S. Proteome profiling of lipopolysaccharide induced L6 rat skeletal muscle cells response to flavonoids from Scutellaria baicalensis Georgi. BMC Complem Altern M. 2014;14:1-10.

62. Feng T, Zhou L, Gai S, Zhai Y, Gou N, Wang X. Acacia catechu (L.f.) Willd and Scutellaria baicalensis Georgi extracts suppress LPS-induced pro-inflammatory responses through NF-KB, MAPK, and PI3K-Akt signaling pathways in alveolar epithelial type II cells. Phyther Res. 2019:33:3251-60

63. Yang $X$, Huang $B$, Chen J, Huang S, Zheng H, Lun ZR. In vitro effects of aqueous extracts of Astragalus membranaceus and Scutellaria baicalensis GEORGI on Toxoplasma gondii. Parasitol Res. 2012;110:2221-7.

64. Miyasaki Y, Rabenstein JD, Rhea J, Crouch ML, Mocek UM, Kittell PE. Isolation and characterization of antimicrobial compounds in plant extracts against multidrug-resistant Acinetobacter baumannii. PLoS ONE. 2013;8:2-9.

65. Zhu XJ, Mao Y, Guo MM, Yu HY, Hao LL, Hua Q, Lu Z, Hong MH, An FL. Enhancement of anti-acne effect of Scutellaria baicalensis extract by fermentation with symbiotic fungus Penicillium decumbens. J Biosci Bioeng. 2020.

66. Luo J, Dong B, Wang K, Cai S, Liu T, Cheng X. Baicalin inhibits biofilm formation, attenuates the quorum sensing-controlled virulence and enhances Pseudomonas aeruginosa clearance in a mouse peritoneal implant infection model. PLoS ONE. 2017;12:e0176883.

67. Fu SL, Zhuang F, Guo L, Qiu YS, Xiong JL, Ye C, Liu Y, Wu ZY, Hou YQ. Effect of baicalin-aluminum complexes on fecal microbiome in piglets. Int J Mol Sci [Internet]. 2019;22:2390.

68. Shi H, Ren K, Lv B, Zhang W, Zhao Y, Tan RX. Baicalin from Scutellaria baicalensis blocks respiratory syncytial virus (RSV) infection and reduces inflammatory cell infiltration and lung injury in mice. Sci Rep. 2016:6:1-12.

69. Zhi HJ, Zhu HY, Zhang YY, Lu Y, Li H, Chen DF. In vivo effect of quantified flavonoids-enriched extract of Scutellaria baicalensis root on acute lung injury induced by influenza A virus. Phytomedicine. 2019;57:105-16.

70. Zandi K, Lim TH, Rahim NA, Shu MH, Teoh BT, Sam SS. Extract of Scutellaria baicalensis inhibits dengue virus replication. BMC Complement Altern Med. 2013;13:91.

71. Kwon BE, Song JH, Song HH, Kang JW, Hwang SN, Rhee KJ. Antiviral activity of oroxylin A against coxsackievirus B3 alleviates virus-induced acute pancreatic damage in mice. PLoS ONE. 2016;11:1-13.

72. Oo A, Rausalu K, Merits A, Higgs S, Vanlandingham D, Bakar SA. Deciphering the potential of baicalin as an antiviral agent for Chikungunya virus infection. Antiviral Res. 2018;150:101-11.
73. Wang MH, Li LZ, Sun JB, Wu FH, Liang JY. A new antioxidant flavone glycoside from Scutellaria baicalensis Georgi. Nat Prod Res. 2014;28:1772-6.

74. Yoon JJ, Jeong JW, Choi EO, Kim MJ, Hwang-Bo H, Kim HJ. Protective effects of Scutellaria baicalensis Georgi against hydrogen peroxideinduced DNA damage and apoptosis in $\mathrm{HaCaT}$ human skin keratinocytes. EXCLI J. 2017;16:426-38.

75. Liu G, Bao Z, Wu J. Injectable baicalin/F127 hydrogel with antioxidant activity for enhanced wound healing. Chinese Chem Lett. 2020:31:1817-21.

76. Shang YZ, Zhang H, Cheng JJ, Miao H, Liu YP, Cao K. Flavonoids from Scutellaria baicalensis Georgi are effective to treat cerebral ischemia/ reperfusion. Neural Regen Res. 2013;8:514-22.

77. Chen HM, Hsu JH, Liou SF, Chen TJ, Chen LY, Chiu CC. Baicalein, an active component of Scutellaria baicalensis Georgi, prevents lysophosphatidylcholine-induced cardiac injury by reducing reactive oxygen species production, calcium overload and apoptosis via MAPK pathways. BMC Complem Altern Med. 2014;14:1-10.

78. Wu D, Ding L, Tang X, Wang W, Chen Y, Zhang T. Baicalin protects against hypertension-associated intestinal barrier impairment in part through enhanced microbial production of short-chain fatty acids. Front Pharmacol. 2019;10:1-13.

79. Wu J, Nakashima S, Shigyo M, Yamasaki M, Ikuno S, Morikawa A. Antihypertensive constituents in Sanoshashinto. J Nat Med. 2020;74:421-33.

80. Ding LQ, Jia CL, Zhang Y, Wang WJ, Zhu WL, Chen Y, Zhang T. Baicalin relaxes vascular smooth muscle and lowers blood pressure in spontaneously hypertensive rats. Biomed Pharmacother. 2019;111:325-30.

81. Xu LN, Li Y, Dai Y. Natural products for the treatment of type 2 diabetes mellitus: pharmacology and mechanisms. Pharmacol Res. 2018;130:451.

82. Yang ZC, Huang W, Zhang JS, Xie M, Wang XW. Baicalein improves glucose metabolism in insulin resistant HepG2 cells. Eur J Pharmacol. 2019:854:187-93.

83. Wang L, Tan N, Wang H, Hu J, Diwu W, Wang X. A systematic analysis of natural a-glucosidase inhibitors from flavonoids of Radix scutellariae using ultrafiltration UPLC-TripleTOF-MS/MS and network pharmacology. BMC Complem Med Ther. 2020;20:1-17.

84. Xiao S, Liu C, Chen M, Zou J, Zhang Z, Cui X. Scutellariae radix and coptidis rhizoma ameliorate glycolipid metabolism of type 2 diabetic rats by modulating gut microbiota and its metabolites. Appl Microbiol Biotechnol. 2020;104:303-17.

85. Wang L, Li C, Sreeharsha N, Mishra A, Shrotriya V, Sharma A. Neuroprotective effect of Wogonin on Rat's brain exposed to gamma irradiation. J Photochem Photobiol B Biol. 2020;204:111775.

86. Tu TH, Liou DY, Lin DY, Yang HC, Chen CJ, Huang MC, Huang WC, Tsai MJ. Characterizing the neuroprotective effects of S/B remedy (Scutellaria baicalensis Georgi and Bupleurum scorzonerifolfium Willd) in spinal cord injury. Molecules. 2019;24:1885.

87. Lim HS, Kim OS, Kim BY, Jeong SJ. Apigetrin from Scutellaria baicalensis georgi inhibits neuroinflammation in BV-2 microglia and exerts neuroprotective effect in HT22 hippocampal cells. J Med Food. 2016;19:1032-40.

88. Gaire BP, Kim YO, Jin ZH, Park J, Choi H, Bu Y. Neuroprotective effect of Scutellaria baicalensis flavones against global ischemic model in rats. J Nepal Pharm Assoc. 2015;27:1-8.

89. Gaire B, Song J, Lee S, Kim H. Neuroprotective effect of four flavonoids in the root of Scutellaria baicalensis Georgi. Planta Med. 2012;78:PF71.

90. Yang J, Wu X, Yu H, Liao X, Teng L. NMDA receptor-mediated neuroprotective effect of the Scutellaria baicalensis georgi extract on the excitotoxic neuronal cell death in primary rat cortical cell cultures. Sci World J. 2014;2014.

91. Miao G, Zhao H, Guo K, Cheng J, Zhang S, Zhang X. Mechanisms underlying attenuation of apoptosis of cortical neurons in the hypoxic brain by flavonoids from the stems and leaves of Scutellaria baicalensis Georgi. Neural Regen Res. 2014;9:1592-8.

92. Wu JY, Tsai KW, Li YZ, Chang YS, Lai YC, Laio YH. Anti-bladder-tumor effect of baicalein from Scutellaria baicalensis georgi and its application in vivo. Evid-based Complem Altern M. 2013;2013.

93. Park JR, Lee MC, Moon SC, Kim J, Ha KT, Park EJ. Scutellaria baicalensis Georgi induces caspase-dependent apoptosis via mitogen activated protein kinase activation and the generation of reactive oxygen species signaling pathways in MCF-7 breast cancer cells. Mol Med Rep. 2017:16:2302-8. 
94. Saralamma WG, Lee HJ, Hong GE, Park HS, Yumnam S, Raha S. Korean Scutellaria baicalensis georgi flavonoid extract induces mitochondrially mediated apoptosis in human gastric cancer AGS cells. Oncol Lett. 2017;14:607-14

95. Huang TH, Wu TH, Guo YH, Li TL, Chan YL, Wu CJ. The concurrent treatment of Scutellaria baicalensis Georgi enhances the therapeutic efficacy of cisplatin but also attenuates. Chemotherapy-induced cachexia and acute kidney injury. J Ethnopharmacol. 2019;243:112075.

96. Orzechowska BU, Wróbel G, Turlej E, Jatczak BN, Sochocka M, Chaber R. Antitumor effect of baicalin from the Scutellaria baicalensis radix extract in B-acute lymphoblastic leukemia with different chromosomal rearrangements. Int Immunopharmacol. 2020;79:106114.

97. Huang H, Cai H, Zhang L, Hua Z, Shi J, Wei Y. Oroxylin A inhibits carcinogen-induced skin tumorigenesis through inhibition of inflammation by regulating SHCBP1 in mice. Int Immunopharmacol. 2020;80:106123.

98. Huang J, Guo W, Cheung F, Tan HY, Wang N, Feng Y. Integrating network pharmacology and experimental models to investigate the efficacy of coptidis and Scutellaria containing Huanglian Jiedu decoction on hepatocellular carcinoma. Am J Chin Med. 2020;48:161-82.

99. Park HS, Park KI, Hong GE, Nagappan A, Lee HJ, Kim EH. Korean Scutellaria baicalensis Georgi methanol extracts inhibits metastasis via the Forkhead Box M1 activity in hepatocellular carcinoma cells. J Ethnopharmacol. 2014;155:847-51.

100. Han Z, Zhu S, Han X, Wang SW, Wang RZ. Baicalein inhibits hepatocellular carcinoma cells through suppressing the expression of CD24. Int Immunopharmacol. 2015;29:416-22.

101. Dong Q, Chu F, Wu C, Huo Q, Gan H, Li X. Scutellaria baicalensis Georgi extract protects against alcohol-induced acute liver injury in mice and affects the mechanism of ER stress. Mol Med Rep. 2016;13:3052-62.

102. Shi H, Zhang Y, Xing J, Liu L, Qiao F, Li J. Baicalin attenuates hepatic injury in non-alcoholic steatohepatitis cell model by suppressing inflammasome-dependent GSDMD-mediated cell pyroptosis. Int Immunopharmacol. 2020:81:106195.

103. Kim KT, Jeon GH, Cho SH, Lim SG, Kwon MG, Yoo JH. Effects of dietary inclusion of various concentrations of Scutellaria baicalensis Georgi extract on growth, body composition, serum chemistry and challenge test of far eastern catfish (silurus asotus). Aquac Res. 2013;44:1502-10.

104. Huang S, Huang Q, Huang B, Lu F. The effect of Scutellaria baicalensis Georgi on immune response in mouse model of experimental periodontitis. J Dent Sci. 2013;8:405-11.

105. Fu S, Zhao W, Xiong C, Guo L, Guo J, Qiu Y. Baicalin modulates apoptosis via RAGE, MAPK, and AP-1 in vascular endothelial cells during Haemophilus parasuis invasion. Innate Immun. 2019;25:420-32.

106. Zhao F, Chang Y, Gao L, Qin X, Du G, Zhang X. Protective effects of Scutellaria baicalensis Georgi extract on D-galactose induced aging rats. Metab Brain Dis. 2018;33:1401-12.

107. Song J, Zhou YZ, Pang YY, Gao L, Du GH, Qin XM. The anti-aging effect of Scutellaria baicalensis Georgi flowers extract by regulating the glutamine-glutamate metabolic pathway in D-galactose induced aging rats. Exp Gerontol. 2020;134:110843.

108. Lim H, Kwon YS, Kim DH, Lee JK, Kim HP. Flavonoids from Scutellaria baicalensis inhibit senescence-associated secretory phenotype production by interrupting $1 \mathrm{~KB} C / \mathrm{C} / \mathrm{EBP} \beta$ pathway: inhibition of age-related inflammation. Phytomedicine. 2020;76:153255.

109. Gu Y, Chen X, Wang Y, Liu Y, Zheng L, Li X. Development of 3-mercaptopropyltrimethoxysilane (MPTS)-modified bone marrow mononuclear cell membrane chromatography for screening anti-osteoporosis components from Scutellariae Radix. Acta Pharm Sin B. 2020.

110. Liu J, Wang S, Sun J, Shi J, Li Y, Gou J. Screening of osteoanagenesisactive compounds from Scutellaria baicalensis Georgi by hPDLC/CMConline-HPLC/MS. Fitoterapia. 2014;93:105-14.

111. Kim MH, Ryu SY, Bae M, Choi JS, Min YK. Baicalein inhibits osteoclast differentiation and induces mature osteoclast apoptosis. Food Chem Toxicol. 2008:46:3375-82.

112. Kim JM, Lee SU, Kim YS, Min YKKS. Baicalein stimulates osteoblast differentiation via coordinating activation of MAP kinases and transcription factors. J Cell Biochem. 2008;104:1906-17.

113. Jin BR, Chung KS, Kim HJ, An HJ. Chinese Skullcap (Scutellaria baicalensis Georgi) inhibits inflammation and proliferation on benign prostatic hyperplasia in rats. J Ethnopharmacol. 2019;235:481-8.
114. Fisar Z. Drugs related to monoamine oxidase activity. Prog Neuropsychopharmacol Biol Psychiatry. 2016;69:112-24.

115. Lee HW, Ryu HW, Kang MG, Park D, Lee H, Shin HM. Potent inhibition of monoamine oxidase A by decursin from Angelica gigas Nakai and by wogonin from Scutellaria baicalensis Georgi. Int J Biol Macromol. 2017;97:598-605.

116. Zhang RY, Ma ZX, Liu KL, Li YW, Liu DN, Xu LX, Deng XY, Qu R, Ma ZQ, Ma SP. Baicalin exerts antidepressant effects through Akt/FOXG1 pathway promoting neuronal differentiation and survival. Life Sci. 2019;221:241-8

117. Kudo M, Kobayashi-Nakamura K, Tsuji-Naito K. Bifunctional effects of O-methylated flavones from Scutellaria baicalensis Georgi on melanocytes: inhibition of melanin production and intracellular melanosome transport. PLoS ONE. 2017;12:1-26.

118. Trinh HT, Joh EH, Kwak HY, Baek NI, Kim DH. Anti-pruritic effect of baicalin and its metabolites, baicalein and oroxylin $\mathrm{A}$, in mice. Acta Pharmacol Sin. 2010;31:718-24.

119. Hou Y, Lin S, Tsai S, Ko M, Chang Y, Chao PL. Flavonoid pharmacokinetics and tissue distribution after repeated dosing of the roots of Scutellaria baicalensis in rats. Planta Med. 2011;77:455-60.

120. Xing S, Wang M, Peng Y, Chen D, Li X. Simulated gastrointestinal tract metabolism and pharmacological activities of water extract of Scutellaria baicalensis roots. J Ethnopharmacol. 2014;152:183-9.

121. Zhi HJ, Jin X, Zhu HY, Li H, Zhang YY, Lu Y. Exploring the effective materials of flavonoids-enriched extract from Scutellaria baicalensis roots based on the metabolic activation in influenza A virus induced acute lung injury. J Pharm Biomed Anal. 2020;177:112876.

122. Ren $\mathrm{G}$, Chen $\mathrm{H}$, Zhang M, Yang $\mathrm{N}$, Yang H, Xu C. Pharmacokinetics, tissue distribution and excretion study of Oroxylin A, Oroxylin A 7-O-glucuronide and Oroxylin A sodium sulfonate in rats after administration of Oroxylin A. Fitoterapia. 2020;142:104480.

123. Li M, Shi A, Pang H, Xue W, Li Y, Cao G. Safety, tolerability, and pharmacokinetics of a single ascending dose of baicalein chewable tablets in healthy subjects. J Ethnopharmacol. 2014;156:210-5.

124. Shii T, Kuroda M, Shamoto N, Mimaki Y. An analysis of the ingredients in decoctions and extracts of Kampo medicines: Amounts of baicalin and baicalein in Kampo medicines containing Scutellariae Radix. J Japanese Geriatr Soc. 2020;57:72-80.

125. Zhao L, Chen Z, Zhao Q, Wang D, Hu R, You Q. Developmental toxicity and genotoxicity studies of wogonin. Regul Toxicol Pharmacol. 2011;60:212-7.

126. Zhang W, Song DR, Wang YN. Evaluation of embryonic toxicity of baicalin based on embryonic stem cell experimental model. Chinese J Pharmacol Toxicol. 2012;16:864-9.

127. Deng YY, Hu ZM, Zhou Y, Chen SZ, Liu JP. Determination of specific antibody in serum of rabbits sensitized to baicalin in shuanghuanglian injection. Hebei JTCM. 2017;39:880-3.

128. Gao N, Gao Y, Tian FQ, Qiao HL. Sensitization of baicalin in guinea pigs and its possible mechanism. Chin J Pharmacol Toxicol. 2014;28:857-62.

129. Wang J, Zhang YJ, Che DL, Zeng YN, Wu YY, Qin QH, Wang N. Baicalin induces Mrgprb2-dependent pseudo-allergy in mice. Immunol Lett. 2020;226:55-61.

130. Cai Y, Ma W, Xiao Y, Wu B, Li X, Liu F. High doses of baicalin induces kidney injury and fibrosis through regulating TGF- $\beta /$ Smad signaling pathway. Toxicol Appl Pharmacol. 2017:333:1-9.

131. Adam T, Bursztejn AC, Schmutz JL. Facial eczema from a sunscreen: Scutellaria baicalensis, a novel allergen beginning to attract attention. Contact Dermatitis. 2020;82:253-4.

132. Gallo R, Pastorino C, Gasparini G, Ciccarese GPA. Scutellaria baicalensis extract: a novel botanical allergen in cosmetic products? Contact Dermatitis. 2016;75:387-8.

133. Scheers C, Dehavay F, Andre J, Neczyporenko FKA. A case of recalcitrant face eczema. Contact Dermatitis. 2019;80:242-3.

134. Hussain S, Xie Y, Li D, Malik SL, Hou JC, Leung LH, Fan XX. Current strategies against COVID-19. Chin Med. 2020;15:70.

135. Tian XL, Li C, Huang AL, Xia S, Lu SC, Shi ZL, Lu L, Jiang SB, Yang ZL, Wu YL. Potent binding of 2019 novel coronavirus spike protein by a SARS coronavirus-specific human monoclon alantibody. Emerg Microbes Infect. 2020;9:382-5.

136. Chen Y, Liu QY, Guo DY. Emerging coronaviruses: genome structure, replication, and pathogenesis. J Med Virol. 2020;92(4):418-23. 
137. Zhao Y, Zhao Z, Wang Y, Zhou Y, Ma Y, Zuo W. Single-cell RNA expression profiling of ACE2, the putative receptor of Wuhan 2019-nCov. bioRxiv. 2020.

138. Huang CL, Wang YM, Li XW, Ren LL, Zhao JP, Hu Y. Clinical features of patients infected with 2019 novel coronavirus in Wuhan, China. Lancet. 2020;S0140-6736(20):30183-5.

139. Chen F, Chan KH, Jiang Y, Kao RYT, Lu HT, Fan KW. In vitro susceptibility of 10 clinical isolates of SARS coronavirus to selected antiviral compounds. J Clin Virol. 2004:31:69-75.

140. Chen H, Du Q. Potential natural compounds for preventing 2019-nCoV infection. Chin Med. 2020

141. Ruan X, Du P, Zhao K, Huang JC, Xia HM, Dai D, Huang S, Cu X, Liu LM, Zhang JJ. Mechanism of Dayuanyin in the treatment of coronavirus disease 2019 based on network pharmacology and molecular docking. Chin Med. 2020;15:62.

142. Su HX, Yao S, Zhao WF, Li MJ, Liu J, Shang WJ, Xie H, Ke CQ. Discovery of baicalin and baicalein as novel, natural product inhibitors of SARSCoV-2 3CL protease in vitro. bioRxiv. 2020.04.13.038687.

143. Wenting WJ, Noormaimaiti MT, Wumaier AW, Yusufu MD, Noor MD, Mahemuti NZ. Study on the active components in the adjuvant treatment of novel coronavirus pneumonia (COVID-19) with Jinhua Qinggan granules based on network pharmacology and molecular docking. J Chinese Med Mater. 2020.
144. Wang Y, Wu J, Xiang JY, Li HM, Zhou S, Wang GQ, Shi YH, Bin W. Exploring the active compounds of Huanglian Jiedu decoction in the treatment of coronavirus disease 2019 (COVID-19) based on network pharmacology and molecular docking method. Pharmacol Clin Chinese Mater Med. 2020.

145. Tong T, Wu Y, Ni W, Shen AZ, Liu S. The potential insights of Traditional Chinese Medicine on treatment of COVID-19. Chin Med. 2020;15:51.

146. Jaffe S. Regulators split on antimalarials for COVID-19. Lancet. 2020;395:1179.

147. The $\mathrm{ClICl}$, press $\mathrm{SClOha}$, traditional cotiro, treatment Cmitpa, drugs. OC-AE. 2020

148. of XITd, the tCmaei, treatment of novel coronary pneumonia t, propose tmatd, Chinese a-ppot, Medicine. 2020.

149. Lane R. Sarah Gilbert: carving a path towards a COVID-19 vaccine. Lancet. 2020;395:1247.

150. Liu X, Zhang Y, Wu M, Ma MH, Huang ZH, Tian F, Dong SH. The scientific elucidation of daodi medicinal materials. Chin Med. 2020;15:86.

\section{Publisher's Note}

Springer Nature remains neutral with regard to jurisdictional claims in published maps and institutional affiliations.
Ready to submit your research? Choose BMC and benefit from:

- fast, convenient online submission

- thorough peer review by experienced researchers in your field

- rapid publication on acceptance

- support for research data, including large and complex data types

- gold Open Access which fosters wider collaboration and increased citations

- maximum visibility for your research: over $100 \mathrm{M}$ website views per year

At BMC, research is always in progress.

Learn more biomedcentral.com/submissions 